\title{
Variedades não matriciais em certas classes de álgebras não associativas
}

\author{
Vinicius Souza Bittencourt
}

TESE APRESENTADA

$\mathrm{AO}$

Instituto de MATEMÁticA E EstatísticA

DA

Universidade DE SÃo PaUlo

PARA

OBTENÇÃO DO TÍTULO

$\mathrm{DE}$

Doutor EM CiÊNCIAS

Programa: Matemática

Orientador: Prof. Dr. Ivan P. Shestakov

Durante o desenvolvimento deste trabalho o autor recebeu auxílio financeiro da CNPq

São Paulo, maio de 2016 


\section{Variedades não matriciais em certas classes de álgebras não associativas}

Esta versão da tese contém as correções e alterações sugeridas pela Comissão Julgadora durante a defesa da versão original do trabalho, realizada em 03/05/2016. Uma cópia da versão original está disponível no Instituto de Matemática e Estatística da Universidade de São Paulo.

Comissão Julgadora:

- Prof. Dr. Ivan Pavlovich Shestakov (orientador) - IME-USP

- Profa. Dra. Lucia Satie Ikemoto Murakami - IME-USP

- Prof. Dr. Alexandr Kornev - UFABC

- Prof. Dr. Victor Petrogradskiy - UnB

- Prof. Dr. Thierry Corrêa Petit Lobão - UFBA 


\section{Agradecimentos}

Agradeço ao meu orientador, Prof. Ivan Shestakov, pela paciência, atenção e dedicação empreendidas neste trabalho, além de contribuir grandemente para o aperfeiçoamento da minha formação matemática.

Agradeço à minha família, Lourival, Lícia e Viviane, pelo suporte, apoio e carinho.

Agradeço à minha querida Angela, um braço forte nesta reta final da tese.

Agraeço aos meus parentes, tão tão distantes geograficamente, pela torcida e pela força!

Agradeço ao corpo docente do IME pela dedicação nas disciplinas, palestras e cursos ministrados.

Agradeço ao professor Thierry Lobão, por haver me apresentado os "primeiros passos" da pesquisa matemática.

Agradeço aos meus amigos em São Paulo, em especial Iremar, Esdras, Lucas, Alex, Eduardo e Alessandra, pela força e pelas festas!

Agradeço aos meus amigos torcedores do Vitória (minha vida!) residentes em São Paulo, pelos momentos de alegria.

Agradeço às famílias Oliveira e Melo, por partilharem um pouco desse processo comigo. Agradeço aos meus queridos ex-alunos e colegas da EBEI.

Agradeço ao meu amigo Luiz Marcio, por apresentar-me o lado negro da Força.

Agradeço aos amigos da minha terra natal, por torcerem tanto, ainda que à distância. 


\section{Resumo}

BITTENCOURT, V. S. Variedades não matriciais em certas classes de álgebras não associativas. 2016. 81 f. Tese (Doutorado) - Instituto de Matemática e Estatística, Universidade de São Paulo, São Paulo, 2016.

Uma variedade $\mathcal{M}$ de álgebras associativas é dita ser "não matricial" se $F_{2} \notin \mathcal{M}$, em que $F_{2}$ é o anel das matrizes quadradas de ordem 2 sobre $F$. V. Latyshev introduziu estas variedades em [Lat77]. A respeito desta definição, outras caracterizações equivalentes para uma variedade não matricial foram obtidas, por exemplo, ao considerar elementos algébricos [Cek79] e nilpotentes [MPR2011]. Variedades não matriciais são estudadas principalmente no caso sobre os corpos de característica zero para álgebras associativas.

A teoria geral de variedades de álgebras, entretanto, não está restrita à classe das álgebras associativas. Além das álgebras de Lie, entre as muitas classes de álgebras não associativas, nós destacamos as álgebras alternativas, as de Jordan e as de Jordan não comutativas. Estas classes de álgebras têm muitas conexões e aplicações a diversas áreas da Matemática e da Física e têm uma teoria estrutural bem desenvolvida, assim como a classe das álgebras associativas.

O conceito de "variedade não matricial" pode ser reformulado para as classes de álgebras supracitadas e nosso trabalho consiste em adaptar, estender ou generalizar alguns resultados, conforme mencionado, para variedades não matriciais nestas classes de álgebras.

Palavras-chave: álgebra, não matricial, variedade, PI, não associativo. 


\section{Abstract}

BITTENCOURT, V. S. Nonmatrix varieties in certain classes of non associative algebras. 2016. 81 s. Tese (Doutorado) - Instituto de Matemática e Estatística, Universidade de São Paulo, São Paulo, 2016.

A variety $\mathcal{M}$ of associative algebras (over a field $F$ ) is called "nonmatrix" if $F_{2} \notin M$, where $F_{2}$ is the usual matrix algebra of second order over $F$. V. Latyshev introduced these varieties in [Lat77]. Concerning this definition, other equivalent characterizations for a nonmatrix variety were obtained, for instance, by considering algebraic [Cek79] and nilpotent [MPR2011] elements. Non-matrix varieties are studied mainly in the case of characteristic zero for associative algebras.

However, the general theory of varieties of algebras is not restricted to the class of associative algebras. In addition to the Lie algebras, among many classes of non associative algebras, we highlight the alternative, the Jordan and the non commutative Jordan algebras. These classes of algebras have many connexions and applications to several areas of Mathematics and Physics and have a well-developed structural theory, as in the class of associative algebras.

The concept of "nonmatrix variety" can be reformulated in the classes of algebras above and our work is to adapt, extend or generalize some results, as mentioned, for non-matrix varieties in these classes of algebras.

Keywords: algebra, nonmatrix, variety, PI, non associative. 


\section{Sumário}

Lista de Símbolos $\quad$ ix

1 Introdução 1

1.1 Considerações Preliminares . . . . . . . . . . . . . . . . . . . . 1

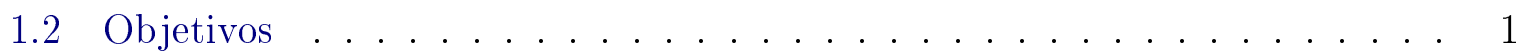

1.3 Contribuições . . . . . . . . . . . . . . . . . . . 2

1.4 Organização do Trabalho . . . . . . . . . . . . . . . . . 2

2 Elementos gerais em álgebras $\quad 3$

2.1 Definições básicas . . . . . . . . . . . . . . . . . . . . . . . 3

2.2 Variedades . . . . . . . . . . . . . . . . . . . 9

2.3 Elementos da Teoria Geral de Radicais . . . . . . . . . . . . . . . . . 11

2.3.1 Exemplos de radicais . . . . . . . . . . . . . . 15

2.4 PI-álgebras associativas . . . . . . . . . . . . . . . . 22

2.5 Variedades não matriciais em Assoc . . . . . . . . . . . . . . . 24

3 Álgebras de Jordan $\quad 27$

3.1 Definições e exemplos . . . . . . . . . . . . . . . . . . . . . 29

$3.1 .1 \quad$ PI-álgebras de Jordan . . . . . . . . . . . . . . . 30

3.2 Radicais em Jord . . . . . . . . . . . . . . . . . . . . . . . 31

3.3 Variedades não matriciais em Jord . . . . . . . . . . . . . . . . 36

4 Âlgebras alternativas $\quad 45$

4.1 Definições e exemplos . . . . . . . . . . . . . . . . . . . . . 45

4.2 Radicais em Alt . . . . . . . . . . . . . . . . . 46

4.3 Variedades não matriciais em Alt . . . . . . . . . . . . . . 48

4.4 O caso não matricial para $(-1,1)$-álgebras . . . . . . . . . . . . . . 51

5 Âlgebras de Jordan não-comutativas $\quad 57$

5.1 Exemplos de álgebras em NCJ . . . . . . . . . . . . . 58

5.1 .1 Álgebras de Jordan não comutativas estritamente primas . . . . . 59 
viii SUMÁRIO

5.2 Álgebras de Jordan não comutativas admissíveis . . . . . . . . . . . . . . 62

5.3 Variedades não matriciais em NCJ . . . . . . . . . . . . . . 67

6 Conclusões $\quad 75$

6.1 Sugestões para Pesquisas Futuras . . . . . . . . . . . . . . 75

6.2 Considerações Finais . . . . . . . . . . . . . . . . . . 77

$\begin{array}{ll}\text { Referências Bibliográficas } & 79\end{array}$ 


\title{
Lista de Símbolos
}

$(x, y, z)$
$[x, y]$
$x \circ y$
$A^{(+)}$
$(x, y, z)_{+}$
$\mathcal{V}, \mathcal{M}$
Assoc
Com
Jord
Alt
NCJ
$F$
$\Phi$
$\mathbb{N}$
$\mathbb{Z}$
$\mathbb{Q}$
$\mathbb{R}$
$\mathbb{C}$
$\mathbb{H}$
$\mathbb{O}$

\author{
associador: $(x y) z-x(y z)$ \\ comutador: $x y-y x$ \\ multiplicação de Jordan: $\frac{1}{2}(x y+y x)$ \\ $(\mathrm{A},+, \circ)$ \\ associador em $\mathbf{A}^{(+)}$ \\ variedades de álgebras \\ variedade das álgebras associativas \\ variedade das álgebras comutativas \\ variedade das álgebras de Jordan \\ variedade das álgebras alternativas \\ variedade das álgebras de Jordan não comutativas \\ corpo (geralmente infinito) \\ anel de escalares para álgebras \\ conjunto dos números naturais \\ anel dos inteiros \\ corpo dos racionais \\ corpo dos reais \\ corpo dos complexos (usuais) \\ anel de divisão dos quatérnios (usuais) \\ álgebra dos octônios (usuais)
}




\section{Capítulo 1}

\section{Introdução}

Uma variedade $\mathcal{M}$ de álgebras associativas é dita ser "não matricial" se $F_{2} \notin \mathcal{M}$, em que $F_{2}$ é o anel das matrizes quadradas de ordem 2 sobre $F$. Tais variedades foram introduzidas por Latyshev em 1977, [Lat77].

A teoria de variedades de álgebras não está restrita à classe das álgebras associativas. Dentre as muitas classes de álgebras não associativas, nós destacamos as álgebras alternativas, as álgebras de Jordan e as álgebras de Jordan não comutativas. Estas classes de álgebras têm muitas conexões e aplicações a diversas áreas da Matemática e da Física e têm uma teoria estrutural bem desenvolvida, assim como a classe das álgebras associativas.

O conceito de "variedade não matricial" pode ser reformulado para as classes de álgebras supracitadas e nosso trabalho consiste em adaptar, estender ou generalizar alguns resultados, conforme mencionado, para variedades não matriciais nestas classes de álgebras.

\subsection{Considerações Preliminares}

Ao longo deste trabalho, uma álgebra será uma $\Phi$-álgebra, em que $\Phi$ é um anel de escalares, isto é, $\Phi$ é um anel associativo, comutativo e com elemento identidade.

A letra $F$ será usada para denotar corpos (quase sempre, corpos infinitos). Notaremos as matrizes $2 \times 2$ sobre $F$ por $F_{2}$ e as matrizes hermitianas sobre $F_{2}$ por $\mathcal{H}_{2}$.

\subsection{Objetivos}

Nosso principal objetivo é oferecer uma caracterização não matricial para variedades em algumas classes de álgebras não associativas. Como há muitas classes de álgebras não associativas conhecidas e estudadas na literatura, nos ateremos a quatro classes deste tipo: a classe das álgebras de Jordan, a classe das álgebras alternativas, a classe das álgebras $(-1,1)$ e a classe das álgebras de Jordan não comutativas.

Para tanto, desenvolveremos novas construções e novas técnicas concernentes para álgebras não associativas. 


\subsection{Contribuições}

As principais contribuições deste trabalho são as seguintes:

- Uma nova maneira de conceituar variedades não matriciais;

- A generalização de técnicas envolvendo conceitos de "não matricialidade" para álgebras não associativas.

Julgamos tais contribuições como relevantes para este trabalho, bem como para a literatura matemática.

\subsection{Organização do Trabalho}

No Capítulo 2, apresentamos conceitos básicos relativos a este trabalho. Indicamos uma Bibliografia Básica para um leitor não muito familiarizado com o assunto, mas também para um leitor mais experiente e que necessita ter uma referência. Apresentamos os objetos matemáticos que servirão de apoio a esta tese.

No Capítulo 3, apresentamos a definição de variedade não matricial em álgebras de Jordan. De posse desta definição, provamos que, para álgebras de Jordan, vale o análogo de uma caracterização não matricial feita para as classes das álgebras associativas. Ainda, apresentamos uma nova maneira de conceituar variedades não matriciais, pois tentaremos universalizar o conceito de "não matricial" para qualquer classe de álgebras.

No Capítulo 4, apresentamos uma extensão (com respeito às álgebras associativas) da caracterização de variedade não matricial para as álgebras alternativas. Além disso, exibimos uma caracterização não matricial para uma classe de álgebras alternativas à direita, as álgebras $(-1,1)$.

No Capítulo 5, apresentamos uma série de novos conceitos relativos às álgebras de Jordan não comutativas, com o objetivo de fornecer uma caracterização não matricial. De fato, fomos capazes de caracterizar variedades não matriciais em uma classe particular, a classe das álgebras de Jordan não comutativas admissiveis, que é uma classe que contém as álgebras associativas e as álgebras comutativas.

Finalmente, no Capítulo 6, elaboramos a conclusão deste trabalho, discutindo os resultados e as técnicas utilizadas, além de apresentar questões e problemas em aberto relativos ao temas aqui abordados. 


\section{Capítulo 2}

\section{Elementos gerais em álgebras}

Neste capítulo, serão listados alguns resultados básicos de álgebras. Para um leitor não familiarizado com o assunto, recomendamos a leitura de [Hung74], [Lang02], [G69] e [ZSSS82]. Doravante, essas citações serão chamadas de Bibliografia Básica.

\subsection{Definições básicas}

Definição 2.1.1 ( $\Phi$-álgebra). Seja $\Phi$ um anel associativo, comutativo e unitário. Diremos que um $\Phi$-módulo unitário A é uma álgebra se houver uma multiplicação "." definida sobre $A$ que satisfaça os critérios abaixo:

A1) $(a+b) c=a c+b c$, para quaisquer $a, b, c \in A$;

A2) $c(a+b)=c a+c b$, para quaisquer $a, b, c \in A$;

A1) $\gamma(a b)=(\gamma a) b=a(\gamma b)$, para quaisquer $a, b \in A$ e $\gamma \in \Phi$.

A menos que se especifique o contrário, $\Phi$ sempre denotará um anel associativo, comutativo e unitário, conhecido também como anel de operadores da álgebra $\mathbf{A}$.

Definição 2.1.2 (Classe de equivalência). Sejam $I$ um ideal de um anel $R$ e $x, y$ elementos de $R$. Dizemos que $x$ é equivalente a $y$ (módulo $I$ ) se, e somente se, $x-y \in I$. Notação: $x \equiv y$. Isso define uma relação de equivalência em $R$ e denotaremos a classe de equivalência a qual $x$ faz parte por

$$
x+I=\bar{x}=\{x+a ; a \in I\} .
$$

Podemos definir operações (induzidas) no conjunto das classes a partir das operações do anel $R$, como a seguir:

1. $(x+I)+(y+I)=(x+y)+I$

2. $(x+I)(y+I)=x y+I$

O conjunto de todas as classes supracitadas munido das operações acima definidas será chamado de anel quociente módulo I e será denotado por $R / I$.

Definição 2.1.3 (Produto de ideais). Se $I$ e $J$ são ideais de uma álgebra A, então definimos o produto $I J$ entre os ideais da seguinte forma

$$
I J=\left\{\sum_{k} a_{k} b_{k} ; a_{k} \in I, b_{k} \in J\right\} .
$$


Seja $R$ um anel e consideremos o conjunto $M_{n}(R)$ como a seguir:

$$
M_{n}(R)=\left\{\left(\begin{array}{cccc}
a_{11} & a_{12} & \cdots & a_{1 n} \\
a_{21} & a_{22} & \cdots & a_{2 n} \\
\vdots & \vdots & \ddots & \vdots \\
a_{n 1} & a_{n 2} & \cdots & a_{n n}
\end{array}\right) ; a_{i j} \in A, 1 \leq i \leq n, 1 \leq j \leq n\right\} .
$$

O conjunto $M_{n}(R)$ munidos das operações "+" e "." usuais para matrizes é um anel, conhecido por anel de matrizes quadradas de ordem $n$ sobre o anel $R$ ou, simplesmente, anel de matrizes de ordem $n$ sobre o anel $R$.

Exemplo 2.1.4 (Anéis). Seja $R$ um anel e, dados um inteiro $k \in \mathbb{Z}$ e um elemento $a \in R$, defina que sobre $R$ a seguinte operação.

$$
k a=\underbrace{a+\cdots+a}_{k \text {-vezes }} .
$$

Com esta definição, adotaremos também as seguintes convenções:

$$
\underbrace{0}_{\in \mathbb{Z}} a=\underbrace{0}_{\in R}, \underbrace{1}_{\in \mathbb{Z}} a=a \text { e } \underbrace{(-1)}_{\in \mathbb{Z}} a=\underbrace{-a}_{\in R} .
$$

Desta forma, qualquer anel $R$ é uma $\mathbb{Z}$-álgebra.

Exemplo 2.1.5 (Zero álgebras). Uma $\Phi$-álgebra A é uma zero álgebra se $a b=0$, quaisquer que sejam $a, b \in \mathbf{A}$. Em outras palavras, uma zero álgebra é uma álgebra munida da multiplicação trivial.

Exemplo 2.1.6 (Álgebra de Grassmann). Sejam $K$ um corpo e $G$ um $K$-espaço vetorial. Defina em $G$ uma multiplicação "." associativa, que admite elemento identidade 1 e que, para os elementos de uma base $\left\{u_{\lambda}\right\}_{\lambda \in \Lambda}$ (base de $G$ como $K$-espaço vetorial), $u_{\lambda} \neq 1$, as seguintes relações sejam válidas:

$$
\begin{gathered}
u_{\lambda}^{2}=0 \\
u_{\lambda_{i}} u_{\lambda_{j}}=-u_{\lambda_{j}} u_{\lambda_{i}}, \text { se } \lambda_{i} \neq \lambda_{j} .
\end{gathered}
$$

Definimos a álgebra de Grassmann, denotada por $G$, como sendo a álgebra gerada pelos elementos $\{1\} \cup\left\{u_{\lambda}, \lambda \in \Lambda\right\}$, isto é,

$$
G=\operatorname{alg}\left\langle 1,\left\{u_{\lambda}\right\}_{\lambda \in \Lambda}, \quad u_{\lambda}^{2}=0, \quad u_{\lambda_{i}} u_{\lambda_{j}}=-u_{\lambda_{j}} u_{\lambda_{i}} \text {, se } \lambda_{i} \neq \lambda_{j}\right\rangle .
$$

Se $\Lambda=\{1, \cdots, n\}$, denotaremos tal álgebra de Grassmann por $G_{n}$.

Exemplo 2.1.7 (Álgebras comutativas). Uma álgebra A é dita ser comutativa se, para quaisquer elementos $a, b \in \mathbf{A}$, é equação abaixo é verdadeira:

$$
a b-b a=0 .
$$

Exemplo 2.1.8 (Álgebras anticomutativas). Uma álgebra A é dita ser anticomutativa se, para quaisquer elementos $a, b \in \mathbf{A}$, é equação abaixo é verdadeira:

$$
a b+b a=0 .
$$


Exemplo 2.1.9 (Álgebras associativas). Uma álgebra A é dita ser associativa se, para quaisquer elementos $a, b, c \in A$, é equação abaixo é verdadeira:

$$
(a b) c-a(b c)=0
$$

Exemplo 2.1.10 (Álgebras de potências associativas). Uma álgebra é dita ser de potências associativas se cada elemento gera uma subálgebra associativa.

Em outras palavras, se A é de potências associativas e $a \in \mathbf{A}$ é um elemento arbitrário, então qualquer arranjo multiplicativo com repetições do elementos $a$ não depende das possíveis alocações entre os parênteses.

Álgebras associativas são, evidentemente, álgebras de potências associativas. Todavia, alguns exemplos não triviais de álgebras de potências associativas serão considerados a seguir. O "não trivial" aí deve ser entendido da seguinte forma: álgebras que não são (necessariamente) associativas, mas são de potências associativas.

Exemplo 2.1.11 (Álgebras de Jordan). Uma álgebra A é dita ser de Jordan se, para quaisquer elementos $a, b \in \mathbf{A}$, as duas relações abaixo são válidas:

$$
\begin{aligned}
a b-b a & =0 \\
a^{2}(b a)-\left(a^{2} b\right) a & =0 .
\end{aligned}
$$

Note que as relações 2.1 e 2.4 coincidem, ou seja, álgebras de Jordan são álgebras comutativas que satisfazem 2.5.

Exemplo 2.1.12 (Álgebras alternativas). Uma álgebra A é dita ser alternativa se, para quaisquer elementos $a, b \in \mathbf{A}$, as duas relações abaixo são válidas:

$$
\begin{aligned}
(a b) b-a b^{2} & =0 \\
a^{2} b-a(a b) & =0 .
\end{aligned}
$$

A relação 2.6 é conhecida como alternatividade à direita. Por sua vez, a relação 2.7 é conhecida como alternatividade à esquerda. Portanto, álgebras alternativas são álgebras alternativas à direita e à esquerda.

Exemplo 2.1.13 (Álgebras flexíveis). Uma álgebra A é dita ser flexível se, para quaisquer elementos $a, b \in \mathbf{A}$, é equação abaixo é verdadeira:

$$
a(b a)-(a b) a=0 .
$$

Seja A uma álgebra comutativa (Exemplo 2.1.7) e sejam $a$ e $b$ elementos arbitrários de A. Observe que

$$
(a b) a-a(b a) \underbrace{=}_{\text {comutatividade }}(a b) a-(b a) a \underbrace{=}_{\text {comutatividade }}(a b) a-(a b) a=0 .
$$

As equações acima mostram que álgebras comutativas são flexíveis. 
Veremos, no Capítulo 5 (sobre álgebras de Jordan não comutativas), que a flexibilidade pode ser entendida como uma condição mais fraca à comutatividade.

Exemplo 2.1.14 (Álgebras de Jordan não comutativas). Uma álgebra A é dita ser de Jordan não comutativa se, para quaisquer elementos $a, b \in \mathbf{A}$, as duas relações abaixo são válidas:

$$
\begin{aligned}
& a(b a)-(a b) a=0 . \\
& \quad a^{2}(b a)-\left(a^{2} b\right) a=0 .
\end{aligned}
$$

A relação 2.9 mostra-nos que álgebras de Jordan não comutativas são álgebras flexíveis que satisfazem a identidade de Jordan 2.5.

Em [ZSSS82], pode-se verificar que álgebras de Jordan, álgebras de Jordan não comutativas e álgebras alternativas são álgebras de potências associativas. Essa informação nos será útil mais adiante.

Dados um conjunto $X$ munido de uma operação "." e fixado um elemento $a \in X$, definiremos as funções multiplicação à direita, denotada por $R_{a}$, e multiplicação à esquerda, denotada por $L_{a}$, da seguinte forma:

$$
\begin{aligned}
R_{a}: X & \rightarrow X \\
x & \mapsto x \cdot a ; \\
L_{a}: X & \rightarrow X \\
x & \mapsto a \cdot x .
\end{aligned}
$$

Adotaremos a notação $x R_{a}=x a$ para indicar a imagem do elemento através da aplicação $R_{a}$. Algumas obras usam a notação usual para funções, $R_{a}(x)=x a$, mas isso será evitado neste trabalho, apenas por convenção da literatura atual. O mesmo se aplica a $L_{a}$.

Os exemplos anteriores já sugerem que, nesse trabalho, nem sempre as operações serão associativas ou comutativas. Dessa forma, introduziremos dois operadores: o associador e o comutador.

Definição 2.1.15 (Associador). Sejam $X$ um conjunto e · uma operação sobre $X$. Dados $x, y$ e $z$ elementos arbitrários em $X$, definiremos o associador pela seguinte expressão:

$$
(x, y, z)=(x \cdot y) \cdot z-x \cdot(y \cdot z) .
$$

Definição 2.1.16 (Comutador). Sejam $X$ um conjunto e · uma operação sobre $X$. Dados $x$ e $y$ elementos arbitrários em $X$, definiremos o comutador através da expressão abaixo:

$$
[x, y]=x \cdot y-y \cdot x .
$$

Seja A uma $\Phi$-álgebra, em que $\Phi$ é um anel de operadores (que é unitário) tal que $1+1$ é um elemento inversível, isto é, $1 / 2 \in \Phi$. Podemos construir uma álgebra comutativa, a 
partir da multiplicação já definida em A, como a seguir:

$$
a \circ b=\frac{1}{2}(a b+b a)
$$

Segue imediatamente da definição de "o" que $a \circ b=b \circ a$, ou seja, $(A,+, \circ)$ é uma álgebra comutativa. Definimos, portanto, a álgebra adjunta $\mathbf{A}^{(+)}$como sendo $\mathbf{A}^{(+)}=(A,+, \circ) \mathrm{e}$ a multiplicação "o" também é conhecida como multiplicação de Jordan.

Em $\mathbf{A}^{(+)}$podemos também definir um associador relacionado à multiplicação de Jordan, a que chamaremos de associador + e denotaremos por $(a, b, c)_{+}$:

$$
(a, b, c)_{+}:=(a \circ b) \circ c-a \circ(b \circ c), \quad \forall a, b, c \in \mathbf{A} .
$$

Em [ZSSS82, p.53] encontramos o seguinte resultado:

Lema 2.1.17. Para quaisquer elementos $a, b, c \in \mathbf{A}$, a seguinte relação é válida:

$$
4(a, b, c)_{+}=(a, b, c)-(c, b, a)+(b, a, c)-(c, a, b)+(a, c, b)-(b, c, a)+[b,[a, c]],
$$

em que $(a, b, c)_{+}$é o associador definido em $\mathbf{A}^{(+)}=(\mathbf{A},+, \circ)$.

Seja A uma álgebra. Considere em A os seguintes subconjuntos:

$$
\begin{aligned}
N(\mathbf{A}) & =\{n \in \mathbf{A} \mid(n, A, A)=(A, n, A)=(A, A, n)=0\} \\
K(\mathbf{A}) & =\{k \in \mathbf{A} \mid[k, A]=0\} ; \\
Z(\mathbf{A}) & =N(\mathbf{A}) \cap K(\mathbf{A}) .
\end{aligned}
$$

Os conjuntos $N(\mathbf{A}), K(\mathbf{A})$ e $Z(\mathbf{A})$ são conhecidos, respectivamente, como centro associativo, centro comutativo e centro da álgebra $\mathbf{A}$ e são os principais subconjuntos centrais de uma álgebra $\mathbf{A}$.

Sejam A uma álgebra e $Z=Z(\mathbf{A})$ seu centro. De forma análoga à construção do corpo dos racionais $\mathbb{Q}$ a partir de $\mathbb{Z} \times \mathbb{Z}^{*}$, defina o sistema multiplicativo $Z^{-1}=Z \backslash\{0\}$ e considere a álgebra $Z^{-1} \mathbf{A}$. Se $B \cong Z^{-1} \mathbf{A}$, então diremos que $B$ é o fecho central de $\mathbf{A}$ ou A é uma ordem central em $B$.

Se $F$ é um corpo e A é uma $F$-álgebra tal que $Z(\mathbf{A})=Z=F$, então $\mathbf{A}$ é dita ser central sobre $F$. Um álgebra $\mathbf{A}$ é dita ser central simples se ela é central e simples (a simplicidade de uma álgebra segue-se no sentido usual da palavra: uma álgebra $B \neq(0)$ é simples se os únicos ideais bilaterais de $B$ são (0) e $B$ ). Se uma álgebra A é central simples com elemento identidade 1, então $Z(\mathbf{A})$ é isomorfo ao corpo $F$ através da multiplicação por escalar: $Z(\mathbf{A}) \cong F \cdot 1 \cong F$.

Definição 2.1.1 (Soma direta). Seja A uma álgebra e sejam $I_{1}, I_{2}, \ldots, I_{n}$ ideais de A. Se $\mathbf{A}=\sum_{j=1}^{n} I_{j}$ e $I_{j} \cap\left(\sum_{1 \leq l \leq n ; l \neq j} I_{l}\right)=(0)$, então diremos que A é uma soma direta dos ideais $I_{1}, I_{2}, \ldots, I_{n}$. Notação: $\mathbf{A}=I_{1} \oplus I_{2} \oplus \cdots I_{n}=\bigoplus_{j=1}^{n} I_{j}$.

A intenção agora é generalizar a noção de soma direta, trabalhando com a noção de soma para uma família de álgebras que pode ser infinita. Seja $\left\{\mathbf{A}_{\lambda}\right\}_{\lambda \in \Lambda}$ uma coleção 
de álgebras indexadas por um conjunto $\Lambda$ (de cardinalidade arbitrária). Consideremos o conjunto $S=\prod_{\lambda \in \Lambda} \mathbf{A}_{\lambda}$ ("П" denota o produto cartesiano) munido das operações adição e multiplicação definidas coordenada a coordenada, isto é,

$$
\left(a_{\lambda}\right)_{\lambda}+\left(b_{\lambda}\right)_{\lambda}=\left(a_{\lambda}+b_{\lambda}\right)_{\lambda}
$$

$\mathrm{e}$

$$
\left(a_{\lambda}\right)_{\lambda} \cdot\left(b_{\lambda}\right)_{\lambda}=\left(a_{\lambda} \cdot b_{\lambda}\right)_{\lambda}
$$

Definido dessa forma (as operações em questão estão bem definidas), $S$ é dito ser o produto direto das álgebras $A_{\lambda}, \lambda \in \Lambda$, e pode ser considerada como uma extensão da soma direta pois o conjunto dos elementos da forma

$$
\left\{\begin{array}{rl}
a_{\alpha}, & \text { se } \lambda=\alpha \\
0, & \text { se } \lambda \neq \alpha
\end{array}:=\left(0, \cdots, 0, a_{\alpha}, 0, \cdots, 0\right), \text { na } \alpha\right. \text {-ésima posição, }
$$

é um ideal $\mathbf{A}_{\alpha}^{\prime}$ de $S$ que é isomorfo a $\mathbf{A}_{\alpha}$ e a aplicação

$$
\begin{array}{llc}
S=\prod_{\left(a_{\lambda}\right)_{\lambda}} \mathbf{A}_{\lambda} & \rightarrow & \mathbf{A}_{\alpha}^{\prime}
\end{array} \quad \text { ( } \alpha \text {-ésima posição) }
$$

é um epimorfismo de $S$ em $\mathbf{A}_{\alpha}^{\prime}$.

A álgebra $S_{w}$ de $S$ que consiste de todos os elementos que possuem uma quantidade finita de entradas não nulas é chamado de soma direta da coleção $\left\{A_{\lambda}\right\}_{\lambda \in \Lambda}$. Note que o homomorfismo natural definido acima, quando restrito a $S_{w}$, é uma sobrejeção de $S_{w}$ em $A_{\alpha}^{\prime}$, qualquer que seja $\alpha \in \Lambda$. Diremos que a subálgebra $S^{*}$ de $S$ é uma soma subdireta da coleção $\left\{A_{\lambda}\right\}_{\lambda \in \Lambda}$ se o homomorfismo natural de $S^{*}$ em $A_{\alpha}^{\prime}$,

$$
\left(a_{\lambda}\right)_{\lambda} \mapsto\left(0 \cdots 0, a_{\alpha}, 0 \cdots 0\right)
$$

é sobrejetivo, para todo $\alpha \in \Lambda$. Em particular, estamos dizendo que toda soma direta completa é uma soma subdireta.

Se a álgebra A é uma soma subdireta da coleção $\left\{\mathbf{A}_{\lambda}\right\}_{\lambda \in \Lambda}$, então usaremos o símbolo “乌” para expressar essa condição:

$$
\mathbf{A} \hookrightarrow \prod_{\lambda \in \Lambda} \mathbf{A}_{\lambda}
$$

Aproveitando este espaço, que trata de produtos cartesianos, é útil relembrarmos a definição de tensor. Sejam $R$ um anel, $A$ um $R$-módulo à direita, $B$ um $R$-módulo à esquerda e $G$ um grupo abeliano, definimos o produto balanceado $P: A \times B \rightarrow G$ como sendo uma aplicação bilinear tal que

$$
P(a \cdot r, b)=P(a, r \cdot b), \quad \forall a \in A, \forall b \in B, \forall r \in R .
$$

O produto tensorial $A \otimes_{R} B$ é definido (unicamente) via propriedade universal (para o produto balanceado $P$ ) através da aplicação

$$
\otimes: \quad A \times B \rightarrow A \otimes_{R} B .
$$

Consulte mais detalhes na Bibliografia Básica. 
Nesta seção, foram expostas definições e resultados gerais acerca da estrutura das álgebras. A seguir, nos ocuparemos em fundamentar objetos que têm maior significância para nosso estudo.

\section{$2.2 \quad$ Variedades}

Variedades de álgebras são classes de álgebras determinadas por certas identidades.

Fixe um conjunto arbitrário $X=\left\{x_{\alpha}\right\}$ e adicione a ele mais dois símbolos, parênteses à esquerda "(" e parênteses à direita ")", para obter um novo conjunto $X^{*}=X \cup\{(,)$,$\} .$ Considere todas as possíveis sequências finitas de elementos do conjunto $X^{*}$ que não possuam trivialidades ou posições inadequadas para os parênteses (por exemplo, despreze as palavras "()", ")x(", "y(xz(", etc). Duas sequências finitas $a_{1} a_{2} \cdots a_{m}$ e $b_{1} b_{2}, \cdots b_{n}$, em que $a_{i}, b_{j} \in X^{*}$, são consideradas iguais se $m=n$ e $a_{i}=b_{i}$, para $i=1,2, \cdots, m$. Definimos indutivamente um conjunto $V[X]$ dessas sequências de elementos do conjunto $X^{*}$, o qual chamaremos de palavras não associativas do conjunto $X$. Primeiro, todos os elementos do conjunto $X$ pertencem ao conjunto $V[X]$. Segundo, se $x_{1}, x_{2} \in X$ e $u, v \in V[X] \backslash X$, então as seqüências $x_{1} x_{2}, x_{1}(u),(v) x_{2}$ e $(u)(v)$ também pertencem ao conjunto $V[X]$. Nem toda sequência de elementos de $X^{*}$ é um elemento de $V[X]$. Por exemplo, a sequência $\left(x_{1}\left(x_{2} x_{3}\right)\right) x_{4}$ é uma palavra não associativa do conjunto $X=\left\{x_{1}, x_{2}, x_{3}, x_{4}\right\}$, mas a sequência $\left(x_{1}\left(x_{2} x_{3}\right) x_{4}\right)$ não é. $\mathrm{O}$ número de elementos do conjunto $X$ que aparecem em uma palavra $u$ é chamado de comprimento da palavra não associativa $u$ e é indicado por $d(u)$.

Podemos definir sobre $V[X]$ uma multiplicação. Sejam $x_{1}, x_{2} \in X$ e $u, v \in V[X] \backslash X$. Defina:

$$
\begin{aligned}
& x_{1} \cdot x_{2}=x_{1} x_{2} ; \\
& x_{1} \cdot u=x_{1}(u) ; \\
& v \cdot x_{2}=(v) x_{2} ; \\
& u \cdot v=(u)(v) .
\end{aligned}
$$

Considere agora o $\Phi$-módulo unitário e livre, $\Phi[X]$, sobre o conjunto $V[X]$ e extenda a operação de multiplicação definida em $V[X]$ para $\Phi[X]$ pela regra

$$
\left(\sum_{i} \alpha_{i} u_{i}\right) \cdot\left(\sum_{j} \beta_{j} u_{j}\right)=\sum_{i, j} \alpha_{i} \beta_{j}\left(u_{i} \cdot u_{j}\right),
$$

em que $\alpha_{i}, \beta_{j} \in \Phi$ e $u_{i}, v_{j} \in V[X]$. Com essa construção, obtemos a álgebra $\Phi[X]$, que é chamada de álgebra livre sobre o anel de $\Phi$ do conjunto de geradores $X$.

Fixe, agora, um conjunto enumerável de símbolos $X=\left\{x_{1}, x_{2}, \cdots\right\}$. Seja $f$ um elemento arbitrário de $\Phi[X]$. Logo, deve aparecer em seu suporte apenas um número finito de elementos de $X$, por exemplo $x_{1}, x_{2}, \cdots, x_{n}$. Neste caso, vamos escrever $f=$ $f\left(x_{1}, x_{2}, \cdots, x_{n}\right)$. Se $A$ é uma $\Phi$-álgebra e $a_{1}, \ldots, a_{n}$ são elementos arbitrários em $A$, então é possível mostrar que existe um único homomorfismo $\theta: \Phi[X] \rightarrow A$, tal que $x_{i} \mapsto a_{i}$, para $i=1, \cdots, n$, e aplica os outros elementos de $X$ no zero. Iremos denotar a imagem do elemento $f$ pelo homomorfismo $\theta$ por $f\left(a_{1}, a_{2}, \cdots, a_{n}\right)$ e dizer que o elemento $f\left(a_{1}, a_{2}, \ldots, a_{n}\right)$ é obtido por substituição do elementos $a_{1}, a_{2}, \cdots, a_{n}$ no polinômio não associativo $f\left(x_{1}, x_{2}, \cdots, x_{n}\right)$. 
Definição 2.2.1 (Identidade). Um polinômio não associativo $f=f\left(x_{1}, x_{2}, \cdots, x_{n}\right) \in$ $\Phi[X]$ é chamado de identidade da álgebra $A$, se $f\left(a_{1}, a_{2}, \cdots, a_{n}\right)=0$ para quaisquer $a_{1}, a_{2}, \cdots, a_{n} \in A$. Dizemos também que $A$ satisfaz a identidade $f$ ou que a identidade $f$ é válida em $A$.

A coleção de todas as identidades de uma certa álgebra é um ideal da álgebra $\Phi[X]$, a qual é chamada ideal das identidades ( $T$-ideal) da álgebra de A e é denotado por $T(A)$. A coleção de todas as identidades que são satisfeitas por cada álgebra de uma certa classe $\mathcal{M}$ também é um ideal em $\Phi[X]$. É chamada de ideal das identidades ( $T$-ideal) da classe das álgebras $\mathcal{M}$ e é denotado por $T(\mathcal{M})$.

Definição 2.2.2 (Variedade). Seja $I$ um subconjunto de $\Phi[X]$. A classe $\mathcal{M}$ de todas as $\Phi$-álgebras satisfazendo cada identidade de $I$ é chamada de variedade de $\Phi$-álgebras definida pelo conjunto de identidades $I$. I é também chamado de conjunto de definição das identidades da variedade $\mathcal{M}$.

Uma equivalência do conceito de variedade pode ser dada a seguir:

Teorema 2.2.1 (Birkhoff). Uma classe de $\Phi$-álgebras $\mathcal{V}$ é uma variedade se, e somente se, as seguintes condições são satisfeitas:

i) $\mathcal{V}$ é fechada por subálgebras, isto é, se $A \in \mathcal{V}$ e $B \subset A$ é uma subálgebra, então $B \in \mathcal{V}$.

ii) $\mathcal{V}$ é fechada por produtos diretos, isto é, se $\left\{A_{\alpha}\right\} \subset \mathcal{V}$, então $\prod_{\alpha} A_{\alpha} \in \mathcal{V}$.

iii) $\mathcal{V}$ é fechada por imagens homomórficas, isto é, se $A \in \mathcal{V}$ e $\theta: A \rightarrow B$ é um $\Phi$-homomorfismo de álgebras, então $\theta(A) \in \mathcal{V}$.

Exemplo 2.2.2. A variedade que consiste apenas na álgebra (0) é chamada de variedade trivial.

Exemplo 2.2.3. A variedade definida pela identidade $f=(x, y, z)$ é a variedade das álgebras associativas e será denotada por Assoc.

Exemplo 2.2.4. A variedade definida pela identidade $f=[x, y]$ é a variedade das álgebras comutativas e será denotada por Com.

Exemplo 2.2.5. A variedade definida pelas identidades $f_{1}=(x, x, y)$ e $f_{2}=(x, y, y)$ define a variedade das álgebras alternativas e será denotada por Alt.

Exemplo 2.2.6. A variedade definida pelas identidades $f_{1}=[x, y]$ e $f_{2}=\left(x^{2}, y, x\right)$ define a variedade das álgebras de Jordan e será denotada por Jord.

Exemplo 2.2.7. A variedade definida pelas identidades $f_{1}=(x, y, x)$ e $f_{2}=\left(x^{2}, y, x\right)$ define a variedade das álgebras de Jordan não comutativas e será denotada por NCJ.

Exemplo 2.2.8 ((-1,1)-álgebras). A variedade definida pelas identidades $f_{1}=(x, y, z)+$ $(y, z, x)+(z, x, y)$ e $f_{2}=(x, y, y)$ define a variedade das (-1,1)-álgebras e será denotada por $[-1,1]^{1}$.

\footnotetext{
${ }^{1} \mathrm{~A}$ variedade das $(-1,1)$-álgebras é uma variedade de álgebras alternativas à direita que satisfazem a identidade cíclica associativa.
} 
Exemplo 2.2.9 (Álgebras de Lie). A variedade definida pelas identidades $f_{1}=x(y z)+$ $y(z x)+z(x y)$ e $f_{2}=x y+y x$ define a variedade das álgebras de Lie e será denotada por Lie. Observe que álgebras de Lie são anticomutativas.

Todo polinômio não associativo $f \in \Phi[X]$ pode ser unicamente decomposto em uma soma de monômios irredutíveis ${ }^{2}$. Vamos dizer que um monômio $\alpha v$, em que $\alpha \in \Phi$ e $v \in V[X]$, tem tipo $\left[n_{1}, n_{2}, \cdots, n_{k}\right]$ se a palavra não associativa $v$ contém $x_{i}$ exatamente $n_{i}$ vezes e também $n_{k} \neq 0$, mas $n_{j}=0$, para $j>k$. Por exemplo, o monômio $\left(\left(x_{1} x_{3}\right) x_{3}\right)\left(x_{1} x_{4}\right)$ tem tipo $[2,0,2,1]$. Chamaremos de $n_{i}$ o grau do monômio $\alpha v$ em $x_{i}$. Se todos os monômios em uma listagem irredutível do polinômio $f$ têm o mesmo grau $n_{i}$ em $x_{i}$, então diremos que o polinômio $f$ é homogêneo em $x_{i}$ de grau $n_{i}$. Um polinômio não associativo (identidade de uma álgebra) é chamado de homogêneo se todos os seus monômios numa listagem irredutível são do mesmo tipo. Em outras palavras, um polinômio $f$ é homogêneo se há homogeneidade em cada variável. Por exemplo, o polinômio $\left(x_{1}^{2} x_{3}\right) x_{4}+\left(\left(x_{1} x_{4}\right) x_{3}\right) x_{1}$ é homogêneo, pois cada um dos seus monômios tem tipo $[2,0,1,1]$. O polinômio $x_{1}^{2} x_{2}-$ $\left(x_{1} x_{3}\right) x_{1}$ é homogêneo em $x_{1}$ de grau 2, no entanto, não é homogêneo. Um polinômio homogêneo do tipo $\left[n_{1}, n_{2}, \ldots, n_{k}\right]$, em que $n_{j} \leq 1, j=1, \cdots, k$, é dito ser multilinear.

O grau de um polinômio não associativo em $x_{i}$ é definido como o máximo grau em $x_{i}$ dos seus monômios. Seja $f \in \Phi[X]$ um polinômio arbitrário não associativo. Se reagruparmos os monômios com o mesmo tipo do polinômio $f$, então $f$ é representado na forma de uma soma de polinômios homogêneos. Estes polinômios homogêneos são chamados de componentes homogêneas do polinômio $f$.

Definição 2.2.3 (Variedade homogênea). Sejam $\mathcal{V}$ uma variedade e $f \in T(\mathcal{V})$. A variedade $\mathcal{V}$ é dita ser homogênea se todas as componentes homogêneas também estiverem em $T(\mathcal{V})$.

Os seguintes resultados podem ser encontrados em [ZSSS82].

Lema 2.2.10. Para a homogeneidade de uma variedade $\mathcal{V}$, é necessário e suficiente que $T(\mathcal{V})$, como um ideal da álgebra $\Phi[X]$, tenha um sistema de elementos geradores homogêneos.

Teorema 2.2.4. Uma variedade de álgebras sobre um corpo infinito é homogênea.

Uma variedade que interessa ao estudo, nessa parte introdutória, é a variedade de álgebras associativas, isto é, a variedade definida pela identidade $f=(x, y, z)$.

Nas seções 2.4 e 2.5, tornaremos a falar de variedades e identidades polinomiais.

\subsection{Elementos da Teoria Geral de Radicais}

Na teoria de anéis, o conceito de radical é um dos mais importantes a ser desenvolvido. Suponha que seja necessário descrever os anéis de alguma classe de anéis $\mathcal{R}$, por exemplo, a classe das $\Phi$-álgebras de Jordan. Como regra, uma classe $\mathcal{C}$ de álgebras apresenta

\footnotetext{
${ }^{2}$ Para maiores detalhes, consulte [ZSSS82]
} 
heterogeneidade entre seus elementos (do ponto de vista estrutural) e, nesta perspectiva, algumas álgebras serão tidas como "boas" e outras como "ruins". Por simplificação, suponhamos que $\mathcal{C}$ seja uma classe de anéis. Ela pode conter anéis de divisão e anéis nilpotentes, de modo a encontrar as características gerais na estrutura da classe $\mathcal{C}$, as quais, à primeira vista, revelam-se impossíveis de ser detectadas apenas pela descrição dos seus elementos. Neste caso, procedemos da seguinte forma. Em cada um dos anéis $K \in \mathcal{R}$, vamos fixar um ideal $W(K)$, chamado radical, de modo que, isoladamente, os radicais $W(K)$ e os anéis quocientes $K / W(K)$ têm uma estrutura bem definida e, em seguida, descreve-se as classes de anéis $\mathcal{R}=\{W(K)\}$ e $\mathrm{P}=\{K / W(K)\}$. Assim, um anel arbitrário da classe $\mathcal{C}$ é descrito como a extensão de um anel de $\mathcal{R}$, por intermédio de um anel de P. A utilização deste método, de forma pioneira, é devida a Wedderburn. Em 1908, ele provou que cada álgebra associativa de dimensão finita é a extensão de uma soma direta de álgebras de matrizes sobre anéis de divisão por meio de uma álgebra nilpotente.

Qualquer demonstração ou consideração histórica ocultada nesta seção poderá ser encontrada em [ZSSS82], [McCr04], [Div64] ou [GW04].

Em 1953 Kurosh e Amitsur, independentemente, lançaram as bases para um estudo axiomático do conceito de radical. Tendo como objetivo a descrição da estrutura de variedades não matriciais acerca de certas classes de álgebras não associativas, não nos aprofundaremos na Teoria de Radicais; tão somente nos restringiremos a um mínimo necessário de informação sobre a mesma.

Fixemos uma classe não vazia de $\Phi$-álgebras $\mathcal{C}$, que é fechada no que diz respeito a ideais e imagens homomórficas. No decorrer desta seção, vamos supor que todas as álgebras consideradas pertencem a $\mathcal{C}^{3}$. Diremos que $A$ é uma $\mathcal{C}$-álgebra caso ela seja um elemento de $\mathcal{C}$. Um ideal $I$ de $A$ é um $\mathcal{C}$-ideal de $A$ se $I$ estiver em $\mathcal{C}$.

Definição 2.3.1 (Álgebra semissimples). Seja $\mathcal{C}$ uma classe não vazia de álgebras. Uma álgebra $A$ é dita ser $\mathcal{C}$-semissimples se ela não contiver nenhum $\mathcal{C}$-ideal não nulo.

Se $\mathcal{C}$ é uma classe não vazia de álgebras, a classe de todos as álgebras $\mathcal{C}$-semissimples será denotada por $\mathcal{S}(\mathcal{C})$ ou $\mathcal{S}_{\mathcal{C}}$.

Definição 2.3.2 (Classe radical). Seja $\mathcal{C}$ é uma classe não vazia de álgebras. Uma classe não vazia $\mathcal{R} \subset \mathcal{C}$ de álgebras é dita ser radical se ela satisfizer as seguintes condições:

$R 1) \mathcal{R}$ é homorficamente fechada;

R2) $\mathcal{R}(A)=\sum\{J ; J \unlhd A$ e $J \in \mathcal{R}\} \in \mathcal{R}, \forall A \in \mathcal{C}$;

R3) $\mathcal{R}(A / \mathcal{R}(A))=(0)$, para qualquer álgebra $A \in \mathcal{C}$.

A condição $R 1$ ) diz que se $\varphi$ é um $\Phi$-homomorfismo (de álgebras) qualquer definido sobre $A \in \mathcal{R}$, então $\varphi(A) \in \mathcal{R}$. Tendo em mente a correspondência entre ideais e imagens homomórficas de uma álgebra; isso significa que podemos proceder de acordo com esta identificação da seguinte forma: se $I \unlhd A$ e $A \in \mathcal{R}$, então $A / I \in \mathcal{R}$. Em particular, se $A \in \mathcal{R}$ e $A$ é isomorfo à álgebra $B$, então $B \in \mathcal{R}$. O ideal $\mathcal{R}(A)$, que existe para qualquer álgebra $A \in \mathcal{C}$, é chamado de $\mathcal{R}$-radical da álgebra $A$. Uma classe radical será chamada simplesmente de radical e, em virtude da observação anterior, note que "radical" e "radical de uma álgebra" são conceitos bastante distintos, pois "radical" é uma classe de álgebras

\footnotetext{
${ }^{3}$ É importante nos lembrarmos de que a coleção de todos as álgebras não é um conjunto (consulte [Jec03]).
} 
satisfazendo certas propriedades, ao passo que o "radical de uma álgebra" é um ideal da álgebra que pertence a uma certa classe radical (quando esse mesmo ideal passa a ser visto, isoladamente, como uma álgebra).

O próximo teorema é uma maneira menos intuitiva de se definir uma classe radical. Entretanto, é uma forma equivalente da definição 2.3.2 e bastante útil na prova de alguns resultados sobre radicais.

Teorema 2.3.3. A classe de álgebras $\mathcal{R}$ é radical se, e somente se, ela satisfizer:

R1) $\mathcal{R}$ é homorficamente fechada;

R4) Se toda imagem homomórfica não nula de uma álgebra $A$ contém um $\mathcal{R}$-ideal não nulo, então A está em $\mathcal{R}$.

Demonstração. Suponha que $\mathcal{R}$ seja um radical. Vejamos que $R 2$ ) e $R 3$ ) implicam $R 4$ ): façamos por contraposição. Seja $A$ uma álgebra não nula que não está em $\mathcal{R}$. Por $R 2$ ), existe um $\mathcal{R}$-ideal maximal $\mathcal{R}(A) \neq A$ contido em $A$. Ademais, $A / \mathcal{R}(A)$ é uma imagem epimorfa não nula de $A$. Por $R 3), A / \mathcal{R}(A)$ não deve conter um $\mathcal{R}$-ideal não nulo. Concluímos, portanto, que existe uma imagem homomórfica não nula de $A$ que não contém nenhum $\mathcal{R}$-ideal.

Assuma agora que $\mathcal{R}$ cumpre as condições $R 1$ ) e $R 4$ ). Observemos que (0) é um $\mathcal{R}$ ideal, por argumento de vacuidade com respeito à condição $R 4$ ). Para estabelecer $R 2$ ), seja $J$ a soma de todos os $\mathcal{R}$-ideais de uma álgebra $A$. Mostraremos que $J$ é um $\mathcal{R}$-ideal de $A$. Se $J=(0)$, segue-se o fato trivialmente. Se $J \neq(0)$, seja $J / K$ uma álgebra quociente não nula qualquer. Como $K \subsetneq J$, deve existir em $A$ um $\mathcal{R}$-ideal $W$ tal que $W$ não está contido em $K$ (pois se assim não fosse, $K$ seria o maximal dos $\mathcal{R}$-ideais). Pelo segundo teorema do isomorfismo

$$
(W+K) / K \cong W /(W \cap K) .
$$

O lado esquerdo desse isomorfismo é um ideal não nulo de $J / K$, enquanto o lado direito é uma imagem homomórfica da $\mathcal{R}$-álgebra $W$ e, por $R 1$ ), é também uma $\mathcal{R}$-álgebra. Portanto, toda imagem homomórfica não nula de $J$ contém um $\mathcal{R}$-ideal não nulo. Por $R 4), J$ é uma $\mathcal{R}$-álgebra. Isso estabelece $R 2$ ).

Finalmente, devemos estabelecer $R 3)$. Seja $A$ uma $\Phi$-álgebra qualquer. Sabemos que $A$ tem um $\mathcal{R}$-radical $\mathcal{R}(A)$, pois $R 2$ ) já é estabelecido. Suponha que $A / \mathcal{R}(A)$ não seja uma álgebra $\mathcal{R}$-semissimples e seja $\mathcal{R}(A / \mathcal{R}(A))=M / \mathcal{R}(A) \neq(0)$. Ora, $M$ é um ideal de $A$ e $M$ contém propriamente $\mathcal{R}(A)$. Seja $M / N$ uma álgebra quociente (arbitrária) de $M$. Se $N \supseteq \mathcal{R}(A)$, então $\mathcal{R}(A)$ é um ideal de $N$ e, pelo terceiro teorema do isomorfismo,

$$
M / N \cong(M / \mathcal{R}(A)) /(N / \mathcal{R}(A))
$$

e, por $R 1), M / N$ é uma $\mathcal{R}$-álgebra. Entretanto, se $N \nsupseteq \mathcal{R}(A)$, então $\mathcal{R}(A) \cap N \subset \mathcal{R}(A)$ e, pelo segundo teorema do isomorfismo,

$$
(N+\mathcal{R}(A)) / N \cong \mathcal{R}(A) /(\mathcal{R}(A) \cap N)
$$

O lado esquerdo desse isomorfismo é um ideal não nulo de $M / N$, enquanto o lado direito é uma imagem homomórfica de $\mathcal{R}(A)$ e, por $R 1$ ), é também está em $\mathcal{R}$. Portanto, toda imagem homomórfica não nula de $M$ contém um $\mathcal{R}$-ideal não nulo e, por $R 4$ ), $M$ é uma $\mathcal{R}$-álgebra, pela qual concluímos que $M$ deve estar contido em $\mathcal{R}(A)$ : contradição! Isso estabelece $R 3$ ) e encerra a prova. 
Exibiremos outra forma equivalente de se definirem classes radicais, usando aquilo que chamamos de fecho por extensão.

Teorema 2.3.4. A classe de álgebras $\mathcal{R}$ é radical se, e somente se, ela satisfizer as condições abaixo:

R1) $\mathcal{R}$ é homomorficamente fechada;

R2) $\mathcal{R}(A)=\sum\{J ; J \unlhd A$ e $J \in \mathcal{R}\} \in \mathcal{R}$, para qualquer álgebra $A \in \mathcal{C}$;

R5) (Fecho por extensões) $S e I \unlhd A$ e $I, A / I \in \mathcal{R}$, então $A \in \mathcal{R}$.

Demonstração. Assuma que $\mathcal{R}$ é radical. Sejam $A$ uma álgebra arbitrária e $I$ um ideal de $A$ tais que $I$ e $A / I$ estejam em $\mathcal{R}$, isto é, $\mathcal{R}(I)=I$ e $\mathcal{R}(A / I)=A / I$. É claro que $\mathcal{R}(I) \subseteq \mathcal{R}(A)$, pois $\mathcal{R}$-ideais de $A$ contidos em $I$ também são $\mathcal{R}$-ideais de $A$, para qualquer álgebra $A$ e, nesse caso, como $I$ é um $\mathcal{R}$-ideal de $A$, isso significa que $I$ é ideal de $\mathcal{R}(A)$. Ademais,

$$
\frac{A / I}{\mathcal{R}(A) / I} \cong A / \mathcal{R}(A)
$$

$\log 0$

$$
\frac{\mathcal{R}(A / I)}{\mathcal{R}(A) / I}=\mathcal{R}\left(\frac{A / I}{\mathcal{R}(A) / I}\right)=\mathcal{R}(A / \mathcal{R}(A))=(0)
$$

e significa que $\mathcal{R}(A / I)=\mathcal{R}(A) / I$. Portanto,

$$
A / I=\mathcal{R}(A / I)=\mathcal{R}(A) / I,
$$

implicando que $A=\mathcal{R}(A)$ ou, equivalentemente, $A \in \mathcal{R}$, pela qual concluímos que $\mathcal{R}$ satisfaz a condição $R 5)$. Reciprocamente, suponha que $\mathcal{R}$ satisfaça as condições $R 1$ ), $R 2$ ) e $R 5)$. Se $A \in \mathcal{R}$, então é óbvio que $A / \mathcal{R}(A)=A / A=(0)$. Seja então uma álgebra $A \notin \mathcal{R}$. Pela contraposição da condição $R 5), A / \mathcal{R}(A) \notin \mathcal{R}$. Suponha agora que $\mathcal{R}(A / \mathcal{R}(A)) \neq(0)$, digamos $\mathcal{R}(A / \mathcal{R}(A))=M / \mathcal{R}(A)$. Logo, $M$ é um $\mathcal{R}$-ideal próprio de $A$, pois $\mathcal{R}$ satisfaz $R 5)$, contendo propriamente $\mathcal{R}(A)$, contradizendo a maximalidade de $\mathcal{R}(A)$ e, portanto, a condição $R 2)$. Logo, $\mathcal{R}(A / \mathcal{R}(A))=(0)$, para uma álgebra $A$ arbitrária, implicando no fato de que $\mathcal{R}$ cumpre as condições $R 1), R 2$ ) e $R 3)$; portanto $\mathcal{R}$ é radical.

Outra caracterização para classes radicais é a seguinte:

Teorema 2.3.5. A classe de álgebras $\mathcal{R}$ é radical se, e somente se, ela satisfizer as condições abaixo:

R1) $\mathcal{R}$ é homomorficamente fechada;

R6) (Propriedade indutiva) $\mathcal{R}$ contém todas as uniões de cadeias de $\mathcal{R}$-ideais de uma álgebra A arbitrária;

R5) (Fecho por extensões) $S e I \unlhd A$ e $I, A / I \in \mathcal{R}$, então $A \in \mathcal{R}$. 
Consulte [GW04].

O próximo lema nos permite afirmar quando uma álgebra é $\mathcal{R}$-radical ou não usando a $\mathcal{R}$-semissimplicidade.

Lema 2.3.1. Seja $\mathcal{R}$ uma classe radical. A é uma álgebra $\mathcal{R}$-radical se, e somente se, $A$ não pode ser sobrejetado homomorficamente numa álgebra $\mathcal{R}$-semissimples não nula.

Demonstração. Se $A$ é uma álgebra $\mathcal{R}$-radical, então, por $R 1$ ), toda imagem homomórfica não nula de $A$ é também uma álgebra $\mathcal{R}$-radical e, portanto, não é $\mathcal{R}$-semissimples. Para provar a recíproca, suponha que $A$ não seja uma álgebra $\mathcal{R}$-radical. Por $R 2$ ), existe o $\mathcal{R}$-radical $\mathcal{R}(A) \neq A$. Logo, $A$ pode ser sobrejetado homorficamente na álgebra $A / \mathcal{R}(A)$, a qual, por $R 3$ ), é $\mathcal{R}$-semissimples.

Esse lema afirma que álgebras $\mathcal{R}$-radicais e $\mathcal{R}$-semissimples são bastante distintas com respeito às suas propriedades algébricas. Mais adiante, entenderemos com mais clareza essa "distinção radical" entre as álgebras da classe radical $\mathcal{R}$ e as álgebras $\mathcal{R}$-semissimples em $S(\mathcal{R})$.

Definição 2.3.6 (Hereditariedade). Uma classe de álgebras $\mathcal{C}$ é dita hereditária à direita (à esquerda) se para qualquer $A \in \mathcal{C}$ e para qualquer $I$ ideal à direita (à esquerda) de $A$ tem-se $I \in \mathcal{C}$. Uma classe é hereditária se ela for hereditária com respeito aos ideais bilaterais.

Teorema 2.3.7. Um radical $\mathcal{R}$ é hereditário se, e somente se, para qualquer ideal I de uma $\Phi$-álgebra $A$ arbitrária tem-se que se $B \in \mathcal{R}$ e $I$ é um ideal de $B$, então $I \in \mathcal{R}$, em que $\mathcal{R}$ é a classe semissimples com respeito ao radical $\mathcal{R}$.

Vamos considerar exemplos concretos de radicais que nos serão úteis neste trabalho.

\subsubsection{Exemplos de radicais}

No Exemplo 2.1.10, definimos as álgebras de potências associativas. São exemplos de álgebras de potências associativas:

- as álgebras associativas (Exemplo 2.1.9), trivialmente;

- as álgebras de Jordan (Exemplo 2.1.11);

- as álgebras alternaivas (Exemplo 2.1.12);

- as (-1,1)-álgebras (Exemplo 2.2.8);

- as álgebras de Jordan não comutativas (Exemplo 2.1.14).

Seja A uma álgebra de potências associativas. Consideremos a seguinte definição:

Definição 2.3.1.1 (Álgebras nil e nilpotentes). Dada uma álgebra A, um elemento $b$ é dito ser nilpotente ou um nil elemento se $b^{k}=0$, para algum natural $k$. Seja $m$ o menor natural tal que $b^{m}=0$. Tal $m$ será chamado de indice de nilpotência do elemento $b$. Uma álgebra é nil se para todo $c \in \mathbf{A}$, existir um inteiro $m=m(c)$ tal que $c^{m}=0$. Uma 
álgebra A é chamada de nilpotente se existe um número natural $r$ tal que o produto de quaisquer $r$ elementos da álgebra $\mathbf{A}$, com qualquer arranjo (permitido) de parênteses, é igual a zero, isto é, se $\mathbf{A}^{r}=(0)$. Seja $n$ o menor natural tal que $\mathbf{A}^{n}=(0)$. Tal $n$ será chamado de indice de nilpotência da álgebra $\mathbf{A}$.

Uma álgebra de potências associativas nil para a qual os índices de nilpotência de todos os elementos são limitados por algum inteiro $n$ satisfaz a identidade $f(x)=x^{n}$. Chamaremos tal álgebra de álgebra nil de indice limitado.

Se $x$ é um elemento de uma álgebra de potências associativas e $m$ é um número natural,

$$
x^{m}=\underbrace{x \cdot x \cdot \ldots \cdot x}_{m \text {-vezes }}
$$

está bem definido.

Definição 2.3.1.2 (Nilpotência local). Dizemos que A é uma álgebra localmente nilpotente se qualquer conjunto finito de elementos gera uma subálgebra nilpotente. Em outras palavras, a nilpotência local pode ser expressa através da seguinte propriedade:

$$
\left(a_{1}, \cdots, a_{n} \text { são elementos de } \mathbf{A}\right) \Longrightarrow\left(\operatorname{alg}\left\langle a_{1}, \cdots, a_{n}\right\rangle \text { é nilpotente }\right) \text {. }
$$

Definição 2.3.1.3 (Álgebras algébricas). Sejam $F$ um corpo e A uma $F$-álgebra de potências associativas. Um elemento $a \in \mathbf{A}$ é dito ser algébrico se existe um número natural $n$ tal que

$$
a^{n}=\sum_{i<n} \alpha_{i} a^{i}, \quad \alpha_{i} \in F .
$$

O menor número $n$ atendendo a condição anterior é chamado de grau algébrico do elemento a.

A é dita ser algébrica se todos os seus elementos são algébricos.

Definição 2.3.1.4 (Dimensão finita local). Sejam $F$ um corpo e e A uma $F$-álgebra de potências associativas. Dizemos que A é uma álgebra localmente de dimensão finita se qualquer conjunto finito de elementos gera uma subálgebra de dimensão finita (dimensão de $F$-espaço vetorial). Em outras palavras, a dimensão finita local pode ser expressa através da seguinte propriedade:

$$
\left(a_{1}, \cdots, a_{n} \text { são elementos de } \mathbf{A}\right) \Longrightarrow\left(\operatorname{dim}_{F}\left(\operatorname{alg}\left\langle a_{1}, \cdots, a_{n}\right\rangle\right)<\infty\right) .
$$

Vamos a um conceito que também nos será útil na determinação da estrutura de certas álgebras.

Definição 2.3.1.5 (Álgebras primas). Uma álgebra A é dita ser prima se, para quaisquer ideais $I, J \unlhd \mathbf{A}$, a seguinte propriedade se verifica:

$$
I J=(0) \Longrightarrow I=(0) \text { ou } J=(0) .
$$

Lembremos que $I J$ é o produto de ideais da Definição 2.1.3.

Por sua vez, um ideal $I \unlhd \mathbf{A}$ é primo se $\mathbf{A} / I$ é uma álgebra prima.

Definição 2.3.1.6 (Álgebras simples). Uma álgebra $\mathbf{A}$ é dita ser simples se $\mathbf{A}^{2} \neq(0)$ e os únicos ideais de $\mathbf{A}$ são (0) e $\mathbf{A}$. 
Introduziremos alguns radicais que serão úteis nesta tese, apresentados originalmente em [ZSh73].

\section{Os radicais alg (algébrico) e Nil (nil radical)}

Sejam $\Phi$ um anel de escalares e $Z \unlhd \Phi$ um ideal de $\Phi$.

Definição 2.3.1.7 (Álgebras $Z$-algébricas). Sejam $\Phi$ um anel de escalares, $Z \unlhd \Phi$ um ideal e $\mathbf{A}$ uma $\Phi$-álgebra de potências associativas. Um elemento $a \in \mathbf{A}$ é dito ser algébrico sobre $Z$ ou $Z$-algébrico se existe um número natural $n$ tal que

$$
a^{n}=\sum_{i<n} z_{i} a^{i}, \quad z_{i} \in Z
$$

O menor número $n$ atendendo a condição anterior é chamado de grau algébrico do elemento $a$.

A é dita ser $Z$-algébrica se todos os seus elementos são $Z$-algébricos.

Seja A uma álgebra de potências associativas. Um ideal $I \unlhd \mathbf{A}$ é dito ser algébrico sobre $Z$ (ou $Z$-algébrico) se, quando visto isoladamente, $I$ é uma álgebra $Z$-algébrica (Definição 2.3.1.7) sobre $Z$.

Nos Lemas 2.3.1.9, 2.3.1.10, 2.3.1.11 e 2.3.1.8, a seguir, A é uma álgebra de potências associativas.

Lema 2.3.1.8. Seja I um ideal de $\mathbf{A}$ tal que I e $\mathbf{A} / I$ são álgebras Z-algébricas. Então A é também álgebra Z-algébrica.

Demonstração. Seja $a \in \mathbf{A}$ um elemento arbitrário. Por hipótese, $\bar{a}=a+I$ é $Z$-algébrico, isto é, existe $m$ natural tal que

$$
\overline{a^{m}}=\bar{a}^{m}=\sum_{i<m} z_{i} \bar{a}^{i}=\overline{\sum_{i<m} z_{i} a^{i}}, \quad z_{i} \in Z .
$$

Como as classes de $a^{m}$ e $\sum_{i<m} z_{i} a^{i}$ coincidem, o elemento $b=a^{m}-\sum_{i<m} z_{i} a^{i}$ está em $I$. Por hipótese, $b$ é $Z$-algébrico também, logo existe $n$ tal que

$$
b^{n}=\sum_{j<n} z_{j}^{\prime} b^{j}, \quad z_{j}^{\prime} \in Z
$$

Ora,

$$
\left(a^{m}-\sum_{i<m} z_{i} a^{i}\right)^{n}=\sum_{j<n} z_{j}^{\prime}\left(a^{m}-\sum_{i<m} z_{i} a^{i}\right)^{j}
$$

portanto

$$
a^{m n}=\sum_{k<m n} z_{k} a^{k}
$$

Em outras palavras, $a$ é $Z$-algébrico e, como $a \in \mathbf{A}$ é arbitrário, concluímos que $\mathbf{A}$ é $Z$-algébrica.

Lema 2.3.1.9. Seja $f: \mathbf{A} \rightarrow B$ um homomorfismo de $\Phi$-álgebras. Se $\mathbf{A}$ é Z-algébrica, então imf $\subseteq B$ também é $Z$-algébrica. 
Lema 2.3.1.10. Sejam $I_{1}, I_{2} \unlhd \mathbf{A}$ ideais $Z$-algébricos. Então, $I_{1}+I_{2}$ é um ideal $Z$ algébrico.

De forma indutiva, o seguinte resultado por ser estabelecido:

Lema 2.3.1.11. Seja $J=\sum\{I \unlhd \mathbf{A} \mid I$ é Z-algébrico $\}$. Então $J \unlhd \mathbf{A}$ é um ideal Z-algébrico.

De acordo com o Teorema 2.3.4, a classe das álgebras $Z$-algébricas é uma classe radical (na classe das $\Phi$-álgebras de potências associativas). Defina

$$
\mathfrak{a l g}_{Z}(\mathbf{A})=\sum\{I \unlhd \mathbf{A} \mid I \text { é } Z \text {-algébrico }\},
$$

ou seja, $\mathfrak{a l g}_{Z}(\mathbf{A})$ é o ideal maximal $Z$-algébrico de $\mathbf{A}$ estabelecido no Lema 2.3.1.11.

$\mathrm{O}$ ideal $\mathfrak{a l g}_{Z}(\mathbf{A})$ será chamado de radical $Z$-algébrico de $\mathbf{A}$ e, como A é uma álgebra de potências associativas arbitrária, concluímos o seguinte:

Teorema 2.3.1.12. Se A é uma álgebra de potências associativas, então o seu radical $Z$-algébrico $\mathfrak{a l g}_{z}(\mathbf{A})$ está definido. Em particular, $\mathfrak{a l g}_{Z}\left(\mathbf{A} / \mathfrak{a l g}_{Z}(\mathbf{A})\right)=(\overline{0})$.

Ademais, a estrutura das álgebras $\mathfrak{a l g}_{Z}$-semissimples é dada como a seguir:

Teorema 2.3.1.13 (Shestakov [ZSh73]). Se A é uma álgebra de potências associativas, então $\mathbf{A} / \mathfrak{a l g}_{z}(\mathbf{A})$ é isomorfa a uma soma subdireta de álgebras primas $\mathfrak{a l g}_{Z}$-semissimples:

$$
\mathbf{A} / \mathfrak{a} \mathfrak{g}_{Z}(\mathbf{A}) \hookrightarrow \prod \mathbf{A}_{\alpha}, \quad \mathbf{A}_{\alpha} \text { é prima e } \mathfrak{a l g}_{Z}\left(\mathbf{A}_{\alpha}\right)=(0), \forall \alpha .
$$

Corolário 2.3.1.14. $S e \Phi=F$ é um corpo, A é uma $F$-álgebra de potências associativas e $Z=F$ no Teorema 2.3.1.12, então a classe das álgebras algébricas (Definição 2.3.1.3) é radical (na classe das álgebras de potências associativas). Denotemos o radical F-algébrico por $\mathfrak{a l g}_{F}(\mathbf{A})$.

Se o corpo $F$ em questão já estiver explícito num determinado contexto, escreveremos apenas radical algébrico e o notaremos por $\mathfrak{a l} \mathfrak{g}(\mathbf{A})$.

Corolário 2.3.1.15. Se A é uma $\Phi$-álgebra de potências associativas e $Z=(0)$ no Teorema 2.3.1.12, então a classe das álgebras zero algébricas é radical (na classe das álgebras de potências associativas). Denotemos o radical zero algébrico de $\mathbf{A}$ por $\mathfrak{a l g}_{0}(\mathbf{A})$.

Um elemento zero algébrico é, tão somente, um elemento nilpotente e uma álgebra zero algébrica é, tão somente, uma álgebra nil (Definição 2.3.1.1). 
Portanto, a classe das álgebras nil é radical (na classe das álgebras de potências associativas). Dessa forma, estabelecemos uma nova notação ao radical $\mathfrak{a} \mathfrak{l g}_{0}(\mathbf{A})$ :

$$
\operatorname{Nil}(\mathbf{A}):=\mathfrak{a l g}_{0}(\mathbf{A})
$$

Reescreveremos o Corolário 2.3.1.15 da seguinte forma:

Corolário 2.3.1.16. Se A é uma $\Phi$-álgebra de potências associativas, então a classe das álgebras nil é radical (na classe das álgebras de potências associativas). Denotemos o nil radical de $\mathbf{A}$ por $\mathbf{N i l}(\mathbf{A})$.

Podemos reescrever o Teorema 2.3.1.13 das seguintes formas:

Corolário 2.3.1.17 (Shestakov [ZSh73]). Se A é uma álgebra de potências associativas, então $\mathbf{A} / \mathfrak{a} \mathfrak{l g}_{F}(\mathbf{A})$ é isomorfa a uma soma subdireta de álgebras primas $\mathfrak{a l g}_{F^{-}}$-semissimples:

$$
\mathbf{A} / \mathfrak{a l} \mathfrak{g}_{F}(\mathbf{A}) \hookrightarrow \prod \mathbf{A}_{\alpha}, \quad \mathbf{A}_{\alpha} \text { é prima e } \mathfrak{a l} \mathfrak{g}_{F}\left(\mathbf{A}_{\alpha}\right)=(0), \forall \alpha .
$$

Corolário 2.3.1.18 (Shestakov [ZSh73]). Se A é uma álgebra de potências associativas, então A/Nil(A) é isomorfa a uma soma subdireta de álgebras primas Nil-semissimples:

$$
\mathbf{A} / \operatorname{Nil}(\mathbf{A}) \hookrightarrow \prod \mathbf{A}_{\alpha}, \quad \mathbf{A}_{\alpha} \text { é prima e } \operatorname{Nil}\left(\mathbf{A}_{\alpha}\right)=(0), \forall \alpha .
$$

Agora, investigaremos o análogo desta construção de maneira local.

\section{Os radicais $\mathcal{L}_{Z}$ (Z-localmente finito) e Locnil (localmente nilpotente)}

Continuamos mantendo a notação adotada na construção anterior. $\Phi$ é o anel de escalares, A é uma $\Phi$-álgebra de potências associativas e $Z$ é um ideal de $\Phi$.

Diremos que uma $\Phi$-álgebra A é finita sobre $Z$ se existem elementos $a_{1}, a_{2}, \cdots a_{k}$ em A tais que para algum número natural $m$, cada elemento $c \in \mathbf{A}^{m}$ admite uma representação na forma $c=\sum_{i=1}^{k} z_{i} a_{i}$, em que $z_{i} \in Z$.

Suponha que, para uma $\Phi$-álgebra $\mathbf{A}$, seja verdade que qualquer conjunto finito de $\mathbf{A}$ gera uma $\Phi$-álgebra finita sobre $Z$. Nessas condições, diremos que a álgebra $\mathbf{A}$ é localmente finita sobre $Z$.

Um ideal $I \unlhd \mathbf{A}$ é dito ser localmente finito sobre $Z$ se, isoladamente, $I$ é uma álgebra localmente finita sobre $Z$.

Em particular, quando $Z=(0)$ a álgebra finita sobre $Z$ é nilpotente e, neste caso, a finitude local sobre $Z$ transforma-se em nilpotência local. Quando $Z=\Phi$ e $\Phi=F$ é um corpo, então a álgebra finita sobre $Z$ é a álgebra de dimensão finita sobre $F$ e a finitude local sobre $Z$ torna-se a dimensão finita local usual (Definição 2.3.1.4).

Vamos repetir o procedimento padrão adotado anteriormente, com uma diferença: nem sempre a classe de álgebras localmente finitas é radical. Por exemplo, a classe de álgebras localmente finitas não é radical na variedade das álgebras de Lie.

Em [ZSh73], Shestakov e Zhevlakov estabelecem critérios (com razoável grau de generalização) para os quais uma variedade de álgebras possa admitir um radical localmente finito.

Seja $\mathcal{V}^{L}$ uma variedade de álgebras que satisfaz as propriedades abaixo: 
I) Se $\mathbf{A} \in \mathcal{V}^{L}$ e $I$ é um ideal de $A$, então $I^{3} \subseteq I^{2}$.

II) Se $\mathbf{A} \in \mathcal{V}^{L}$, então qualquer elemento da álgebra de multiplicações $M$ da álgebra A da forma $\sigma=T_{a} T_{b} T_{a}^{\prime}$ pode ser representado como uma combinação linear de elementos de $M$ na forma $T_{a} T_{a}^{\prime} T_{b}$ e de elementos de mesma composição com respeito ao conjunto $\{a, b\}$ conforme a definição de $\sigma$, sendo da forma $T_{c} T_{d}$ e $T_{e}$. Aqui tem-se $\left.T_{x}, T_{x}^{\prime}, T_{x}^{(i)} \in\left\{R_{x}, L_{x}\right\}\right)^{4}$.

III) Se $\mathbf{A} \in \mathcal{V}^{L}$, então qualquer elemento $x$ de $\mathbf{A}$ tal que $x^{2}=0$ será fracamente Engel, isto é, para cada $y \in A$, existe $n=n(x, y)$ tal que $[x, y, \cdots, y]=0$, em que $y$ ocorre $n$ vezes nesta expressão.

Fixemos $\mathcal{V}^{L}$ com as propriedades I, II e III mencionadas acima e seja $\mathbf{A} \in \mathcal{V}^{L}$. Os autores mostram, em [ZSh73] que a variedade $\mathcal{V}^{L}$ admite o radical localmente nilpotente:

Teorema 2.3.1.19 ([ZSh73]). Se $\mathbf{A} \in \mathcal{V}^{L}$, então o seu radical localmente finito sobre $Z$ $\mathcal{L}_{Z}(\mathbf{A})$ está definido.

Teorema 2.3.1.20 (Shestakov [ZSh73]). Suponha que numa variedade $\mathcal{V}$ de $\Phi$-álgebras exista um radical $\mathcal{L}_{Z}$ que é localmente finito sobre $Z$. Nessa condição, para qualquer álgebra $\mathbf{A} \in \mathcal{V}$, o radical $\mathcal{L}_{Z}(\mathbf{A})$ será igual a intersecção $\cap P$ de todos os ideais primos da álgebra $\mathbf{A}$, de forma que a álgebra quociente $\overline{\mathbf{A}}=\mathbf{A} /(\cap P)$ não contém ideais (bilaterais) não nulos que são localmente finitos sobre $Z$ (em outras palavras, a álgebra $\overline{\mathbf{A}}$ é $\mathcal{L}_{Z^{-}}$ semissimples).

Em particular, qualquer álgebra $\mathcal{L}_{Z}$-semissimples em $\mathcal{V}$ é isomorfa a uma soma subdireta de $\Phi$-álgebras primas $\mathcal{L}_{Z}$ semissimples:

$$
\mathbf{A} / \mathcal{L}_{Z}(\mathbf{A}) \hookrightarrow \prod \mathbf{A}_{\alpha}, \quad \mathbf{A}_{\alpha} \text { é prima e } \mathcal{L}_{Z}\left(\mathbf{A}_{\alpha}\right)=(0), \forall \alpha .
$$

Observamos, no início desta construção, que se $Z=F$ é um corpo e A é uma $F$ álgebra, então os conceitos "finitude local sobre $F$ " e "dimensão finita local" coincidem.

Corolário 2.3.1.21. Se $\Phi=F$ é um corpo, $\mathbf{A} \in \mathcal{V}^{L}$ e $Z=F$ no Teorema 2.3.1.19, então a classe das álgebras de dimensão finita localmente (Definição 2.3.1.4) é radical em $\mathcal{V}^{L}$. Denotemos o radical de dimensão finita local por $\mathcal{L}_{F}(\mathbf{A})$.

Se o corpo $F$ em questão já estiver explícito em um determinado contexto notaremos o radical de dimensão finita local por $\mathcal{L}(\mathbf{A})$.

Em virtude da observação já feita no início desta construção, se $Z=(0)$, os conceitos "finitude local sobre (0)" e "nilpotência local" coincidem.

Corolário 2.3.1.22. Se A é uma $\Phi$-álgebra em $\mathcal{V}^{L}$ e $Z=(0)$ no Teorema 2.3.1.19, então a classe das álgebras localmente nilpotentes (Definição 2.3.1.2) é radical em $\mathcal{V}^{L}$. Denotemos o radical localmente nilpotente de $\mathbf{A}$ por Locnil(A).

\footnotetext{
${ }^{4}$ Para maiores deatlhes, consulte [ZSh73]
} 
Podemos reescrever o Teorema 2.3.1.20 das seguintes formas:

Teorema 2.3.1.23 (Shestakov [ZSh73]). Se $\mathbf{A} \in \mathcal{V}^{L}$, então $\mathbf{A} / \mathcal{L}_{F}(\mathbf{A})$ é isomorfa a uma soma subdireta de álgebras primas $\mathcal{L}_{F}$-semissimples:

$$
\mathbf{A} / \mathcal{L}_{F}(\mathbf{A}) \hookrightarrow \prod \mathbf{A}_{\alpha}, \quad \mathbf{A}_{\alpha} \text { é prima e } \mathcal{L}_{F}\left(\mathbf{A}_{\alpha}\right)=(0), \forall \alpha .
$$

Teorema 2.3.1.24 (Shestakov [ZSh73]). Se $\mathbf{A} \in \mathcal{V}^{L}$, então A/Locnil(A) é isomorfa a uma soma subdireta de álgebras primas Locnil-semissimples:

$$
\mathbf{A} / \operatorname{Locnil}(\mathbf{A}) \hookrightarrow \prod \mathbf{A}_{\alpha}, \quad \mathbf{A}_{\alpha} \text { é prima e Locnil }\left(\mathbf{A}_{\alpha}\right)=(0), \forall \alpha \text {. }
$$

A variedade $\mathcal{V}^{L}$ aqui descrita é deveras abstrata. Isso não se constitui um problema ao nosso trabalho, pois ainda em [ZSh73] os autores estabelecem que álgebras alternativas, álgebras de Jordan e $(-1,1)$-álgebras estão em $\mathcal{V}^{L}$. Portanto, nessas classes de álgbras, o radical localmente finito pode ser estabelecido. Mostraremos, mais adiante, que é possível estabelecer o radical localmente finito para certas classes de álgebras de Jordan não comutativas .

Os resultados exibidos nesta subseção, particularmente se A é uma álgebra em Jord $\cup$ Alt $\cup[-1,1]$, nos permitem determinar a existência dos seguintes radicais:

- $\mathrm{Nil}(\mathbf{A})$, o nil radical de A, também conhecido como nil radical superior ou radical de Köethe.

- Locnil(A), o radical localmente nilpotente de $\mathbf{A}$, também conhecido como radical de Levitzki.

- $\mathfrak{a l g}(\mathbf{A})$, o radical algébrico de $\mathbf{A}$.

- $\mathcal{L}_{Z}(\mathrm{~A})$, o radical localmente finito (sobre um certo ideal $Z$ do anel de operadores de A), que induz a existência de um radical de dimensão finita local.

Algumas inclusões precisam ser aqui mencionadas. O radical Locnil(A) é, em particular, um nil ideal de $\mathbf{A}$, logo

$$
\operatorname{Locnil}(\mathbf{A}) \subseteq \operatorname{Nil}(\mathbf{A})
$$

Se $F$ é um corpo e A é uma $F$-álgebra, então

$$
\operatorname{Nil}(\mathbf{A}) \subseteq \mathfrak{a l g}(\mathbf{A})
$$

Em virtude das observações acima, se A é uma $F$-álgebra, $F$ é um corpo, então

$$
\operatorname{Locnil}(\mathbf{A}) \subseteq \operatorname{Nil}(\mathbf{A}) \subseteq \mathfrak{a l g}(\mathbf{A})
$$

Ademais,

$$
\operatorname{Locnil}(\mathbf{A}) \subseteq \mathcal{L}(\mathbf{A})
$$


As álgebras de Jordan não comutativas são álgebras de potências associativas. Portanto, se $F$ é um corpo e A é uma $F$-álgebra de Jordan não comutativa, então

$$
\operatorname{Nil}(\mathbf{A}) \subseteq \mathfrak{a l g}(\mathbf{A})
$$

Encerramos esta seção com estes radicais, pois serão úteis a este trabalho. Há radicais mais específicos em certas classes de álgebras, todavia sua apresentação se dará nos capítulos reservados a tais classes.

\section{$2.4 \quad$ PI-álgebras associativas}

A teoria das álgebras associativas com identidades polinomiais (PI-álgebras associativas) está bem desenvolvida no presente momento e contém uma extensa quantidade de tópicos. Nesta seção, a palavra "álgebra" denotará álgebra associativa, a menos que se explicite o contrário.

Seja $\Phi$ um anel associativo e comutativo arbitrário, com elemento identidade 1 (um anel de operadores). Consideremos a álgebra livre associativa na variedade Assoc, Assoc $[X]$, sobre um conjunto enumerável de geradores $X=\left\{x_{1}, x_{2}, \cdots\right\}$. Elementos da álgebra Assoc $[X]$ são polinômios associativos das variáveis não comutativas $x_{1}, x_{2}, \ldots$, com coeficientes em $\Phi$. Chamamos um elemento $f\left(x_{1}, x_{2}, \cdots x_{n}\right) \in$ Assoc $[X]$ de admissivel sempre que um dos coeficientes para os termos de grau mais alto do polinômio $f\left(x_{1}, x_{2}, \ldots, x_{n}\right)$ é igual ao elemento identidade. Agora sejam $A$ uma $\Phi$-álgebra associativa e $M$ um $\Phi$ submódulo da álgebra $A$. Diremos que $M$ satisfaz uma identidade polinomial admissível se existe um elemento admissível $f\left(x_{1}, x_{2}, \cdots, x_{n}\right) \in \operatorname{Assoc}[X]$ de tal forma que

$$
f\left(m_{1}, m_{2}, \cdots, m_{n}\right)=0
$$

para quaisquer $m_{1}, m_{2}, \cdots, m_{n} \in M$.

Se a álgebra $A$ satisfaz uma identidade polinomial admissível $f$, então diremos que a álgebra $A$ é uma $P I$-álgebra, por brevidade.

Vejamos alguns exemplos de PI-álgebras associativas.

Exemplo 2.4.1. Qualquer álgebra comutativa é uma PI-álgebra em Assoc, pois satisfaz a identidade $f\left(x_{1}, x_{2}\right)=x_{1} x_{2}-x_{2} x_{1}$.

Considere o polinômio

$$
\left[x_{1}, \cdots, x_{n}\right]=\sum_{\sigma \in \text { Sym }_{n}}(-1)^{\operatorname{sgn} \sigma} x_{\sigma(1)} \cdots x_{\sigma(n)},
$$

em que $\sigma$ é percorre o grupo simétrico $S y m_{n}$ e $(-1)^{\operatorname{sgn} \sigma}$ é igual a 1 , se $\sigma$ é uma permutação par, ou -1, se $\sigma$ é uma permutação ímpar. O polinômio $\left[x_{1}, \cdots, x_{n}\right]$ é conhecido como polinômio padrão de grau $n$ em Assoc $[X]$. Note que o polinômio padrão de grau 2 será simplesmente o comutador $\left[x_{1}, x_{2}\right]=x_{1} x_{2}-x_{2} x_{1}$. Neste sentido, a classe de álgebras que satisfaz um polinômio padrão é uma generalização da classe de álgebras comutativas.

O lema a seguir mostra que cada álgebra de dimensão finita é uma PI-álgebra.

Lema 2.4.2. Se A é uma $\Phi$-álgebra gerada por $n$ elementos, então A satisfaz a identidade $\left[x_{1}, x_{2}, \cdots, x_{n}\right]=0$. 
Essa demonstração poderá ser vista em [ZSSS82].

Em [ZSSS82], encontramos o seguinte resultado:

Lema 2.4.3. Toda álgebra algébrica de indice limitado é uma PI-álgebra.

Seja $u=x_{i_{1}}^{l_{1}} x_{i_{2}}^{l_{2}} \cdots x_{i_{h}}^{l_{h}}$, em que $i_{r} \neq i_{r+1}$, para $r \in\{1,2, \cdots, h-1\}$. Diremos que o número $h$ é a altura do monômio $u$.

Agora seja $A$ uma álgebra associativa gerada por um conjunto finito $\left\{a_{1}, a_{2}, \cdots, a_{k}\right\}$. Para cada polinômio $f=f\left(x_{1}, \cdots, x_{k}\right)$ da álgebra associativa livre Ass $\left[x_{1}, \cdots, x_{k}\right]$, iremos designar por $\bar{f}=f\left(a_{1}, \cdots, a_{k}\right)$ como a imagem do elemento $f$ pelo o homomorfismo da álgebra $A s s\left[x_{1}, \cdots, x_{k}\right] \rightarrow A$, que faz a aplicação $x_{i} \mapsto a_{i}$. Sejam $Y=\left\{f_{1}, f_{2}, \cdots, f_{l}\right\}$ um conjunto finito de polinômios homogêneos de $A s s\left[x_{1}, \cdots, x_{k}\right], \bar{Y}=\left\{\bar{f}_{1}, \bar{f}_{2}, \cdots, \bar{f}_{l}\right\}$ e $v$ um monômio de $A s s\left[x_{1}, \cdots, x_{k}\right]$. Vamos supor que existe um número natural $q$ e monômios associativos $u_{i}\left(x_{1}, \ldots, x_{l}\right)$, com o máximo de suas alturas igual a $q$, de modo que na álgebra $A$

$$
\bar{v}=\sum_{i} u_{i}\left(\bar{f}_{1}, \bar{f}_{2}, \cdots \bar{f}_{l}\right)
$$

e que também cada elemento $u_{i}\left(f_{1}, \ldots, f_{l}\right)$ tem o mesmo tipo ${ }^{5}$ de $v$. Nós chamamos o menor número $q$ com essa propriedade de altura do monômio $v$ com respeito ao conjunto $\bar{Y}$.

No caso em que as alturas em relação a $\bar{Y}$ de todas os monômios da álgebra $A$ são limitadas por algum número natural $h$, diremos que $A$ é uma álgebra de altura limitada (com respeito ao conjunto $\bar{Y}$ ) e que $h$ é a altura da álgebra $A$.

É sabido que toda PI-álgebra finitamente gerada é uma álgebra de altura limitada. Este fato tem o nome de teorema da altura.

Teorema 2.4.4 (Sobre a altura - Shirshov). Considere uma identidade polinomial admissivel de grau $n$ satisfeita no $\Phi$-álgebra associativa $A$ de um conjunto de geradores $\left\{a_{1}, \cdots, a_{k}\right\}$. Nessas condições, a álgebra $A$ tem altura limitada no que diz respeito ao conjunto $\bar{Y}$, em que $Y$ é o conjunto de todas as palavras de associativos Ass $\left[x_{1}, \cdots, x_{k}\right]$ de comprimento menor do que $n$.

Podemos inferir, em vistas desse teorema, os seguintes resultados:

Corolário 2.4.1. Toda PI-álgebra associativa tem altura limitada.

Corolário 2.4.2 (Kaplansky). Uma PI-álgebra algébrica, associativa e finitamente gerada sobre um $F$ é de dimensão finita.

Corolário 2.4.3 (Levitzki). Uma PI-álgebra associativa e finitamente gerada sobre um anel de operadores $\Phi$ em que cada elemento é nilpotente, é também nilpotente.

É interessante que se destaque que tais teoremas (de Kaplansky e de Levitzki, supracitados), pois foram obtidos por seus autores usando métodos completamente diferentes, mas podem ser apresentados como corolários de um único resultado. A generalidade destes teoremas torna-se ainda mais evidente após a consideração do conceito de finitude local. No que se segue fixamos um ideal $Z$ do anel $\Phi$.

\footnotetext{
${ }^{5}$ Consulte [ZSSS82] para saber mais sobre o tipo de um monômio.
} 
No Exemplo 2.1.10 apresentamos as álgebras de potências associativas. Definimos um elemento $a$ de uma $\Phi$-álgebra $A$ de potências associativas como sendo algébrico sobre $Z$ se existem elementos $z_{i} \in Z$ e um número natural $m$ tal que

$$
a^{m}=\sum_{i=1}^{m-1} z_{i} a^{i}
$$

Um corolário imediato do teorema sobre a altura é

Teorema 2.4.5. Se numa $\Phi$-álgebra associativa finitamente gerada $A$, com identidade polinomial admissivel de grau $n$, todos os produtos de geradores com menos de $n$ fatores são algébricos sobre $Z$, então a álgebra A é finita sobre $Z$.

Este teorema contém os teoremas de Kaplansky e Levitzki como casos particulares. Sua principal diferença deles é que a condição algébrica não é imposta a todos os elementos, mas apenas em um número finito deles. O seguinte lema é necessária para o estudo subsequente do conceito da finitude local.

Lema 2.4.6. Suponha que uma álgebra A é finita sobre $Z$. Nesse caso, A é localmente finita sobre $Z$. Ademais, se A uma álgebra finitamente gerada como $\Phi$-módulo, então qualquer subálgebra finitamente gerada $B$ de $A$ é também um $\Phi$-módulo finitamente gerado.

Em vistas do teorema 2.4.5 e do lema 2.4.6, temos o seguinte resultado:

Teorema 2.4.7. Se numa álgebra associativa A, com identidade polinomial admissivel de grau $n$, todos os produtos de geradores com menos de $n$ fatores são algébricos sobre $Z$, então a álgebra A é localmente finita sobre $Z$.

Falaremos mais sobre finitude e nilpotência local no capítulo, onde abordaremos alguns tópicos da teoria estrutural das álgebras de Jordan e de Lie.

Continuando este capítulo, vamos encerrar com a última sessão, sobre um tipo de variedade de grande importância neste estudo: as variedades não matriciais.

\subsection{Variedades não matriciais em Assoc}

Nesta seção, apresentaremos conceitos e resultados relativos às variedades não matriciais de álgebras associativas. Faz parte dessa tese avaliar quais tipos de generalizações são possíveis dentro de um ambiente não associativo. Portanto, nessa seção, variedade significará variedade de álgebras associativas.

Ao longo desta seção, $F$ é um corpo infinito e $F_{2}$ denota as matrizes $2 \times 2$ sobre $F$, isto é, $F_{2}=M_{2}(F)$.

Vamos ao conceito de variedade não matricial proposto por Latyshev.

Definição 1 (Latyshev). Diremos que uma variedade de $F$-álgebras associativas $\mathcal{M}$ é não matricial se $F_{2} \notin \mathcal{M}$.

Variedades não matriciais foram introduzidas por Latyshev, em [Lat77], e eram estudadas, inicialmente, no caso em que o corpo tinha característica zero. Álgebras geradas por elementos nil e sua conexão com variedades não matriciais foram estudadas por Billig, Riley e Tasic em [BRT97]. Variedades não matriciais foram caracterizadas por Chekanu, 
em [Cek79],como se segue: Uma variedade $\mathcal{M}$ é não matricial se, e somente se, qualquer álgebra em $\mathcal{M}$ gerada por um número finito de elementos algébricos é de dimensão finita. A lista a seguir é um exemplo mais amplo de equivalências com variedades não matriciais, que podem ser vistas em [MPR2011].

Teorema 2. Seja $\mathcal{M}$ uma variedade de álgebras associativas sobre um corpo $F$ infinito. As seguintes condições são equivalentes:

i) $F_{2} \notin \mathcal{M}$, ou seja, $\mathcal{M}$ é não matricial.

ii) Seja $A \in \mathcal{M}$ uma álgebra finitamente gerada. Para algum s, A satisfaz a identidade

$$
\left[x_{1}, x_{2}\right] \cdots\left[x_{2 s-1}, x_{2 s}\right] \equiv 0 .
$$

iii) Existe $m$ tal que qualquer álgebra em $\mathcal{M}$ satisfaz a identidade $[x, y]^{m} \equiv 0$.

iv) Para qualquer $A \in \mathcal{M}$, os elementos nilpotentes de $A$ formam um ideal.

v) Seja $A \in \mathcal{M}$. Qualquer conjunto finito $\left\{a_{1}, a_{2}, \cdots a_{k}\right\}$ de elementos algébricos em $A$ gera uma álgebra de dimensão finita $A_{0} \subseteq A$.

vi) Se $A \in \mathcal{M}$ e $a, b \in A$ são elementos nilpotentes, então $a+b$ também é nilpotente.

vii) Se $A \in \mathcal{M}$ e $a \in A$ é nilpotente, então o ideal (a) é nilpotente.

viii) Se $A \in \mathcal{M}$ e $a \in A$ é nilpotente, então o ideal à esquerda Aa (ou o ideal à direita aA) é nilpotente.

Recomendamos ao leitor a avaliação deste resultado, em [MPR2011], a fim de comparar técnicas usadas para álgebras associativas com as técnicas nas classes de álgebras (não associativas) a serem apresentadas nos próximos capítulos.

Outro resultado, devido a Kemer, em [Kem80], apresenta identidades em variedades não matriciais (sobre um corpo de característica zero).

Teorema 2.5.1 (Kemer). As álgebras de uma variedade não matricial arbitrária (de álgebras associativas) satisfazem a seguinte identidade, para algum natural $r$ :

$$
\left[\left[x_{1}, y_{1}\right],\left[z_{1}, t_{1}\right], u_{1}\right] \cdot \ldots \cdot\left[\left[x_{r}, y_{r}\right],\left[z_{r}, t_{r}\right], u_{r}\right]=0
$$

Ainda em [Kem80], ele se utiliza da álgebra de Grassmann (sobre um conjunto enumerável de geradores) para estabelecer o seguinte:

Teorema 2.5.2 (Kemer). Se $\mathcal{M}$ é uma variedade não matricial e $G \otimes G \notin \mathcal{M}$, em que $G$ é a álgebra de Grassmann, então $\mathcal{M}$ satisfaz a seguinte identidade, para algum natural $r$ :

$$
\left[x_{1}, y_{1}, z_{1}\right] \cdot \ldots \cdot\left[x_{r}, y_{r}, z_{r}\right]=0
$$

Na expressão acima, $[x, y, z]$ é o comutador duplo, isto é, $[x, y, z]=[[x, y], z]$.

Nosso objetivo é coletar diferentes caracterizações de variedades não matriciais para álgebras associativas e adaptar algumas demonstrações em algumas classes de álgebras não associativas: as álgebras de Jordan, as álgebras alternativas, as (-1,1)-álgebras e as álgebras de Jordan não comutativas. 


\section{Capítulo 3}

\section{Álgebras de Jordan}

Recordemos a definição de álgebras de Jordan.

Definição 3 (Álgebra de Jordan). Uma álgebra $J$ é dita ser uma álgebra de Jordan se ela satisfaz as seguintes identidades:

$$
\left(x^{2}, y, x\right)=0 \quad(\text { Jordan })
$$

$\mathrm{e}$

$$
[x, y]=0 \quad \text { (Comutativa) }
$$

Note que a identidade de Jordan indica que álgebras de Jordan podem ser não associativas. A identidade comutativa para álgebras diz que álgebras de Jordan são comutativas.

Álgebras de Jordan foram introduzidas em 1932, por Pascual Jordan, como uma tentativa de estabelecer um programa que visava interpretar fenômenos da Mecânica através de um novo sistema algébrico.

Resumidamente, certos fenômenos físicos tratados como "observáveis" estavam fortemente relacionados com operadores simétricos (matrizes hermitianas). Ao impor a condição da comutatividade (lembremos que a álgebra de matrizes sobre um corpo $F, M_{n}(F)$, não é comutativa) para uma multiplicação especial sobre estas matrizes, perdia-se a associatividade desta multiplicação.

Se $A$ e $B$ são duas matrizes $n \times n, n$ é um natural fixado, sobre o conjunto dos reais $\mathbb{R}$, por exemplo, é sabido, de forma geral, que $A B \neq B A$, para a multiplicação usual definida sobre tais matrizes. Todavia, se definimos a multiplicação o

$$
A \circ B=\frac{1}{2}(A B+B A)
$$

então $A \circ B=B \circ A$. Entretanto, a operação $\circ$ não é associativa, isto é, é possível escolher três matrizes $A, B$ e $C, n \times n$, tais que

$$
(A \circ B) \circ C \neq A \circ(B \circ C) .
$$

Apesar de ser não associativa, a multiplicação o satisfaz a identidade

$$
((A \circ A) \circ B) \circ A=(A \circ A) \circ(B \circ A) .
$$

Como generalização da construção acima, seja $(\mathbf{A},+, \cdot)$ uma álgebra associativa e 
defina a operação •, a partir da multiplicação ·, da seguinte forma:

$$
a \bullet b=a \cdot b+b \cdot a, \quad a, b \in \mathbf{A} .
$$

Dessa forma, $(A,+, \bullet)$ é uma álgebra que, para quaisquer elementos $a$ e $b$, satisfaz as identidades

$$
\begin{aligned}
a \bullet b & =b \bullet a \\
\left(a^{2} \bullet b\right) \bullet a & =a^{2} \bullet(b \bullet a) .
\end{aligned}
$$

As identidades acima descritas motivaram a inserção das estruturas de Jordan como objeto matemático. Este tema de estudo prosperou dentro da Matemática (e também na própria Física) e hoje conta com uma teoria bem desenvolvida e largamente estudada pela comunidade matemática (principalmente na Álgebra).

Mais uma vez, aqui denotamos por $\Phi$ um anel de escalares (associativo, comutativo e unitário) para qual consideraremos $\Phi$-álgebras. Se $(\mathbf{A},+, \cdot)$ é uma $\Phi$-álgebra e $a \in \mathbf{A}$ é um elemento arbitrário, observe que

$$
a \bullet a=a^{2}+a^{2}=2 a^{2} .
$$

Logo, se $\Phi$ for um anel de característica 2, a operação será trivializada: $a \bullet a=0$, mesmo que $a^{2} \neq 0$.

Com tais observações, é desejável que $\Phi$ seja um anel cuja característica é diferente de 2. Também, é desejável que em um elemento arbitrário $a \in \mathbf{A}$, suas potências por · e - coincidam. Dessa forma, "readaptaremos" a operação "•" através da seguinte condição: $\Phi$ é um anel de escalares cuja característica é diferente de 2 e tal que o elemento " $1+1=$ 2" seja inversível. Desta forma, estabelecemos a operação o da seguinte forma:

$$
a \circ b=\frac{1}{2}(a b+b a)
$$

Note que

$$
a \circ a=\frac{1}{2}(a \cdot a+a \cdot a)=\frac{1}{2}(2 a \cdot a)=a \cdot a .
$$

Por indução, pode-se estabelecer que as potências de $a$ coincidem, seja através de "o", seja através de ".".

Neste capítulo, consideraremos o anel de escalares nestas observações: $\Phi$ é associativo, comutativo, unitário, char $\Phi \neq 2$ e 2 é inversível em $\Phi$.

A multiplicação o definida aqui é conhecida como multiplicação de Jordan ou produto de Jordan e será fundamental para auxiliar no nosso programa de estudos. A álgebra $(\mathbf{A},+, \circ)$ é conhecida como álgebra adjunta de $\mathbf{A}$ e será denotada por $\mathbf{A}^{(+)}$. Observe que $\mathbf{A}$ e $\mathbf{A}^{(+)}$coincidem enquanto conjuntos, mas se diferenciam pela multiplicação. Ademais, se $(\mathbf{A},+, \cdot)$ é uma álgebra associativa, é fato bastante conhecido que $\mathbf{A}^{(+)}$é álgebra de Jordan (de acordo com a Definição 3 ). Se $(\mathbf{A},+, \cdot)$ não for associativa, não há garantias de que $\mathbf{A}^{(+)}$seja uma álgebra de Jordan: apenas pode-se garantir que $\mathbf{A}^{(+)}$é uma álgebra comutativa. Note também que, se $(\mathbf{A},+, \cdot)$ é uma álgebra anticomutativa (Exemplo 2.1.8), então $\mathbf{A}^{(+)}$é uma zero álgebra (Exemplo 2.1.5). 


\subsection{Definições e exemplos}

Ao longo desta seção, $F$ denota um corpo de característica diferente de 2 .

Dizemos que uma álgebra de Jordan $(J,+, \cdot)$ é especial se ela pode ser injetada numa álgebra associativa $\left(\mathbf{A}^{+},+, \cdot\right)$, isto é, tendo em mente a multiplicação de Jordan "o", $J$ é especial se existe $B \subseteq \mathbf{A}$ subálgebra tal que $J=B^{(+)}$. Caso contrário, diremos que a álgebra de Jordan é excepcional.

Relembremos que a variedade das álgebras de Jordan será denotada por Jord.

Recordemos a definição de elementos hermitianos.

Definição 3.1.1 (Involução). Seja $A$ uma $\Phi$-álgebra. Um $\Phi$-endomorfismo $*$ de $A$, em que, para $a \in A, *: a \mapsto a^{*}$, é dito ser uma involução se:

I1) $\left(a^{*}\right)^{*}=a$;

I2) $(a b)^{*}=b^{*} a^{*}$.

Definição 3.1.2 (Elementos Hermitianos). Seja $(A, *)$ uma álgebra com involução *. Um elemento $a \in A$ é dito ser hermitiano se $a^{*}=a$. O conjunto dos elementos hermitianos de $A$ será denotado por $\mathcal{H}(A, *)$.

Seja $M_{n}(A)$ a álgebra de matrizes quadradas de ordem $n$ sobre uma álgebra $A$. Se $(A,-)$ é uma álgebra com involução "-", em $M_{n}(A)$ definiremos a involução padrão a partir da transposição, isto é, se $\left(m_{i j}\right) \in M_{n}(A)$, então $\left(m_{i j}\right)^{*}=\left(\overline{m_{j i}}\right)$. Se $A$ for uma álgebra comutativa e nenhuma involução for previamente definida em $A$, consideraremos em $M_{n}(A)$ a involução trivial: a transposição de matrizes.

Dessa forma, se $F$ é um corpo arbitrário, a involução padrão a ser considerada em $M_{n}(F)$ é a transposição de matrizes, isto é, $t: M_{n}(F) \rightarrow M_{n}(F)$ definida por $\left(m_{i j}\right)^{t}=$ $\left(m_{j i}\right)$.

É claro que a transposição não é o único tipo de involução a ser definida em $M_{n}(F)$, como veremos adiante.

Pela definição anterior, concluiremos que, dado um corpo $F$ arbitrário e se não houver qualquer especificação com respeito à transposição $*$ sobre $M_{n}(F)$, os elementos hermitianos em $M_{n}(F)$ serão as matrizes simétricas.

Munido do produto usual de matrizes, o conjunto $\mathcal{H}_{n}=\mathcal{H}\left(M_{n}(F), t\right)$ não é uma álgebra, pois o produto usual de matrizes não é fechado em $\mathcal{H}_{n}$. Consideremos o produto de Jordan "o" definido sobre esse conjunto, isto é,

$$
A \circ B=\frac{1}{2}(A B+B A), \quad A, B \in \mathcal{H}_{n}=\mathcal{H}\left(M_{n}(F) ; t\right) .
$$

Dessa forma, $A \circ B \in \mathcal{H}_{n}$, se $A, B \in \mathcal{H}_{n}$. Portanto, $\left(\mathcal{H}_{n},+, \circ\right) \subseteq M_{n}(F)^{(+)}$é uma álgebra de Jordan especial.

Consideremos alguns exemplos de álgebras de Jordan.

Exemplo 3.1.3 (Tipo Hermitiano). Sejam $(A, *)$ uma álgebra associativa sobre $\Phi$ com involução $*$ e $J=\mathcal{H}(A, *)$ munida do produto de Jordan definido a partir de $A$, isto é, se $x, y \in \mathcal{H}(A, *)$, então $x \circ y=\frac{1}{2}(x y+y x)$. Tal como no caso matricial, $\mathcal{H}(A, *)$ é uma álgebra de Jordan especial.

Ademais, suponha que $(A, *)$ seja uma álgebra (associativa) *-simples, isto é, os únicos ideais $*$-invariantes são os triviais. Nesse caso, a álgebra $J=\mathcal{H}(A, *)$ é conhecida como álgebra de Jordan do tipo Hermitiano. 
Exemplo 3.1.4 (Tipo Clifford). Tipo Clifford: Sejam $V$ um espaço vetorial de dimensão maior que 1 sobre um corpo $F$ de característica diferente de 2 e $f$ uma forma bilinear simétrica não degenerada definida sobre $V$. Denote por $J=J(V, f)=V \dot{+} F \cdot 1$ a álgebra definida a partir de $V$ e $f$, cuja multiplicação entre os vetores de $V$ é definida por $v \cdot w=$ $f(v, w) 1$, ou seja,

$$
(v+\alpha 1)(w+\beta 1)=(\alpha w+\beta v)+\alpha \beta+f(v, w) 1 .
$$

A álgebra $J(V, f)$ é uma álgebra de Jordan especial, conhecida como álgebra de Jordan do tipo Clifford.

Se $f$ é não degenerada e $\operatorname{dim} V>1$, então $J(V, f)$ é simples.

Relembremos que uma forma bilinear é dita ser não degenerada se dado a tal que $f(a, x)=0$, para todo $x \in A$, podemos concluir que $a=0$.

Exemplo 3.1.5 (Tipo Albert). Considere a álgebra de Albert $\mathcal{H}\left(M_{3}(\mathbb{O}), *\right)$, em que $\mathbb{O}$ é álgebra de octônios ${ }^{1}$ (sobre $F$ ) e * é a involução induzida por $\mathbb{O}$. A álgebra $\mathcal{H}\left(M_{3}(\mathbb{O}), *\right.$ ) tem dimensão vetorial (sobre $F$ ) igual a 27. Agora seja $J$ uma álgebra de Jordan que satisfaz a relação

$$
\bar{F} \otimes_{F} J \cong \mathcal{H}\left(M_{3}(\mathbb{O}), *\right),
$$

em que $\bar{F}$ é o fecho algébrico de $F$. Tal álgebra é conhecida como álgebra de Jordan do tipo Albert.

Já é sabido que uma álgebra de Jordan do tipo Albert não é especial, isto é, ela é excepcional.

O conjunto $\mathcal{H}\left(M_{3}(\mathbb{O}), *\right)$ é precisamente:

$$
\mathcal{H}\left(M_{3}(\mathbb{O}), *\right)=\left\{\left[\begin{array}{lll}
\alpha & a & b \\
a & \beta & c \\
b & c & \gamma
\end{array}\right], \quad \alpha, \beta, \gamma \in F, \quad a, b, c \in \mathbb{O}\right\} .
$$

Portanto, $\operatorname{dim} \mathcal{H}\left(M_{3}(\mathbb{O}), *\right)=27$.

Observação 3.1.6. A álgebra de elementos hermitianos $\mathcal{H}\left(M_{2}(\mathbb{O}), *\right)$ é uma álgebra de Jordan especial, mas $H\left(M_{4}(\mathbb{O})\right.$, *) não é uma álgebra de Jordan.

É fato bastante conhecido, que pode ser verificado em [McCr04] e [ZSSS82], que as álgebras do tipo Clifford, Hermitiano e Albert são álgebras de Jordan simples.

Consideremos agora algumas identidades polinomiais (não triviais) em Jord.

\subsubsection{PI-álgebras de Jordan}

De forma análoga às álgebras associativas, conforme a seção 2.4, definimos Jord, a variedade das álgebras de Jordan, através das identidades $f_{1}=[x, y]$ e $f_{2}=\left(x^{2}, y, x\right)$.

Conforme observado no início do capítulo, Jord será construída supondo que seus elementos são $\Phi$-álgebras, em que $\Phi$ é um anel de escalares já especificado para o caso de álgebras de Jordan. Seja Jord $[X]$ a álgebra livre de Jordan sobre um conjunto de variáveis enumerável $X$. Seja Assoc $[X]$ a álgebra livre associativa sobre $X$. Defina a álgebra livre de Jordan especial $S \operatorname{Jord}[X]$ de forma natural, isto é, $S \operatorname{Jord}[X]=\operatorname{Assoc}[X]^{(+)}$.

\footnotetext{
${ }^{1}$ Consulte [ZSSS82] para maiores detalhes.
} 
Dizemos que um elemento $f \in \operatorname{Jord}[X]$ é essencial se a imagem $\bar{f}$ com respeito ao homomorfismo natural $\operatorname{Jord}[X]$ sobre $S \operatorname{Jord}[X]$ é um elemento admissível quando considerado como um elemento da álgebra associativa livre Assoc $[X]$.

Exemplo 3.1.7. Considere em Jord $[X]$ o polinômio $f\left(x_{1}, x_{2}, x_{3}\right)=4\left(x_{1}, x_{2}, x_{3}\right)$. O polinômio $f$ é essencial em $\operatorname{Jord}[X]$, pois

$$
\begin{aligned}
\overline{f\left(x_{1}, x_{2}, x_{3}\right)} & =4\left[\left(x_{1} \circ x_{2}\right) \circ x_{3}-x_{1} \circ\left(x_{2} \circ x_{3}\right)\right] \\
& =x_{2}\left(x_{1} x_{3}\right)+\left(x_{3} x_{1}\right) x_{2}-\left(x_{1} x_{3}\right) x_{2}-x_{2}\left(x_{3} x_{1}\right) \\
& =\left[x_{2}, x_{1} x_{3}\right]-\left[x_{2}, x_{3} x_{1}\right] \\
& =\left[x_{2},\left[x_{1}, x_{3}\right]\right] .
\end{aligned}
$$

Em outras palavras, $\bar{f}=\left[x_{2},\left[x_{1}, x_{3}\right]\right]$ é um polinômio admissível em Assoc $[X]$.

Dizemos que uma álgebra de Jordan $J$ satisfaz uma identidade polinomial essencial se existe um elemento essencial $f\left(x_{1}, \cdots, x_{n}\right) \in \operatorname{Jord}[X]$ tal que $f\left(a_{1}, \cdots, a_{n}\right)=0$, para quaisquer $a_{1}, \cdots, a_{n} \in J$. Uma PI-álgebra de Jordan, portanto, é uma álgebra de Jordan que satisfaz uma identidade polinomial essencial $f$.

Exemplo 3.1.8. Qualquer álgebra associativa e comutativa é uma PI-álgebra de Jordan. Primeiro, note que qualquer álgebra associativa satisfaz a identidade $f_{1}=4\left(x_{1}, x_{2}, x_{3}\right)$. Por causa do Exemplo 3.1.7, $f_{1}$ é admissível em Jord $[X]$. Ademais, qualquer álgebra comutativa satisfaz a identidade $f_{2}=\left[x_{2},\left[x_{1}, x_{3}\right]\right]$.

Exemplo 3.1.9. A álgebra de Jordan $M_{2}(\Phi)^{(+)}$satisfaz o polinômio essencial $f(x, y, z, r, s)=$ $128\left((x, y, z)^{2}, r, s\right)$.

Exemplo 3.1.10. Uma nil-álgebra de Jordan de índice finito $n$ satisfaz o polinômio essencial $f(x)=x^{n}$.

Note que $x^{n}$ está bem definida se $x$ está em uma álgebra de Jordan $J$, pois álgebras de Jordan são álgebras de potências associativas. Este fato nos será útil mais adiante.

No caso de Jordan, é razoável estabelecer uma conexão entre a álgebras livre Assoc $[X]$ e Assoc $[X]^{(+)}$, pois Assoc $[X]$ é associativa e Assoc $[X]^{(+)}$é de Jordan. De forma geral, em uma variedade arbitrária de álgebras $\mathcal{V}$, diremos que uma álgebra é PI se ela satisfizer uma identidade polinomial essencial não trivial em $\mathcal{V}$.

$\mathrm{Na}$ próxima seção, introduziremos alguns radicais em Jord.

\subsection{Radicais em Jord}

Uma álgebra tem uma propriedade local se toda subálgebra finitamente gerada tem essa propriedade. Dessa forma, introduzimos o conceito de nilpotência local. Relembremos que boa parte desse estudo já foi feita no capítulo anterior.

Um ideal localmente nilpotente $\operatorname{Locnil}(A)$ tal que a álgebra quociente $A / \operatorname{Locnil}(A)$ não contêm ideais não nulos localmente nilpotentes é chamado de radical localmente nilpotente da álgebra $A$. Como a imagem homomórfica de uma álgebra localmente nilpotente é uma álgebra localmente nilpotente, o ideal $\operatorname{Locnil}(A)$ contém qualquer outro ideal localmente nilpotente da álgebra $A$.

Apresentaremos alguns resultados com o objetivo de mostrar que em toda álgebra de Jordan o radical localmente nilpotente existe. O caminho aqui adotado será um pouco diferente do mostrado na subseção 2.3.1. 
Lema 3.2.1. Seja $J$ uma álgebra de Jordan finitamente gerada. Os ideais $J^{[k]}$ de $J$ também são álgebras finitamente geradas, em que $J^{[1]}=J^{3}$ e $J^{[k+1]}=\left(J^{[k]}\right)^{3}$.

Lema 3.2.2. Se tanto o ideal I de uma álgebra de Jordan $J$ e a álgebra quociente $J / I$ são localmente nilpotentes, então a álgebra J também é localmente nilpotente.

As demonstrações dos lemas 3.2.1 e 3.2.2 podem ser vistas em [ZSSS82].

Teorema 3.2.3. Toda álgebra de Jordan tem um radical localmente nilpotente.

Fixada arbitrariamente uma álgebra de Jordan $J$, consideremos a soma Locnil $(J)$ de todos os locais ideais nilpotentes. Se $L_{1}$ e $L_{2}$ são dois ideais localmente nilpotentes, então, pelo teorema do homorfismo,

$$
\frac{L_{1}+L_{2}}{L_{1}} \cong \frac{L_{2}}{L_{1} \cap L_{2}}
$$

Por causa do Lema 3.2.2, segue-se que $L_{1}+L_{2}$ também é um ideal localmente nilpotente. Por indução finita, é fácil ver que a soma de um número finito de ideais localmente nilpotentes é um ideal localmente nilpotente. Sejam $l_{l}, l_{2}, \cdots, l_{n}$, um número finito de elementos de Locnil $(J)$. Todos estes elementos encontram-se na soma de um número finito de ideais localmente nilpotentes e, portanto, geram uma subálgebra nilpotente. Conseqüentemente, o ideal Locnil $(J)$ é localmente nilpotente. Em vista do Lema 3.2.2, a álgebra quociente $J /$ Locnil $(J)$ não contém nulos ideais localmente nilpotentes, pois, se assim não o fosse, haveria um ideal localmente nilpotente $L_{\alpha}$ tal que $L_{\alpha} \nsubseteq$ Locnil $(J)$, o que contraria a definição de Locnil $(J)$. Isto significa que Locnil $(J)$ é o radical localmente nilpotente da álgebra $J$, o que prova o teorema.

Na Subseção 2.3.1, já havia sido comentada a existência do radical localmente nilpotente para álgebras de Jordan. Apresentamos esta construção (que culmina com o Teorema 3.2.3) como um caminho alternativo ao radical Locnil já apresentado na subseção citada.

Exibiremos a construção de outro radical para álgebras de Jordan.

Sejam $J$ uma álgebra de Jordan e $a \in J$. Defina o seguinte operador:

$$
U_{a}:=2 R_{a}^{2}-R_{a^{2}}
$$

Lembremos que as operações $L_{a}$ e $R_{a}$ (multiplicação à esquerda e à direita, respectivamente) já foram definidas no Capítulo 2. Mantendo a mesma notação, dado $x \in J$, o operador $U_{a}$ funciona da seguinte forma:

$$
x U_{a}=2 x R_{a}^{2}-x R_{a^{2}}=2(x a) a-x \cdot a^{2} .
$$

Um elemento $z$ de uma álgebra de Jordan $J$ é chamado de divisor de zero absoluto se $J U_{z}=(0)$, isto é, se $U_{z}$ é o operador zero. Se o único divisor de zero absoluto em $J$ é o elemento 0, a álgebra $J$ é chamada de não degenerada.

Uma outra forma (equivalente, obviamente) de entender os divisores de zero absolutos será mostrada a seguir.

Se $J$ é uma álgebra de Jordan, definimos a tripla de Jordan:

$$
\{a, b, a\}=2(b \cdot a) \cdot a-b \cdot a^{2} .
$$


Um elemento $a \in J$ é dito ser um divisor de zero absoluto se $\{a, J, a\}=(0)$. Esta definição concorda com o caso associativo ${ }^{2}$, já que, para uma álgebra associativa $\mathbf{A}$, um elemento $b$ é um divisor de zero absoluto se, e somente se, o é em $\mathbf{A}^{(+)}$.

Vamos denotar por $\mathcal{M}(J)$ o ideal da álgebra de Jordan $J$ gerado por todos os seus divisores de zero absolutos. A álgebra $J$ é dita ser não degenerada, portanto, se $\mathcal{M}(J)=$ $(0)$.

Seja $I$ um ideal trivial da álgebra $J$. Para quaisquer elementos $x \in J$ e $z \in I$ temos

$$
x U_{z}=2(x z) z-x z^{2}=0 .
$$

A equação acima nos diz que cada elemento de um ideal trivial $I$ é um divisor de zero absoluto da álgebra $J$, em particular, $I \subseteq \mathcal{M}(J)$. Consequentemente, a não degeneração de uma álgebra de Jordan $J$ implica $J$ é semiprima ${ }^{3}$. Se a recíproca é verdadeiro, ainda é desconhecido. Também é desconhecido quando o ideal $\mathcal{M}(J)$ é localmente nilpotente. Sabe-se que apenas $\mathcal{M}(J)$ é um nil ideal.

A classe de álgebras de Jordan não degeneradas é, portanto, uma subclasse da classe de álgebras de Jordan semiprimas. Álgebras de Jordan são degeneradas são a classe semisimples de um determinado radical.

Lema 3.2.4. Sejam J uma álgebra de Jordan e $J^{\#}$ a álgebra obtida pelo adjunto formal a $J$ de um elemento de identidade 1. Se z é um divisor de zero absoluto em $J$ e $x \in J^{\#}$, $z U_{x}$ também é um divisor de zero absoluto em $J$.

Lema 3.2.5. O ideal $\mathcal{M}(J)$ é gerado como $\Phi$-módulo por divisores de zero absolutos.

Defina $\operatorname{Degen}_{1}(J)=\mathcal{M}(J)$. A partir daí, dado um ordinal $\beta$, defina Degen ${ }_{\beta}$ a partir de todos os ordinais $\alpha$ menores que $\beta$.

Se $\beta$ é um ordinal limite, definimos

$$
\operatorname{Degen}_{\beta}(J)=\cup_{\alpha<\beta} \operatorname{Degen}_{\alpha}(J) .
$$

Se $\beta$ não é um ordinal limite, então existe o ordinal $\beta-1$ e definimos $\operatorname{Degen}_{\beta}(J)$ como o ideal tal que

$$
\operatorname{Degen}_{\beta}(A) / \operatorname{Degen}_{\beta-1}(A)=\mathcal{M}\left(J / \operatorname{Degen}_{\beta-1}(A)\right) .
$$

A cadeia ascendente de ideais

$$
\operatorname{Degen}_{1}(J) \subseteq \operatorname{Degen}_{2}(J) \subseteq \cdots \subseteq \operatorname{Degen}_{\alpha}(J) \subseteq \cdots
$$

estabiliza, para algum oridnal $\gamma$. Defina

$$
\operatorname{Degen}(J)=\operatorname{Degen}_{\gamma}(J) \text {. }
$$

\footnotetext{
${ }^{2} \mathrm{Na}$ classe das álgebras alternativas, um elemento a de uma álgebra alternativa $\mathbf{A}$ é dito ser um divisor de zero absoluto se $a A a=(0)$. A definição de divisor de zero absoluto no caso associativo segue da mesma forma, de imediato, pois toda álgebra associativa é alternativa. Na verdade, a definição de divisor de zero absoluto surge para o caso associativo e sua posterior generalização ao caso alternativo se mostra bastante razoável.

${ }^{3}$ Uma álgebra A é dita ser semiprima se não possui ideais triviais não nulos. Por sua vez, um ideal $I \unlhd \mathbf{A}$ é trivial se, com a multiplicação proveniente de A, I é uma zero álgebra (Definição 2.1.5), isto é, se $I^{2}=(0)$.
} 
Lema 3.2.6. A álgebra quociente $J / \operatorname{Degen}(J)$ é não degenerada e Degen $(J)$ é o menor ideal com essa propriedade.

Observação 3.2.1. A construção deste radical é feito de forma análoga ao radical de Baer (consulte [ZSSS82]).

Corolário 3.2.2. Uma álgebra de Jordan $J \neq(0)$ não pode ser aplicada homomorficamente sobre uma álgebra não degenerada se, e somente se, $J=\operatorname{Degen}(J)$.

Vamos denotar por Degen classe de álgebras de Jordan $J$ em que $J=\operatorname{Degen}(J)$.

Lema 3.2.7. Sejam I um ideal de uma álgebra de Jordan $J$ e $x$ um divisor de zero absoluto da álgebra I. Dessa forma, J tem também um divisor de zero absoluto contido em I.

Por fim, temos o seguinte resultado:

Teorema 3.2.8. A classe Degen é radical.

Todos estes resultados podem ser consultados em [ZSSS82].

Tendo em vista o Teorema 2.3.3, a propriedade $R 1$ ) é imediata para Degen. Provaremos a propriedade $R 4$ ). Se a álgebra $J$ é tal que existe um Degen-ideal não nulo em qualquer imagem homomórfica de $J$, pelo Lema 3.2.7, existe um divisor de zero absoluto em qualquer imagem homomórfica de $J$, ou seja, a álgebra $J$ não pode ser mapeada homomorficamente em uma álgebra não degenerada. Isso significa que $J \in$ Degen, o que prova a condição $R 4$ ) para a classe Degen.

O radical Degen, construído nestes últimos resultados, é chamado de radical de $M c$ Crimmon ou radical degenerado.

Desta forma, podemos definir, com base nos resultados apresentados nesta seção e na subseção 2.3.1 os seguintes radicais na classe das álgebras de Jordan:

- $\mathrm{Nil}(\mathbf{A})$, o nil radical de A, também conhecido como nil radical superior ou radical de Köethe.

- Locnil(A), o radical localmente nilpotente de A, também conhecido como radical de Levitzki.

- $\mathcal{L}_{Z}(\mathrm{~A})$, o radical localmente finito (sobre um certo ideal $Z$ do anel de escalares de A), que induz a existência de um radical de dimensão finita local.

- Degen(A), o radical não degenerado de A, também conhecido como radical de McCrimmon.

A respeito dos radicais acima mencionados, nos interessa saber localizá-los no reticulado dos radicais. 
Teorema 3.2.9 (McCrimmon, Zel'manov). Em qualquer álgebra de Jordan J as inclusões abaixo são válidas:

$$
\operatorname{Degen}(J) \subseteq \operatorname{Locnil}(J) \subseteq \operatorname{Nil}(J)
$$

De fato, McCrimmon mostrou que $\operatorname{Degen}(J) \subseteq \operatorname{Nil}(J)$ ([McCr04]). Posteriormente, Zel'manov demonstrou que $\operatorname{Degen}(J) \subseteq \operatorname{Locnil}(J)$ ([Zel79]).

Com base neste resultado, podemos reescrever o Teorema 2.3.1.18 da seguinte forma:

Teorema 3.2.3. Se J é uma álgebra de Jordan, então $J / \operatorname{Nil}(J)$ é isomorfa a uma soma subdireta de álgebras de Jordan primas não degeneradas.

Demonstração.

$J / \operatorname{Nil}(J) \hookrightarrow \prod J_{\alpha}$, pois $J_{\alpha}$ é prima não degenerada, pois $\operatorname{Nil}\left(J_{\alpha}\right)=(0), \forall \alpha$.

Outro resultado, devido a Zel'manov, será importante ao nosso estudo:

Teorema 3.2.10 (Zel'manov, [Zel78]). Se uma álgebra de Jordan J sobre um corpo satisfaz uma identidade polinomial, então

$$
\operatorname{Nil}(J)=\operatorname{Locnil}(J)=\operatorname{Degen}(J) .
$$

Em particular, se $J$ é nil de indice finito, então é localmente nilpotente: $J=\operatorname{Locnil}(J)$.

Em [Med88-2], o seguinte resultado é estabelecido:

Teorema 3.2.4 (Medvedev). Se J é uma PI-álgebra de Jordan finitamente gerada, então $\operatorname{Nil}(J)$ é nilpotente.

Em [McCr04] e [ZSSS82] podemos verificar que as álgebras do tipo Clifford, Hermitiano e Albert (reveja os exemplos 3.1.5, 3.1.4 e 3.1.3) são álgebras de Jordan simples. Surge uma questão natural: há outras álgebras de Jordan simples além dessas três?

A resposta a essa pergunta é negativa e foi dada por Efim Zel'manov, em 1983, no seu teorema de classificação de álgebras de Jordan simples.

Teorema 3.2.5 (Zel'manov, [Zel83]). As álgebras de Jordan simples são precisamente classificadas em um dos tipos abaixo:
1. Tipo Clifford;
2. Tipo Hermitiano;
3. Tipo Albert.

Teorema 3.2.6 (Zel'manov, [Zel83]). As álgebras de Jordan primas não degeneradas são precisamente:

$\diamond$ Formas Hermitianas: $H(B, *) \triangleleft J \subseteq H(Q(B), *)$, para uma álgebra associativa $*$ prima $B$ com involução * e seu anel de Martindale 4 de quocientes simétricos $Q(B)$;

\footnotetext{
${ }^{4}$ Consulte [McCr04].
} 
$\diamond$ Formas de Clifford: álgebras de Jordan especiais cujo fecho central é isomorfo a uma álgebra de Jordan simples do Tipo Clifford;

$\diamond$ Formas de Albert: álgebras de Jordan excepcionais cujo fecho central é isomorfo a uma álgebra de Jordan simples do Tipo Albert.

Ainda, este importante resultado é válido para PI-álgebras de Jordan.

Teorema 3.2.7 (Zel'manov). Cada ideal não nulo de uma PI-álgebra de Jordan não degenerada tem intersecção não nula com o centro (por isso, se o centro é um corpo, a álgebra é simples). O fecho central de uma PI-álgebra não degenerada e prima é central simples. Qualquer PI-álgebra de Jordan primitiva é simples. Cada PI-álgebra de Jordan simples ou é de dimensão finita ou é do tipo Clifford.

\subsection{Variedades não matriciais em Jord}

Voltamos ao tema de variedade não matriciais. Seja $\mathcal{V}$ uma variedade de álgebras de Jordan, sobre um corpo $F$ de característica diferente de 2, que não contém álgebras de Jordan de tipo matricial. No caso associativo, uma variedade é não matricial se ela não contém $F_{2}$, as matrizes quadradas de ordem 2 sobre $F$ (Definição 1 ).

De agora em diante, em todo o trabalho, denotaremos, por simplificação de notação, as matrizes quadradas $2 \times 2$ sobre $F$ por $F_{2}$ (ao invés de usar $M_{2}(F)$ ).

A álgebra de matrizes $F_{2}$, munida da multiplicação usual de matrizes, não é uma álgebra de Jordan. Uma álgebra de Jordan matricial similar a $F_{2}$ é a álgebra $F_{2}{ }^{(+)}$, todavia ela não se constitui um "tipo matricial minimal", pois $\mathcal{H}_{2} \subseteq F_{2}{ }^{(+)}$, munida das operações "+" e "o", é uma álgebra de Jordan. A álgebra $\mathcal{H}_{2}$, conforme já mencionado, é a álgebra de elementos hermitianos (simétricos) de $F_{2}$ através da transposição $t$ :

$$
\mathcal{H}_{2}=\mathcal{H}\left(F_{2}, t\right)
$$

Como no caso associativo, uma definição similar para variedades de álgebras de Jordan consiste em remover uma álgebra simples minimal cuja dimensão seja maior que 1. No caso associativo, $F_{2}$ é uma álgebra minimal satisfazendo esta condição. No caso de álgebras de Jordan, a álgebra simples minimal de dimensão maior que 1 é $\mathcal{H}_{2}$, a álgebra das matrizes hermitianas (simétricas) $2 \times 2$ sobre $F$.

Tais observações nos motivam à seguinte definição:

Definição 4. Uma variedade $\mathcal{V}$ de álgebras de Jordan é dita ser não matricial se $\mathcal{H}_{2} \notin \mathcal{V}$.

Nosso trabalho para álgebras de Jordan consistirá na utilização dos teoremas de classificação de Zel'manov para determinar resultados em variedade não matriciais.

O próximo resultado, estabelecido de maneira original nesta tese, oferece uma critério que relaciona "estrutura de álgebras de Jordan" e "variedade não matriciais".

Proposição 3.3.1. Seja $\mathcal{M}$ uma variedade não matricial de álgebras de Jordan sobre um corpo infinito $F$ de característica diferente de 2. Se J é uma álgebra de Jordan prima não degenerada em $\mathcal{M}$, então $J$ é associativa.

Demonstração. Seja $J$ uma álgebra de Jordan prima não degenerada em $\mathcal{M}$. Note que $J$ está em uma variedade não matricial (que é definida por certas identidades). Logo, $J$ é PIálgebra prima e não degenerada. Pelo Teorema 3.2.7, o fecho central de $J, Z^{-1} J$, é central 
simples. Entretanto, como $\mathcal{M}$ é variedade homogênea (pois $F$ é infinito), $Z^{-1} J$ satisfaz as mesmas identidades de $J$, logo $Z^{-1} J$ é uma PI-álgebra de Jordan simples. Ainda pelo Teorema 3.2.7, $Z^{-1} J$ ou é de dimensão finita ou é do Tipo Clifford. Seja $B=Z^{-1} J$.

Suponha $B$ de tipo Albert. Neste caso,

$$
\bar{F} \otimes_{F} B \cong \mathcal{H}\left(M_{3}(\mathbb{O}), *\right)
$$

Entretanto, $\bar{F} \otimes_{F} B$ satisfaz as mesmas identidades de $B(\mathcal{M}$ é homogênea $), \log$ H $\mathcal{H}\left(M_{3}(\mathbb{O}), *\right)$ está em $\mathcal{M}$. Mas,

$$
\left\{\left[\begin{array}{lll}
\alpha & \delta & 0 \\
\delta & \beta & 0 \\
0 & 0 & 0
\end{array}\right], \alpha, \beta, \delta \in F\right\}
$$

é uma subálgebra de $\mathcal{H}\left(M_{3}(\mathbb{O}), *\right)$ isomorfa a $\mathcal{H}_{2}$.

Por hipótese, $\mathcal{H}_{2} \notin \mathcal{M}$, pelo que concluímos que uma álgebra do Tipo Albert não pode estar em $\mathcal{M}$.

Suponha que $B$ é do Tipo Clifford, isto é, $B=J(V, f)=V \dot{+} F$, em que $V$ é um espaço vetorial com uma forma bilinear não degenerada $f$. Se $\operatorname{dim}(V)<2$, então $B$ não será simples. Portanto precisamos analisar o caso em que $\operatorname{dim}(V) \geq 2$ (mais adiante, neste caso, encontraremos em $B$ uma subálgebra isomorfa a $\mathcal{H}_{2}$ ). Inicialmente, porque $\operatorname{dim}(V) \geq 2$, existe, ao menos, dois vetores linearmente independentes em $V$, digamos $v_{1}$ e $v_{2}$, pelos quais nós verificamos a não degeneração de $f$. Já que a mudança de base é ainda um automorfismo, podemos supor, sem perda de generalidade, que $f: V \times V \rightarrow F$ pode ser definida como a seguir:

$$
\begin{aligned}
& f\left(v_{1}, v_{1}\right)=1 \\
& f\left(v_{2}, v_{2}\right)=1 \\
& f\left(v_{1}, v_{2}\right)=0
\end{aligned}
$$

Agora vamos provar que a subálgebra $\mathcal{G}=\operatorname{Vect}_{F}\left\langle 1, v_{1}, v_{2}\right\rangle$ é isomorfa a $\mathcal{H}_{2}(F)$.

Os seguintes elementos formam uma base para $\mathcal{H}_{2}$ :

$$
a_{1}=\left[\begin{array}{ll}
1 & 0 \\
0 & 1
\end{array}\right], a_{2}=\left[\begin{array}{cc}
1 & 0 \\
0 & -1
\end{array}\right], a_{3}=\left[\begin{array}{ll}
0 & 1 \\
1 & 0
\end{array}\right]
$$

Considere o espaço vetorial $E=\operatorname{Vect}_{F}\left\langle a_{2}, a_{3}\right\rangle$. Primeiramente, note que $V e c t_{F}\left\langle a_{1}, a_{2}, a_{3}\right\rangle=$ $\mathcal{H}_{2}$ e $V e c t_{F}\left\langle a_{1}\right\rangle \cong F$ (como álgebra), isto é,

$$
\mathcal{H}_{2}(F)=\left\{\alpha a_{1}+\beta a_{2}+\gamma a_{3}, \alpha, \beta, \gamma \in F\right\}=F \dot{+} E .
$$

Considere a identificação natural entre "um escalar $\alpha \in F$ " e "uma matriz escalar (múltiplo escalar da matriz identidade)", isto é,

$$
\left[\begin{array}{ll}
\alpha & 0 \\
0 & \alpha
\end{array}\right] \cdot\left[\begin{array}{ll}
a & b \\
c & d
\end{array}\right]=\alpha\left[\begin{array}{ll}
a & b \\
c & d
\end{array}\right]
$$

Defina em $E$ uma forma bilinear $g$ como a própria multiplicação de Jordan para matrizes.

$$
g\left(\beta a_{2}+\gamma a_{3}, \mu a_{2}+\nu a_{3}\right)=\left[\begin{array}{cc}
\beta \mu+\gamma \nu & 0 \\
0 & \beta \mu+\gamma \nu
\end{array}\right] \leftrightarrow \beta \mu+\gamma \nu \in F .
$$


Claramente, esta $g$ é não degenerada. De acordo com esta descrição, $\mathcal{H}_{2}$ é uma álgebra do Tipo Clifford.

Esta construção mostra que o morfismo entre $\mathcal{G}$ e $\mathcal{H}_{2}$ dado por

$$
\begin{aligned}
1 & \mapsto a_{1} \\
v_{1} & \mapsto a_{2} \\
v_{2} & \mapsto a_{3}
\end{aligned}
$$

é um isomorfismo de $F$-álgebras.

Portanto, se $B$ é do Tipo Clifford, então $B$ conterá a cópia homomórfica de $\mathcal{H}_{2}$, contradizendo a hipótese.

Agora, suponha $B$ do Tipo Hermitiano.

Se $A$ é *-simples, então ou $A$ é simples com involução $*$ ou $A=\left(C \oplus C^{o p}, *\right)$, em que $C$ é simples e se $(x, y)$ é um elemento de $C \oplus C^{o p}$, então $(x, y)^{*}=(y, x)$.

Seja $\Omega$ o fecho algébrico de $F$. Neste caso, $\left(A_{\Omega}, *_{\Omega}\right)$ é também simples e a dimensão de $A_{\Omega}$ sobre $\Omega$ é a mesma de $A$ sobre $F$ (que é finita). Mais ainda, $B_{\Omega}=\mathcal{H}\left(A_{\Omega}, *_{\Omega}\right)$ é ainda um elemento da variedade $\mathcal{M}$ já que todas as identidades são mantidas $(\mathcal{M}$ é homogênea).

Há três possibilidades para $A_{\Omega}$ :

I : $\left(A_{\Omega}, *_{\Omega}\right)=\left(\Omega_{n}, t\right)$, em que $t: \Omega_{n} \rightarrow \Omega_{n}$ é a transposição de matrizes.

II $:\left(A_{\Omega}, *_{\Omega}\right)=\left(\Omega_{2 n}, *_{S}\right)$, em que $*_{S}: \Omega_{2 n} \rightarrow \Omega_{2 n}$ é involução simplética $X \mapsto S^{-1} X^{t} S$ e $S$ é a matriz diagonal de blocos $\operatorname{diag}\{Q, \cdots, Q\}$ ( $n$ vezes), $Q=\left[\begin{array}{cc}0 & 1 \\ -1 & 0\end{array}\right]$ e $X^{t}$ é a transposição de $X$ (imagem de $X$ pela involução "transposição").

III : $\left(A_{\Omega}, *_{\Omega}\right)=\left(\Omega_{n} \oplus \Omega_{n}, o\right)$, em que $\left(X, Y^{t}\right)^{o}=\left(Y, X^{t}\right)$.

Em todos os casos, $n$ é um natural.

Suponha que $A_{\Omega}$ é do tipo I. Se $n=1$, é claro que $A_{\Omega}=\Omega$ é um corpo e a involução $t$ é simplesmente o homomorfismo identidade sobre $\Omega$. Portanto,

$$
B_{\Omega}=\mathcal{H}\left(A_{\Omega}, *_{\Omega}\right)=\mathcal{H}(\Omega, \mathrm{id})=\Omega .
$$

Se $n \geq 2$, considere a subálgebra $\mathcal{H}_{2}$ de $\Omega_{n}$.

Note que $\mathcal{H}_{2}$ é uma subálgebra (de Jordan) de $\Omega_{n} t$-invariante, isto é, $t\left(\mathcal{H}_{2}\right) \subseteq \mathcal{H}_{2}$. Portanto, nós identificaremos a restrição da transposição $t$ sobre $F_{2}$ à própria transposição definida sobre $F_{2}$. este argumento será usado ao longo da demonstração e será chamado de argumento de identificação (da involução). Como é sabido $\mathcal{H}\left(\Omega_{n}, t\right)$ está em $\mathcal{M}$. Portanto, $\mathcal{H}_{2}=\mathcal{H}\left(F_{2}, t\right) \subseteq \mathcal{H}\left(\Omega_{n}, t\right)$ é também um elemento de $\mathcal{M}$, uma contradição.

Os próximos argumentos conclusivos serão dados de forma similar. Em $\mathcal{H}\left(\Omega_{n}, *_{S}\right)$ and $\mathcal{H}\left(\Omega_{n} \oplus \Omega_{n}, o\right)$ ou nós encontraremos uma cópia (homomórfica) de $\mathcal{H}_{2}(F)$ ou nós concluiremos que a álgebra de elementos hermitianos é meramente o corpo $\Omega$ (o caso unidimensional).

Suponha que $A_{\Omega}$ é do Tipo II. Primeiramente, identifiquemos os elementos hermitianos em $A_{\Omega}$. Seja $n=1$ (atentemo-nos à álgebra $\Omega_{2}$ ). Se $X \in \Omega_{2}$ é tal que $X^{*}=X$, então $X$ satisfaz a equação

$$
Q X=X^{t} Q, \text { em que } Q=\left[\begin{array}{cc}
0 & 1 \\
-1 & 0
\end{array}\right]
$$


Um cálculo simples nos mostra que $X$ deve ser um múltiplo da matriz identidade. Portanto, $\mathcal{H}\left(\Omega_{2}, *_{S}\right)=\Omega \cdot \mathrm{id}_{2}$, que é isomorfo ao próprio corpo $\Omega$. Logo, o caso $n=1$ é trivial aqui. Se $n=2$, considere a subálgebra $\left(F_{4}, *_{S}\right)$ de $\Omega_{4}$, em que $*_{S}$ é definido pelas identificação supracitada.

Considere em $\left(F_{4}, *_{S}\right)$ os seguintes elementos:

$$
v_{1}=\left[\begin{array}{cc}
\operatorname{id}\left(F_{2}\right) & 0 \\
0 & 0
\end{array}\right], v_{2}=\left[\begin{array}{cc}
0 & 0 \\
0 & \operatorname{id}\left(F_{2}\right)
\end{array}\right], v_{3}=\left[\begin{array}{cc}
0 & Q \\
-Q & 0
\end{array}\right] \text {. }
$$

Ademais, esses elementos são $*_{S}$-hermitianos, isto é, $*_{s}\left(v_{i}\right)=v_{i}$, para todo $i \in\{1,2,3\}$. A respeito de $v_{1}$ e $v_{2}$, este fato segue imediatamente. Para $v_{3}$, façamos o seguinte esquema:

$$
S^{-1} v_{3}^{t} S=\left[\begin{array}{cc}
-Q & 0 \\
0 & -Q
\end{array}\right]\left[\begin{array}{cc}
0 & Q \\
-Q & 0
\end{array}\right]\left[\begin{array}{cc}
Q & 0 \\
0 & Q
\end{array}\right]=\left[\begin{array}{cc}
0 & Q \\
-Q & 0
\end{array}\right]=v_{3} .
$$

Ao considerar a multiplicação de Jordan, note que

$$
v_{1}^{2}=v_{1} ; v_{2}^{2}=v_{2} ; v_{3}^{2}=v_{1}+v_{2} ; v_{1} v_{2}=0 ; 2 v_{1} v_{3}=2 v_{2} v_{3}=v_{3}
$$

Seja $\mathcal{G}=V e c t_{F}\left\langle v_{1}, v_{2}, v_{3}\right\rangle$, o subespaço vetorial de $F_{4}$ gerado por $v_{1}, v_{2}$ e $v_{3}$, e considere a álgebra de Jordan $(\mathcal{G},+, \cdot)$. Atentemo-nos que $*_{S}(\mathcal{G})=\mathcal{G}$. Agora, seja a álgebra de Jordan $\left(\mathcal{H}_{2}(F),+, \cdot\right)=\mathcal{H}\left(F_{2}, t\right)$, gerada (como espaço vetorial) pela base (de índices ordenados)

$$
\left\{\left[\begin{array}{ll}
1 & 0 \\
0 & 0
\end{array}\right],\left[\begin{array}{ll}
0 & 0 \\
0 & 1
\end{array}\right],\left[\begin{array}{ll}
0 & 1 \\
1 & 0
\end{array}\right]\right\}=\left\{w_{1}, w_{2}, w_{3}\right\} .
$$

A construção anterior mostra que a aplicação

$$
\begin{aligned}
& v_{1} \mapsto w_{1} \\
& v_{2} \mapsto w_{2} \\
& v_{3} \mapsto w_{3}
\end{aligned}
$$

é um isomorfismo de $F$-álgebras: uma contradição, pois $\mathcal{H}_{2} \notin \mathcal{M}$.

Também para o caso II, é claro que se $n>2$, então $\Omega_{2 n}$ conterá uma cópia de $\mathcal{H}_{4}$ e, dessa forma, conterá uma cópia de $\mathcal{H}_{2}$.

Vamos para o caso III. Se tomamos $n=1$, o caso será trivializado, já que a transposição $t$ é a própria involução identidade e a igualdade $(X, Y)=(Y, X)$ resulta em uma álgebra isomorfa ao corpo $\Omega$, como o caso do tipo II para $n=1$.

Seja $n=2$. Considere a subálgebra $F_{2} \oplus F_{2}$ de $\Omega_{2} \oplus \Omega_{2}$. nós procedemos de forma similar ao caso II. Defina para $\mathcal{H}_{2}$ a mesma base (de espaço vetorial) $\left\{w_{1}, w_{2}, w_{3}\right\}$. Os elementos $W_{1}=\left(w_{1}, w_{1}\right), W_{2}=\left(w_{2}, w_{2}\right)$ e $W_{3}=\left(w_{3}, w_{3}\right)$ são $o$-hermitianos, isto é, $W_{i}^{o}=W_{i}$, para todo $i \in\{1,2,3\}$ e $o: F_{2} \oplus F_{2} \rightarrow F_{2} \oplus F_{2}$ é a identificação da restrição da involução sobre $\Omega_{2} \oplus \Omega_{2}$.

Tais elementos satisfazem equações que resultarão em um isomorfismo de álgebras:

$$
W_{1}^{2}=W_{1} ; W_{2}^{2}=W_{2} ; W_{3}^{2}=W_{1}+W_{2} ; W_{1} W_{2}=0 ; 2 W_{1} W_{3}=2 W_{2} W_{3}=W_{3} .
$$

Portanto a subálgebra $V e c t\left\langle W_{1}, W_{2}, W_{3}\right\rangle$ of $\mathcal{H}\left(\Omega_{n} \oplus \Omega_{n}, o\right)$ é isomorfa (como álgebra) a 
$\mathcal{H}_{2}(F)$ através da aplicação

$$
\begin{aligned}
& W_{1} \mapsto w_{1} \\
& W_{2} \mapsto w_{2} \\
& W_{3} \mapsto w_{3} .
\end{aligned}
$$

Novamente, uma contradição com respeito a hipótese. É claro que se $n>2$, então $\Omega_{n} \oplus \Omega_{n}$ conterá uma cópia de $\Omega_{2} \oplus \Omega_{2}$ e, consequentemente, uma cópia de $\mathcal{H}_{2}(F)$.

em geral, concluímos que a única possibilidade do Tipo Hermitiano tem de ser $B_{\Omega}$ igual ao próprio corpo $\Omega$ e isso significa que $B=Z^{-1} J$ pode ser identificado como um subcorpo de $\Omega$, isto é, $B$ é uma álgebra associativa:

$$
(B, B, B)=(0)
$$

como $J$ satisfaz as mesmas identidades de $B$, concluímos que

$$
(J, J, J)=(0),
$$

isto é, $J$ é associativa.

De maneira similar aos trabalhos de Kemer, em [Kem80], nosso objetivo é descobrir quais identidades podem ser satisfeitas por variedades não matriciais de álgebras de Jordan.

A diferença está no modo de se obter os resultados. Seguindo Kemer, uma variedade não matricial satisfaz identidades. Nosso resultado vai no seguinte sentido: se a variedade satisfaz certas identidades, então a variedade é não matricial.

Segue-se o seguinte resultado (também original):

Proposição 3.3.2. Seja $\mathcal{V}$ uma variedade de F-álgebras de Jordan definida pela identidade

$$
(x, y, z)^{n} \equiv 0,
$$

para algum $n>0$ natural. Então $J$ é uma variedade não-matricial.

Demonstração. Aplicaremos a Proposição 3.3.1. Portanto, nos resta mostrar que $\mathcal{H}_{2} \notin \mathcal{V}$.

Considere a mesma base $\left\{a_{1}, a_{2}, a_{3}\right\}$ para $\mathcal{H}_{2}$ como na Proposição 3.3.1. Note que

$$
\left(a_{2}, a_{3}, a_{3}\right)=a_{2} \bullet\left(a_{3} \bullet a_{3}\right)=a_{2} .
$$

Logo, $\left(a_{2}, a_{3}, a_{3}\right)^{n}=a_{2}$ se $n$ é ímpar ou $\left(a_{2}, a_{3}, a_{3}\right)^{n}=1$ se $n$ é par. Em ambos os casos,

$$
\left(a_{2}, a_{3}, a_{3}\right)^{n} \neq 0, \quad \forall n \in \mathbb{N}
$$

então concluímos que $\mathcal{H}_{2}$ não está em $\mathcal{V}$. Logo, $\mathcal{V}$ é uma variedade não matricial.

Segue imediatamente de 3.3.1 e 3.3.2 o seguinte resultado:

Corolário 3.3.3. Seja J álgebra de Jordan prima não degenerada que satisfaz a identidade $(x, y, z)^{n}=0$, para algum natural $n$. Então $J$ é associativa. 
Nosso objetivo é apresentar uma caracterização para variedades não matriciais de álgebras de Jordan de forma similar ao Teorema 2, em [MPR2011]. Assim o fizemos, com ineditismo, no resultado a seguir.

Teorema A. Seja $\mathcal{M}$ uma variedade de álgebras de Jordan sobre um corpo infinito $F$ de caracteristica diferente de 2.

As seguintes condições são equivalentes:

(i) $\mathcal{H}_{2} \notin \mathcal{M}$, ou equivalentemente $\mathcal{M}$ é não matricial.

(ii) Seja $J \in \mathcal{M}$ álgebra finitamente gerada. Para algum s, J satisfaz a identidade

$$
\left(x_{1}, x_{2}, x_{3}\right) \cdot\left(x_{4}, x_{5}, x_{6}\right) \cdot \ldots \cdot\left(x_{3 s-2}, x_{3 s-1}, x_{3 s}\right) \equiv 0 .
$$

Os elementos $x_{i}$ são arbitrários e o produto da expressão acima não depende de uma combinação de parênteses, ainda que $J$ não seja associativa.

(iii) Existe $m$ tal que $\mathcal{M}$ satisfaz $(x, y, z)^{m} \equiv 0$.

(iv) Para todo $J \in \mathcal{M}$, os elementos nilpotentes de $J$ formam um ideal.

(v) Seja $J \in \mathcal{M}$. Qualquer conjunto finito $\left\{a_{1}, a_{2}, \cdots a_{k}\right\}$ de elementos algébricos sobre $J$ geram uma álgebra de dimensão finita $J_{0} \subseteq J$.

(vi) $S e J \in \mathcal{M}$ e $a \in J$ é nilpotente, então o ideal bilateral gerado por a é nil.

(vii) Se $J \in \mathcal{M}$ e $a, b \in J$ são elementos nilpotentes, então $a+b$ também é nilpotente.

Demonstração. Provemos tais equivalências.

(i) $\Longrightarrow$ (ii) Primeiramente, note que $\mathcal{M}$ é uma variedade homogênea ( $F$ é infinito). Seja $J$ uma álgebra de Jordan finitamente gerada e seja $\mathrm{Nil}(J)$ seu nil radical. Pelo Teorema 3.2.3, temos:

$$
J / \operatorname{Nil}(J) \hookrightarrow \prod_{J_{\alpha}} J_{\alpha},
$$

em que cada $J_{\alpha}$ é prima não degenerada com $\operatorname{Nil}\left(J_{\alpha}\right)=(0)$. Entretanto, $\operatorname{Degen}\left(J_{\alpha}\right) \subseteq$ $\operatorname{Nil}\left(J_{\alpha}\right)$, então cada $J_{\alpha}$ é uma PI-álgebra de Jordan prima não degenerada. Pela Proposição 3.3.1, cada $J_{\alpha}$ é associativa e isso significa que $J / \mathrm{Nil}(J)$ é associativa. Já que $J$ é finitamente gerada e PI, podemos afirmar (Medvedev, [Med88-2]) que seu nil radical, $\operatorname{Nil}(J)$, é nilpotente (Teorema 3.2.4). Seja $s$ o índice de nilpotência de $\operatorname{Nil}(J)$.

Como $\bar{J}$ é associativa, é sabido que $(\bar{J}, \bar{J}, \bar{J})=(\overline{0})$, isto é, $(J, J, J) \subseteq \operatorname{Nil}(J)$.

Logo $J$ satisfaz a identidade

$$
\left(x_{1}, x_{2}, x_{3}\right) \cdot\left(x_{4}, x_{5}, x_{6}\right) \cdot \ldots \cdot\left(x_{3 s-2}, x_{3 s-1}, x_{3 s}\right) \equiv 0
$$

que não depende de uma combinação de parênteses para a multiplicação, ainda que $J$ não seja associativa. 
(ii) $\Longrightarrow$ (iii) Seja $J_{\mathcal{M}}=F_{\mathcal{M}}[x, y, z]$ a $\mathcal{M}$-álgebra livre gerada por 3 elementos. Logo, ela satisfaz (3.2) para algum natural fixado $m=s$. Em particular, $(x, y, z)^{s}=0$ para os geradores $x, y, z \in J_{\mathcal{M}}$. Agora seja $J \in \mathcal{M}$ uma álgebra de Jordan arbitrária e $a, b, c \in J$. Pela propriedade universal das álgebras livres, a aplicação $x \mapsto a, y \mapsto b, z \mapsto c$, define um único homomorfismo $\varphi: J_{\mathcal{M}} \rightarrow J$. Temos

$$
0=\varphi(0)=\varphi\left((x, y, z)^{s}\right)=(\varphi(x), \varphi(y), \varphi(z))^{s}=(a, b, c)^{s} .
$$

Portanto a identidade $(x, y, z)^{m}$ é satisfeita para todas as álgebras em $\mathcal{M}$.

(iii) $\Longrightarrow$ (iv) Seja $J$ uma álgebra de Jordan que satisfaz $(x, y, z)^{n} \equiv 0$, para quaisquer $x, y$ e $z$ em $J$. Considere a álgebra quociente $\bar{J}=J / \operatorname{Nil}(J)$. Então $J$ é uma PI-álgebra de Jordan e $\operatorname{Nil}(\bar{J})=(\overline{0})$. Como

$$
\operatorname{Nil}(\bar{J}) \supseteq \operatorname{Degen}(\bar{J})
$$

temos $\operatorname{Degen}(\bar{J})=(\overline{0})$, isto é, $\bar{J}$ é não degenerada. Segue-se que

$$
\pi: \bar{J} \hookrightarrow \prod_{\alpha} J_{\alpha}
$$

em que cada $J_{\alpha}$ é prima, não degenerada e também satisfaz a identidade

$$
(x, y, z)^{n} \equiv 0 .
$$

Pelo Corolário 3.3.3, cada $J_{\alpha}$ é uma álgebra de Jordan associativa. Suponha que exista um elemento nilpotente $x$ que não pertence a $\mathrm{Nil}(J)$, isto é existe $m=m(x)$ tal que $\bar{x}^{m}=\overline{0}$, em que $\bar{x} \in \bar{J}$. Logo, $x^{m} \in \operatorname{Nil}(J)$, para algum $m$. Suponha, sem perda de generalidade, que $\bar{x}^{2}=0$. Temos que $\bar{J}$ é uma álgebra de Jordan associativa, $\bar{x} \bar{J}$ é um ideal de $\bar{J}$ e $(\bar{x} \bar{J})^{2}=(\overline{0})$. Entretanto, $\bar{x} \bar{J}$ é um ideal não nulo de $\bar{J}$. Finalmente, $\bar{x} \neq \overline{0}$ implica $\pi(\bar{x}) \neq 0$ em $\prod_{\alpha} J_{\alpha}$.

De acordo com esta construção, $\bar{x} \bar{J} \unlhd \bar{J}$ é um nil ideal não nulo, uma contradição, já que $(\overline{0})$ o único nil ideal de $\bar{J}=J / \operatorname{Nil}(J)$. Logo conclui-se que $x$ está em $\operatorname{Nil}(J)$, ou seja, o conjunto dos elementos nilpotentes forma um ideal.

(iv) $\Longrightarrow$ (i) Lembremos que $\mathcal{M}$ é uma variedade homogênea.

Logo, $\mathcal{H}_{2}(F) \in \mathcal{M}$ implica $\mathcal{H}_{2}(\bar{F}) \in \mathcal{M}$, em que $\bar{F}$ é o fecho algébrico de $F$.

Considere o elemento $i=\sqrt{-1}$ of $\bar{F}$. Veja que $X_{1}=\left[\begin{array}{cc}i & 1 \\ 1 & -i\end{array}\right]$ e $X_{2}=\left[\begin{array}{cc}-i & 1 \\ 1 & i\end{array}\right]$ são matrizes nilpotentes em $\mathcal{H}_{2}(\bar{F})$, mas $\frac{1}{2}\left(X_{1}+X_{2}\right)=\left[\begin{array}{ll}0 & 2 \\ 2 & 0\end{array}\right]$ não é nilpotente.

(i) $\Longrightarrow(\mathrm{v})$ Neste caso, é suficiente provar que, se uma álgebra $B$ é finitamente gerada por elementos algébricos, então é de dimensão finita.

Seja $B$ uma álgebra de Jordan finitamente gerada em $\mathcal{M}$. Seja $\mathcal{L}(B)=\mathcal{L}_{F}(B)$ seu radical localmente finito sobre $Z$ (veja a subseção 2.3.1, Teorema 2.3.1.24, [ZSh73]). Logo, $\bar{B}=B / \mathcal{L}(B)$ não tem $\mathcal{L}$-ideais não nulos e é isomorfo a uma soma subdireta de álgebras primas sem $\mathcal{L}$-ideais, digamos $\bar{B} \hookrightarrow \prod_{\alpha} B_{\alpha}$. Como $\operatorname{Deg}(B) \subseteq \operatorname{Locnil}(B)-$ observe que Locnil $=\mathcal{L}_{Z}$ para $Z=0-$, segue-se que cada $B_{\alpha}$ é também não degenerada. Pela Proposição 3.3.1, cada $B_{\alpha}$ é associativa. Logo $\bar{B}$ pode ser representado numa soma subdireta de 
álgebras primas associativas e commutativas. Neste caso, $\bar{B}$ é uma álgebra comutativa e associativa finitamente gerada por elementos algébricos, logo é uma $\mathcal{L}$-álgebra. Portanto $\mathcal{L}(\bar{B})=\bar{B}$. Por outro lado, $\mathcal{L}(\bar{B})=\mathcal{L}(B / \mathcal{L}(B))=(\overline{0})$, isto é, $\bar{B}=(\overline{0})$. Logo $B=\mathcal{L}(B)$, isto é, $B$ é localmente finita sobre $F$. Entretanto, $F$ é um corpo, logo $B$ é de dimensão finita.

$(\mathrm{v}) \Longrightarrow(\mathrm{i})$ Considere as mesmas matrizes de $(i v) \Longrightarrow(i)$. Suponha que qualquer álgebra em $\mathcal{M}$ finitamente gerada por elementos algébricos é de dimensão finita, mas $\mathcal{H}_{2} \in \mathcal{M}$; isto implica que $\mathcal{H}_{2}[x]$, a álgebra de polinômios em uma variável sobre $\mathcal{H}_{2}$, está em $\mathcal{M}$ (pois $\mathcal{M}$ é homogênea). $C_{1}=\left[\begin{array}{cc}i x & x \\ x & -i x\end{array}\right]$ e $C_{2}=\left[\begin{array}{cc}-i x & x \\ x & i x\end{array}\right]$. Note que $C_{1}^{2}=0=C_{2}^{2}$, isto é, $\operatorname{alg}\left\langle C_{1}, C_{2}\right\rangle$ é gerada (como álgebra) por elementos algébricos. Logo, $\left\langle C_{1}, C_{2}\right\rangle$ é de dimensão finita e algébrico, por hipótese. Em particular,

$C=\frac{1}{2}\left(C_{1}+C_{2}\right)=\left[\begin{array}{ll}0 & x \\ x & 0\end{array}\right]$ é algébrico. Isso significa que $C$ é algébrico se, e somente se, $x$ também o é, uma contradição, pois $C[x]$ não é de dimensão finita. Note que $C^{2 k+1}=$ $\left[\begin{array}{cc}0 & x^{2 k+1} \\ x^{2 k+1} & 0\end{array}\right]$ and $C^{2 k}=\left[\begin{array}{cc}x^{2 k} & 0 \\ 0 & x^{2 k}\end{array}\right]$. Portanto, $\mathcal{H}_{2} \notin \mathcal{M}$.

(i) $\Longrightarrow$ (vii) Os elementos nilpotentes formam um ideal, por (i) $\Longrightarrow$ (iv). Logo a soma de dois elementos nilpotentes é também um elemento do nil radical, portanto também nilpotente.

(vii) $\Longrightarrow(\mathrm{i}) X_{1}=\left[\begin{array}{cc}i & 1 \\ 1 & -i\end{array}\right]$ e $X_{2}=\left[\begin{array}{cc}-i & 1 \\ 1 & i\end{array}\right]$ são matrizes nilpotentes em $\mathcal{H}_{2}(\bar{F})$, mas $\frac{1}{2}\left(X_{1}+X_{2}\right)=\left[\begin{array}{ll}0 & 2 \\ 2 & 0\end{array}\right]$ não é nilpotente.

(i) $\Longrightarrow$ (vi) Provamos que (i) $\Longrightarrow$ (ii) $\Longrightarrow$ (iii) $\Longrightarrow$ (iv). Seja $I_{a}$ o ideal bilateral gerado por um elemento nilpotente $a$. Se $a \in \operatorname{Nil}(J)$, então, por (iv), $I_{a} \subseteq \operatorname{Nil}(J)$, isto é, $I_{a}$ é um nil ideal..

$($ vi $) \Longrightarrow\left(\right.$ i) Note que $X_{1}=\left[\begin{array}{cc}i & 1 \\ 1 & -i\end{array}\right]$ é nil e está em $\mathcal{H}_{2}(\bar{F})$, mas $\left(X_{1}\right)=M_{2}(\bar{F})$ não é nil.

Em particular, qualquer álgebra de Jordan $J$ numa variedade não matricial satisfaz as propriedades "se $a, b \in J$ são nilpotentes então $a+b$ é nilpotente" e "finitamente gerada (como álgebra) por elementos algébricos implica em dimensão finita (como espaço vetorial)".

Para outras classes de álgebras, é natural tentar-se definir uma noção de "não-matricial". Uma dificuldade que pode surgir é encontrar um bom "candidato matricial", isto é, uma álgebra matricial minimal de forma a definir a condição de "ser não matricial" para alguma outra variedade. No caso associativo, a álgebra minimal é $F_{2}$. Para álgebras de Jordan, esta álgebra minimal é $\mathcal{H}_{2}$. Para qualquer classe de álgebras, existe um candidato "universal" a ser escolhido para estabelecer a definição de "variedade não matricial"?

Vejamos as afirmações da Proposição 3.3.1. Ela nos mostra que álgebras de Jordan primas não degeneradas em uma variedade não matricial são associativas. Entretanto, estas condições são vistas de forma similar no trabalho de Mishchenko-Petrogradsky-Regev 
([MPR2011]) e Cekanu ([Cek79]). Eles utilizaram as propriedades das álgebras primitivas para caracterizar variedades não matriciais, pois álgebras primitivas em variedades não matriciais (de álgebras associativas) são corpos. Neste trabalho, estudamos álgebras de Jordan primas e não degeneradas no mesmo sentido: elas são associativas em variedades não matriciais (de Jordan). Nos casos associativo e de Jordan, álgebras simples em variedades não matriciais são corpos.

Tais observações nos motivam à seguinte definição:

Definição 5. Uma variedade $\mathcal{V}$ é dita ser não matricial se qualquer álgebra simples lá contida é um corpo.

Note que toda álgebra de Jordan simples é também prima não degenerada. Se $\mathcal{H}_{2} \notin \mathcal{V}$, logo a Definição 5 segue imediatamente da Proposição 3.3.1.

Por outro lado, se a Definição 5 é adotada, então $\mathcal{H}_{2} \notin \mathcal{V}$, já que $\mathcal{H}_{2}$ é uma álgebra de Jordan simples, mas não é um corpo.

No caso associativo, uma consequência similar a do Corolário 3.3.4 é também verdadeira (relembremos: uma variedade $\mathcal{M}$ de álgebras associativas é não matricial se $F_{2}$, a álgebra de matrizes $2 \times 2$ munida da multiplicação usual, não pertence a $\mathcal{M}$ ).

Dessa forma, temos a seguinte relação:

Corolário 3.3.4. As definições 4 e 5 são equivalentes para variedades de álgebras de Jordan.

O Corolário 3.3.4 sugere uma nova maneira de definir variedades não matriciais. Em vez de retirar um "tipo matricial minimal" de variedade de álgebras $\mathcal{V}$ (a fim de que $\mathcal{V}$ seja não matricial), estabeleceremos condições acerca das álgebras simples em $\mathcal{V}$. 


\section{Capítulo 4}

\section{Álgebras alternativas}

Agora, nosso objetivo é conseguir caracterizações não matriciais similares para álgebras alternativas.

Recordemos a definição de álgebras alternativas.

Definição 6 (Álgebra alternativa). Uma álgebra A será dita uma álgebra alternativa se ela satisfizer as seguintes identidades:

$$
(x, y, y)=0
$$

e

$$
(x, x, y)=0 \quad(\text { LAlt })
$$

A identidade RAlt, $(x, y, y)=0$, afirma que $\mathbf{A}$ é uma álgebra alternativa à direita. Por sua vez, a identidade LAlt, $(x, x, y)=0$ nos diz que A é uma álgebra alternativa à esquerda. Portanto, álgebras alternativas são alternativas à direita e à esquerda.

Segue, imediatamente da Definição 6, que álgebras associativas são alternativas também.

Mantendo a notação dos outros capítulos, $F_{2}$ é álgebra de matrizes quadradas $2 \times$ 2 sobre $F$ e $F$ é um corpo (na maioria dos casos, infinito). A variedade das álgebras alternativas sobre um anel $\Phi$ será denotada por Alt.

\subsection{Definições e exemplos}

Uma construção bastante conhecida na Matemática é processo de duplicação de CayleyDickson (para maiores detalhes, consulte [ZSSS82], Seção 2.2). Um exemplo desse processo é a construção da álgebra dos octônios usuais:

$$
\mathbb{R} \rightarrow \mathbb{R} \overline{\times} \mathbb{R} \cong \mathbb{C} \rightarrow \mathbb{C} \overline{\times} \mathbb{C} \cong \mathbb{H} \rightarrow \mathbb{H} \overline{\times} \mathbb{H} \cong \mathbb{O} .
$$

As álgebras $\mathbb{R}$ (o corpo dos reais), $\mathbb{C}$ (o corpo dos complexos), $\mathbb{H}$ (a álgebra dos quatérnios) e $\mathbb{O}$ (a álgebra dos octônios) são álgebras de divisão, isto é, qualquer elemento não nulo nestas álgebras admite inverso multiplicativo. O processo de duplicação especificado como acima pontua algumas "perdas" em cada dos seus passos

- $\mathbb{R}$ é álgebra de divisão, comutativa, associativa, munida de uma ordem " $\leq$ " compatível com suas operações usuais de adição e multiplicação; 
- $\mathbb{C}$ é álgebra de divisão, comutativa, associativa;

- $\mathbb{H}$ é álgebra de divisão, associativa;

- (1) é álgebra de divisão.

De fato, $\mathbb{H}$ é associativa, mas não é comutativa e $\mathbb{O}$ não é associativa nem comutativa.

A álgebra $\mathbb{O}$ pode ser assim definida: considere $\mathbb{R}$-espaço vetorial de oito dimensões $\mathbb{R}^{8}$. Seja $\left\{1, e_{1}, \cdots, e_{7}\right\}$ uma base para $\mathbb{R}^{8}$ e defina uma multiplicação em $\mathbb{R}^{8}$ a partir dos elementos da base, através da seguinte tabela:

\begin{tabular}{|c|ccccccc|}
\hline$\cdot$ & $e_{1}$ & $e_{2}$ & $e_{3}$ & $e_{4}$ & $e_{5}$ & $e_{6}$ & $e_{7}$ \\
\hline$e_{1}$ & -1 & $e_{3}$ & $-e_{2}$ & $e_{5}$ & $-e_{4}$ & $-e_{7}$ & $e_{6}$ \\
$e_{2}$ & $-e_{3}$ & -1 & $e_{1}$ & $e_{6}$ & $e_{7}$ & $-e_{4}$ & $-e_{5}$ \\
$e_{3}$ & $e_{2}$ & $-e_{1}$ & -1 & $e_{7}$ & $-e_{6}$ & $e_{5}$ & $-e_{4}$ \\
$e_{4}$ & $-e_{5}$ & $-e_{6}$ & $-e_{7}$ & -1 & $e_{1}$ & $e_{2}$ & $e_{3}$ \\
$e_{5}$ & $e_{4}$ & $-e_{7}$ & $e_{6}$ & $-e_{1}$ & -1 & $-e_{3}$ & $e_{2}$ \\
$e_{6}$ & $e_{7}$ & $e_{4}$ & $-e_{5}$ & $-e_{2}$ & $e_{3}$ & -1 & $-e_{1}$ \\
$e_{7}$ & $-e_{6}$ & $e_{5}$ & $e_{4}$ & $-e_{3}$ & $-e_{2}$ & $e_{1}$ & -1 \\
\hline
\end{tabular}

Se $x=\left(x_{0}, \cdots, x_{7}\right), y=\left(y_{0}, \cdots, y_{7}\right) \in \mathbb{R}^{8}$ são elementos arbitrários, então

$$
\begin{gathered}
x=x_{0}+x_{1} e_{1}+x_{2} e_{2}+\cdots+x_{7} e_{7}, \\
y=y_{0}+y_{1} e_{1}+y_{2} e_{2}+\cdots+y_{7} e_{7},
\end{gathered}
$$

são multiplicados a partir de cada elemento da base através da tabela acima. A estrutura $\mathbb{O}=\left(\mathbb{R}^{8},+, \cdot\right)$ é a álgebra de octônios usual, também conhecida como álgebra de Cayley. A álgebra de Cayley é uma álgebra alternativa.

\subsection{Radicais em Alt}

De forma análoga às álgebras de Jordan, é possível determinar em Alt alguns radicais bastante conhecidos na literatura.

Teorema 4.2.1. A classe de todas as álgebras localmente nilpotentes é radical dentro da variedade das álgebras alternativas.

Teorema 4.2.2. Seja Locnil o radical localmente nilpotente de uma certa variedade de álgebras $\mathcal{V}$ para a qual o radical Locnil esteja bem definido. Para toda álgebra $A \in \mathcal{V}$, o radical Locnil $(A)$ é igual a intersecção de todos os ideais primos $P_{\alpha}$ de $A$ tais que a álgebra quociente $A / P_{\alpha}$ é Locnil-semissimples.

Em Alt ainda é possível definir o radical primo (ou radical de Baer), todavia este radical não será útil a este trabalho e deixamos a referência [ZSSS82] ao leitor interessado acerca da construção deste radical.

Na subseção 2.3.1, estabelecemos os seguintes radicais para uma álgebras alternativa A arbitrária:

Os resultados exibidos nesta subseção, particularmente se A é uma álgebra em Jord $\cup$ Alt $\cup[-1,1]$, nos permitem determinar a existência dos seguintes radicais: 
- $\mathrm{Nil}(\mathbf{A})$, o nil radical de A, também conhecido como nil radical superior ou radical de Köethe.

- Locnil(A), o radical localmente nilpotente de A, também conhecido como radical de Levitzki.

- $\mathfrak{a l g}(\mathbf{A})$, o radical algébrico de $\mathbf{A}$.

- $\mathcal{L}_{Z}(\mathrm{~A})$, o radical localmente finito (sobre um certo ideal $Z$ do anel de operadores de $\mathbf{A}$ ), que induz a existência de um radical de dimensão finita local, se a álgebra alternativa é sobre um corpo.

Considere o seguinte teorema de estrutura, ainda da mesma subseção

Teorema 4.2.3 (Shestakov [ZSh73]). Se A é uma álgebra alternativa, então $\mathbf{A} / \mathcal{L}_{F}(\mathbf{A})$ é isomorfa a uma soma subdireta de álgebras alternativas primas $\mathcal{L}_{F}$-semissimples:

$$
\mathbf{A} / \mathcal{L}_{F}(\mathbf{A}) \hookrightarrow \prod \mathbf{A}_{\alpha}, \quad \mathbf{A}_{\alpha} \text { é prima e } \mathcal{L}_{F}\left(\mathbf{A}_{\alpha}\right)=(0), \forall \alpha .
$$

Agora, nosso desenvolvimento consistirá em definir o análogo do radical de Jacobson para álgebras alternativas.

Seja A uma álgebra alternativa. Um ideal à direita $I$ de $\mathbf{A}$ é dito ser modular se existe um elemento $e \in \mathbf{A}$ tal que $e a-a \in I$, para todo $a \in \mathbf{A}$. Analogamente, um ideal à esquerda $I$ é dito ser modular se existe um elemento $e \in \mathbf{A}$ tal que $a e-a \in I$, para todo $a \in \mathbf{A}$.

Proposição 4.2.4. Seja A uma álgebra alternativa e seja I um ideal à direita modular de A. Então, o maior ideal $\tilde{I}$ da álgebra $\mathbf{A}$ contido em I coincide com o conjunto (I : $A)=\{a \in \mathbf{A} ; A a \subset I\}$.

Definição 4.2.5 (Álgebra Primitiva). Diremos que uma álgebra alternativa A é primitiva (à direita) se ela possui um ideal à direita modular maximal $I$ que não contém ideais bilaterais de A. Analogamente, diremos que uma álgebra alternativa A é primitiva (à esquerda) se ela possui um ideal à esquerda modular maximal $J$ que não contém ideais bilaterais de $\mathbf{A}$. Por fim, uma álgebra $\mathbf{A}$ é dita ser primitiva se o é à esquerda e à direita.

Pela Proposição 4.2.4, a relação $\tilde{I}=(I: \mathbf{A})=(0)$ é válida para o ideal $I$. O análogo vale na definição à esquerda.

De forma usual, um ideal bilateral $B$ de $\mathbf{A}$ é dito ser primitivo se a álgebra quociente A/ $B$ é uma álgebra alternativa primitiva.

O seguinte resultado nos será útil:

Teorema 4.2.6 (Kleinfeld). Toda álgebra alternativa primitiva ou é uma álgebra associativa primitiva ou uma álgebra de Cayley-Dickson sobre seu centro.

Kleinfeld demonstra que os seguintes conjuntos coincidem:

$$
\begin{aligned}
& K_{1}=\cap\{I \subseteq \mathbf{A} ; I \text { é ideal à direita maximal modular }\} \\
& K_{2}=\cap\{J \subseteq \mathbf{A} ; J \text { é ideal à esquerda maximal modular }\} \\
& K_{3}=\cap\{B \subseteq \mathbf{A} ; B \text { é ideal bilateral primitivo }\} .
\end{aligned}
$$


Em outras palavras, se A é uma álgebra alternativa, então a intersecção de todos os ideais à direita maximais modulares, a intersecção de todos os ideais à esquerda maximais modulares e a intersecção de todos os ideais bilaterais primitivos coincidem!

Denotemos esse conjunto por $\operatorname{Jac}(\mathbf{A})$. Ademais, o próprio Kleinfeld demonstra que $\operatorname{Jac}(\mathbf{A})$ é um ideal bilateral em $\mathbf{A}$.

Considere em Alt a seguinte classe de álgebras: Jac $=\{\mathbf{A} \in \mathrm{Alt} \mid \mathbf{A}=\operatorname{Jac}(\mathbf{A})\}$.

Teorema 4.2.7. A classe Jac é radical em Alt.

Posteriormente, Zhevlakov estabelece uma relação radical entre o ideal $\operatorname{Jac}(\mathbf{A})$ e elementos quase-regulares ${ }^{1}$ de $\mathbf{A}$. Desta forma, define-se o análogo do radical de Jacobson para álgebras alternativas, conhecido radical de Zhevlakov ou radical quase-regular.

Teorema 4.2.8 (Zhevlakov, [ZSSS82]). Se A é uma álgebra alternativa, então A/Jac(A) é isomorfa a uma soma subdireta de álgebras alternativas primitivas.

$$
\mathbf{A} / \operatorname{Jac}(\mathbf{A}) \hookrightarrow \prod \mathbf{A}_{\alpha}, \quad \mathbf{A}_{\alpha} \text { é primtiva } \forall \alpha
$$

Aqui não é necessário escrever "álgebras alternativas primitivas Jac-semissimples", pois álgebras alternativas primitivas são sempre Jac-semissimples.

Em [Sh83], o seguinte resultado é estabelecido:

Teorema 4.2.9. Se A é uma PI-álgebra alternativa finitamente gerada, então Jac(A) é nilpotente.

Fixemo-nos nos seguintes radicais definidos na classe das álgebras alternativas

$\diamond \mathcal{L}(\mathbf{A})$ é o radical localmente finito da álgebra alternativa $\mathbf{A}$.

$\diamond \operatorname{Jac}(\mathbf{A})$ é o radical de Zhevlakov (um análogo do radical de Jacobson para álgebras alternativas) da álgebra alternativa $\mathbf{A}$, também conhecido como radical quase-regular.

\subsection{Variedades não matriciais em Alt}

Consideremos a Definição 5 apresentada no capítulo anterior para álgebra alternativas, isto é,

Definição 4.3.1. Diremos que uma variedade $\mathcal{M}$ de álgebras alternativas é não matricial se toda álgebra simples contida lá é um corpo.

Consideremos, portanto, a seguinte Proposição.

Proposição 4.3.2. Seja $\mathcal{V}$ uma variedade de álgebras alternativas sobre um corpo infinito $F$ de característica diferente de 2. Se $F_{2} \notin \mathcal{M}$ e $\mathbf{A} \in \mathcal{M}$ é uma álgebra alternativa primitiva, então A é um corpo.

\footnotetext{
${ }^{1}$ Um elemento $a$ da álgebra alternativa $\mathbf{A}$ é dito ser quase-regular se existe um elemento $b$ tal que $a b=b a=a+b$. Uma álgebra alternativa $\mathbf{A}$ é dita ser quase-regular se todos os seus elementos são quase-regulares. A classe das álgebras quase-regulares é radical em Alt e Zhevlakov demonstra que esta classe coincide com a classe Jac definida nesta seção.
} 
Demonstração. Usaremos o Teorema 4.2.6. Suponha que A seja não associativa. Então, $Z^{-1} A$ é isomorfo a álgebra de octônios, em que $Z$ é o centro de A. Mostraremos que isso contradiz a hipótese $F_{2} \notin \mathcal{M}$.

A variedade $\mathcal{M}$ é homogênea, já que $F$ é inifinito, então $Z^{-1} A \in \mathcal{M}$. O processo de Cayley-Dickson (para mais detalhes, consulte [ZSSS82], seção 2.2) para $Z^{-1} A$ nos fornece $Z^{-1} A=(\mathbb{H}, \alpha)$, em que $\mathbb{H}$ é a álgebra dos quatérnios generalizada e $\alpha$ é o centro de $\mathbf{A}$. Logo $\mathbb{H} \in \mathcal{M}$ também. Seja $\bar{F}$ o fecho algébrico de $F$. Note que

$$
\bar{F} \otimes_{F} \mathbb{H I} \cong \bar{F}_{2} \text {. }
$$

Portanto $\bar{F}_{2} \in \mathcal{M}$. Entretanto, $F_{2}$ é uma subálgebra de $\bar{F}_{2}$, logo $F_{2} \in \mathcal{M}$, uma contradição.

Logo concluímos que A associativa, portanto (Teorema 4.2.6) é primitiva.

Repetindo as construções em [MPR2011] (Teorema 1), uma álgebra associativa primitiva que pertence a uma variedade não matricial (nesta demonstração, o conceito coincide com o caso associativo, pois $\left.F_{2} \notin \mathcal{V}\right)$ é um corpo.

Corolário 4.3.3. De acordo com a Definição 4.3.1, uma variedade $\mathcal{M}$ de álgebras alternativas é não matricial se, e somente se, $F_{2} \notin \mathcal{M}$.

Demonstração. Uma PI-álgebra alternativa simples é primitiva também (veja [ZSSS82]). O resultado segue imediatamente de 4.3.2.

Demonstremos o análogo do Teorema 2 para álgebras alternativas. Este resultado é também inédito e faz parte do trabalho de pesquisa desta tese.

Teorema B. Seja $\mathcal{M}$ uma variedade de álgebras alternativas sobre um corpo infinito $F$ de caracteristica diferente de 2.

As seguintes condições são equivalentes:

i) $\mathcal{M}$ é não matricial.

ii) Seja $\mathbf{A} \in \mathcal{M}$ uma álgebra finitamente gerada. Para algum s, A satisfaz a identidade

$$
u_{1} u_{2} \ldots u_{n} \equiv 0,
$$

em que cada $u_{i}$ ou é comutador ou é associador de elementos arbitrários em $\mathbf{A}$. $O$ produto da identidade 4.1 independe de uma combinação de parênteses, mesmo que A seja não associativa.

iii) Para qualquer $\mathbf{A} \in \mathcal{M}$, existe $m$ tal que A satisfaz $(x, y, z)^{m} \equiv 0$ e $[x, y]^{m} \equiv 0$, para quaisquer elementos $x, y, z \in \mathbf{A}$.

iv) Para qualquer $\mathbf{A} \in \mathcal{M}$, os elementos nilpotentes de $\mathbf{A}$ formam um ideal.

v) $S e \mathbf{A} \in \mathcal{M}$ e $a, b \in A$ são elementos nilpotentes, então $a+b$ também é nilpotente.

vi) Se $\mathbf{A} \in \mathcal{M}$ e $a \in A$ é nilpotente, então o ideal bilateral gerado por a é nil.

vii) Se $\mathbf{A} \in \mathcal{M}$ e $a \in A$ é nilpotente, então o ideal à esquerda $I_{l}$ (ou o ideal à direita $\left.I_{r}\right)$ gerado por a é nil. 
viii) Seja $\mathbf{A} \in \mathcal{M}$. Qualquer conjunto finito $\left\{a_{1}, a_{2}, \cdots a_{k}\right\}$ de elementos algébricos sobre A gera uma álgebra de dimensão finita $\mathbf{A}_{0} \subseteq A$.

Demonstração.

(i) $\Longrightarrow$ (ii) Seja A uma álgebra alternativa finitamente gerada e seja Jac(A) radical de Zhevlakov. O Teorema 4.2.8 nos assegura que

$$
\overline{\mathbf{A}}=A / \operatorname{Jac}(\mathbf{A}) \hookrightarrow \prod_{B_{\alpha}} \text { primitive } B_{\alpha}
$$

em que cada $B_{\alpha}$ é PI-álgebra alternativa primitiva, logo cada $B_{\alpha}$ é um corpo (Lema 4.3.2). Como A é finitamente gerada e PI, podemos afirmar que (Teorema 4.2.9) seu radical de Zhevlakov é nilpotente. Seja $s$ o índice de nilpotência de $\operatorname{Jac}(\mathbf{A})$.

Como $\overline{\mathbf{A}}$ é comutativa, $[\overline{\mathbf{A}}, \overline{\mathbf{A}}]=(\overline{0})$, ou seja, $[\mathbf{A}, \mathbf{A}] \subseteq \operatorname{Jac}(\mathbf{A})$. Como $\overline{\mathbf{A}}$ é associativa, $(\overline{\mathbf{A}}, \overline{\mathbf{A}}, \overline{\mathbf{A}})=(\overline{0})$, ou seja, $(\mathbf{A}, \mathbf{A}, \mathbf{A}) \subseteq \operatorname{Jac}(\mathbf{A})$. Como $\operatorname{Jac}(\mathbf{A})$ é nilpotente, A satisfaz a identidade

$$
u_{1} \cdots u_{s} \equiv 0,
$$

em que cada $u_{i}$ ou é associador ou é comutador. Esta identidade que não depende de uma combinação de parênteses, mesmo que A não seja associativa.

(ii) $\Longrightarrow$ (iii) Seja $\mathbf{A}_{\mathcal{M}}=F_{\mathcal{M}}[x, y, z]$ a álgebra $\mathcal{M}$-livre finitamente gerada por 3 elementos. Logo ela satisfaz 4.1 para algum natural $m=s$ fixado. Em particular, $[x, y]^{s}=0=$ $(x, y, z)^{s}$, para os geradores $x, y \in A_{\mathcal{M}}$. Agora, seja $\mathbf{A} \in \mathcal{M}$ uma álgebra de Jordan arbitrária e $a, b \in A$. Pela propriedade universal das álgebras livres, a aplicação $x \mapsto a$, $y \mapsto b$ define um único homomorfismo $\varphi: A_{\mathcal{M}} \rightarrow A$. Temos

$$
0=\varphi(0)=\varphi\left([x, y]^{s}\right)=[\varphi(x), \varphi(y)]^{s}=[a, b]^{s} .
$$

Portanto, a identidade $[x, y]^{m}$ é satisfeita por todas as álgebras em $\mathcal{M}$. De forma similar, a identidade $(x, y, z)^{m}$ é satisfeita por todas as álgebras em $\mathcal{M}$.

(iii) $\Longrightarrow$ (iv) Esta demonstração pode ser encontrada em [ZSSS82], Capítulo 7, Proposição 2 .

(iv) $\Longrightarrow$ (i) $E_{21}, E_{12} \in F_{2}$ são nil ${ }^{2}$, mas $E_{21}+E_{12}$ não é nil.

(i) $\Longrightarrow$ (v) Os elementos nilpotentes formam um ideal, por (i) $\Leftrightarrow$ (iv). Logo, a soma de elementos nilpotentes está no nil radical, ou seja, a soma de dois elementos nilpotentes é nilpotente. .

$(\mathrm{v}) \Longrightarrow(\mathrm{i})$ Note que $E_{11}+E_{12}$ pertence ao ideal à direita $E_{12} F_{2}$. Entretanto, $E_{11}+E_{12}$ é idempotente.

\footnotetext{
${ }^{2}$ Usamos a notação usual para matrizes. Uma matriz elementar do tipo $E_{k l}=\left(a_{i j}\right)$ é aquela em que $a_{k l}=1$ e $a_{i j}=0$, se $i \neq k$ ou $j \neq l$.
} 
(i) $\Longrightarrow$ (vi) Se $a \in A$ é nilpotente, então ( $a$ ) é uma subálgebra do ideal gerado por elementos nilpotentes. De (i) $\Leftrightarrow$ (iv), (a) está no nil ideal, portanto (a) é nil.

(vi) $\Longrightarrow$ (i) Suponha $F_{2} \in \mathcal{M}$. O elemento $E_{12}$ é nilpotente, mas, como $F_{2}$ é simples, $\left(E_{12}\right)=F_{2}$ não é nil.

(i) $\Longrightarrow$ (vii) Provamos (i) $\Leftrightarrow$ (vii). Claro, (vii) implica (viii), então o resultado segue.

(vii) $\Longrightarrow\left(\right.$ i) Note que $E_{12}$ é nilpotente, mas $\left(E_{12}\right)_{r}$ e $\left(E_{12}\right)_{l}$ não são nil.

(i) $\Longrightarrow$ (viii) A demonstração é similar ao caso de álgebras de Jordan (Teorema A).

Seja $B$ uma álgebra alternativa finitamente gerada por elementos algébricos em $\mathcal{M}$. Seja $\mathcal{L}(B)$ seu radical localmente finito ([ZSh73]).

Logo $\bar{B}=B / \mathcal{L}(B)$ não tem $\mathcal{L}$-ideais e é isomorfa a uma soma subdireta de álgebras primas sem $\mathcal{L}$-ideais não nulos (Teorema 4.2.3), digamos $\bar{B} \hookrightarrow \prod_{\alpha} B_{\alpha}$.

Portanto $\bar{B}$ é representada como uma soma subdireta de álgebras associativas e comutativas (Lema 4.3.2).

Neste caso, $\bar{B}$ também é uma álgebra comutativa e associativa finitamente gerada por elementos algébricos, logo é de dimensão finita (c. [Cek79]), portanto é localmente finita. Logo $\mathcal{L}(\bar{B})=\bar{B}$. Por outro lado, $\mathcal{L}(\bar{B})=\mathcal{L}(B / \mathcal{L}(B))=(\overline{0})$, isto é, $\bar{B}=(\overline{0})$. Concluímos que $B=\mathcal{L}(B)$, ou seja, $B$ é uma álgebra localmente finita sobre um corpo $F$, portanto é de dimensão finita.

(viii) $\Longrightarrow$ (i) Suponha que qualquer álgebra em $\mathcal{M}$, que é finitamente gerada por elementos algébricos, seja de dimensão finita, mas $F_{2} \in \mathcal{M}$. Isso implica que $F_{2}[x] \in \mathcal{M}(\mathcal{M}$ é homogênea e $F_{2}[x]$ é o anel de polinômios sobre $F_{2}$ ).

Defina $C_{1}=\left[\begin{array}{ll}0 & x \\ 0 & 0\end{array}\right]$ e $C_{2}=\left[\begin{array}{ll}0 & 0 \\ x & 0\end{array}\right]$. Note que $C_{1}^{2}=0=C_{2}^{2}$ e $\left\langle C_{1}, C_{2}\right\rangle$ é uma álgebra (finitamente) gerada por elementos algébricos. Logo $\left\langle C_{1}, C_{2}\right\rangle$ é de dimensão finita, por hipótese. Também, $C=\frac{1}{2}\left(C_{1}+C_{2}\right)=\left[\begin{array}{ll}0 & x \\ x & 0\end{array}\right]$ é algébrico. Isso significa que $C$ é algébrico se, e somente se, $x$ é algébrico, uma contradição, pois $C[x]$ não é de dimensão finita. Note que $C^{2 k+1}=\left[\begin{array}{cc}0 & x^{2 k+1} \\ x^{2 k+1} & 0\end{array}\right]$ and $C^{2 k}=\left[\begin{array}{cc}x^{2 k} & 0 \\ 0 & x^{2 k}\end{array}\right]$.

Portanto, $F_{2} \notin \mathcal{M}$.

Nosso próximo passo é estabelecer o análogo para uma classe de álgebras alternativas à direita.

\subsection{O caso não matricial para $(-1,1)$-álgebras}

Definição 7 ((-1,1)-álgebra). Uma álgebra A será dita uma (-1,1)-álgebra se ela satisfizer as seguintes identidades:

$$
(x, y, y)=0
$$


$\mathrm{e}$

$$
(x, y, z)+(y, z, x)+(z, x, y)=0
$$

Consideremos álgebras $(-1,1)$ como álgebras sobre um corpo $F$ de característica diferente de 2 ou de 3, que satisfazem as identidades acima descritas. Ou seja, uma $(-1,1)$ álgebra é uma álgebra alternativa à direita que satisfaz uma identidade cíclica para o associador. Denotemos a variedade as álgebras $(-1,1)$ por $[-1,1]$.

Esta classe de álgebras forma uma outra subclasse de álgebras alternativas à direita que contém as álgebras associativas

$$
[-1,1] \cap \mathrm{Alt}=\text { Assoc }
$$

Como no caso associativo, adotaremos a seguinte definição

Definição 8. Uma variedade $\mathcal{M}$ de $(-1,1)$-álgebras é dita ser não matricial se $F_{2} \notin \mathcal{M}$.

Conforme já mencionado na subseção 2.3.1, os seguintes radicais estão definidos em uma (-1,1)-álgebra A:

- $\mathrm{Nil}(\mathbf{A})$, o nil radical de $\mathbf{A}$, também conhecido como nil radical superior ou radical de Köethe.

- Locnil(A), o radical localmente nilpotente de A, também conhecido como radical de Levitzki.

- $\mathfrak{a l g}(\mathbf{A})$, o radical algébrico de $\mathbf{A}$.

- $\mathcal{L}_{Z}(\mathbf{A})$, o radical localmente finito (sobre um certo ideal $Z$ do anel de operadores de $\mathbf{A}$ ), que induz a existência de um radical de dimensão finita local.

Em [Hen72], encontramos o seguinte resultado:

Teorema 4.4.1 (Hentzel). Seja A uma (-1,1)-álgebra Nil-semissimples. Então A é associativa.

Ainda sobre o nil radical em álgebras $(-1,1)$, temos o seguinte resultado em [Pch04]:

Teorema 4.4.2 (Pchelintsev). Se A é uma PI-álgebra (-1,1) finitamente gerada, então $\mathrm{Nil}(\mathrm{A})$ é nilpotente.

Seja A uma álgebra $(-1,1)$. Seja $\mathcal{D}$ a subálgebra de $\mathbf{A}$ gerada por todos associadores de $\mathbf{A}$, isto é,

$$
\mathcal{D}=\mathcal{D}(\mathbf{A})=\operatorname{alg}\langle(x, y, z) \mid x, y, z \in \mathbf{A}\rangle .
$$

Pode-se demonstrar (consulte [Hen69]) que $\mathcal{D}$ é um ideal de A. Em virtude deste fato, $\mathcal{D}$ é chamado de ideal associador de A. Em [Pch75], demonstra-se o seguinte:

Teorema 4.4.3 (Pchelintsev). O ideal associador de qualquer álgebra $(-1,1)$ é localmente nilpotente. 
O próximo resultado nos será útil.

Lema 4.4.4. Seja $\mathcal{M}$ uma variedade não matricial de (-1,1)-álgebras. Se $A \in \mathcal{M}$ é uma álgebra Nil-semissimples, então A é associativa e comutativa.

Demonstração. Pelo Teorema 4.4.1, devido a Hentzel, $A / \operatorname{Nil}(\mathbf{A})$ é associativa.

Ademais, A está em $\mathcal{M}$ que é não matricial (pois $F_{2} \notin \mathcal{M}$ ), é associativa e Nilsemissimples, logo segue-se que $A$ é comutativa.

Estamos aptos a demonstrar a versão não matricial das álgebras $(-1,1)$. Queremos destacar também que tal resultado foi obtido originalmente nesta tese.

Teorema C. Seja $\mathcal{M}$ uma variedade de (-1,1)-álgebras sobre um corpo $F$ infinito de característica diferente de 2 ou de 3.

As seguintes condições são equivalentes:

i) $\mathcal{M}$ é não matricial.

ii) Seja $\mathbf{A} \in \mathcal{M}$ uma álgebra finitamente gerada. Para algum s, A satisfaz a identidade

$$
u_{1} u_{2} \ldots u_{n} \equiv 0
$$

em que cada $u_{i}$ ou é comutador ou é associador de elementos arbitrários em $\mathbf{A}$. $O$ produto da identidade 4.2 independe de uma combinação de parênteses, mesmo que A seja não associativa.

iii) Para qualquer $\mathbf{A} \in \mathcal{M}$, existe $m$ tal que A satisfaz $(x, y, z)^{m} \equiv 0$ e $[x, y]^{m} \equiv 0$, para quaisquer elementos $x, y, z \in \mathbf{A}$.

iv) Para qualquer $\mathbf{A} \in \mathcal{M}$, os elementos nilpotentes de $\mathbf{A}$ formam um ideal.

v) Seja $\mathbf{A} \in \mathcal{M}$. Qualquer conjunto finito $\left\{a_{1}, a_{2}, \cdots a_{k}\right\}$ de elementos algébricos sobre A gera uma álgebra de dimensão finita $A_{0} \subseteq A$.

vi) $S e \mathbf{A} \in \mathcal{M}$ e $a, b \in \mathbf{A}$ são elementos nilpotentes, então $a+b$ também é nilpotente.

vii) Se $\mathbf{A} \in \mathcal{M}$ e $a \in \mathbf{A}$ é nilpotente, então o ideal bilateral gerado por a é nil.

viii) Se $\mathbf{A} \in \mathcal{M}$ e $a \in \mathbf{A}$ é nilpotente, então o ideal à esquerda gerado por a (ou o ideal à direita gerado por a) é nil.

Demonstração. As técnicas aqui usadas diferirão um pouco das usadas no Teorema B.

(i) $\Longrightarrow$ (ii): Note que $\mathcal{M}$ é uma variedade homogênea ( $F$ é infinito). Seja $\mathbf{A}$ uma álgebra $(-1,1)$ finitamente gerada e seja $\mathrm{Nil}(\mathbf{A})$ o seu nil radical.

Pelo Lema 4.4.4, $\overline{\mathbf{A}}=\mathbf{A} / \operatorname{Nil}(\mathbf{A})$ é associativa e comutativa. Como $\mathbf{A}$ é finitamente gerada e $P I$, podemos afirmar que $\mathrm{Nil}(\mathbf{A})$ é nilpotente (Teorema 4.4.2), suponhamos de índice $s$.

Como $\overline{\mathbf{A}}$ é associativa, é sabido que $(\overline{\mathbf{A}}, \overline{\mathbf{A}}, \overline{\mathbf{A}})=(\overline{0})$, isto é $(\mathbf{A}, \mathbf{A}, \mathbf{A}) \subseteq \operatorname{Nil}(\mathbf{A})$.

Também $\overline{\mathbf{A}}$ é comutativa, logo $[\overline{\mathbf{A}}, \overline{\mathbf{A}}]=(\overline{0})$, isto é $[\mathbf{A}, \mathbf{A}] \subseteq \operatorname{Nil}(\mathbf{A})$, que é nilpotente de índice $s$. Portanto, A satisfaz a identidade

$$
u_{1} \ldots u_{n} \equiv 0
$$


em que cada $u_{i}$ é um comutador ou associador de elementos arbitrários em A. Esta expressão independe de qualquer combinação de parênteses, mesmo que A não seja comutativa ou associativa.

(ii) $\Longrightarrow($ iii): A demonstração é a mesma feita para o caso alternativo.

(iii) $\Longrightarrow$ (i): Seja A uma (-1,1)-álgebra que satisfaz $(x, y, z)^{n} \equiv 0$ e $[x, y]^{n}=0$ para quaisquer $x, y$ e $z$ em A. Comecemos com a identidade $(x, y, z)^{n} \equiv 0$. Como $F_{2}$ é associativa, ela satisfaz, trivialmente esta identidade. Todavia, $F_{2}$ não satisfaz a identidade $[x, y]^{n}=0$, pois esta é uma das identidades que definem variedades não matriciais (no senso de Latshev, Definição 1 ), isto é, se $[x, y]^{n} \equiv 0$ define uma variedade $\mathcal{U}$, então $F_{2} \notin \mathcal{U} \cap$ Assoc (Teorema 2.5.1). Com esta observação, em virtude da Definição 8 , concluímos que $\mathcal{M}$ é não matricial.

$(\mathbf{i}) \Longrightarrow(\mathbf{i v}):$ Seja $\mathbf{A} \in \mathcal{M}$ e suponha que exista $x \in \mathbf{A}$ tal que $x \neq 0$ é nilpotente, para um natural $n$, mas $x \notin \operatorname{Nil}(\mathbf{A})$.

Considere a álgebra quociente $\overline{\mathbf{A}}=\mathbf{A} / \operatorname{Nil}(\mathbf{A}) . \overline{\mathbf{A}}$ também está $\mathcal{M}$, pois $\mathcal{M}$ é variedade. Note que $\overline{0} \neq \bar{x} \in \overline{\mathbf{A}}$, mas $\bar{x}^{n}=\overline{0}$, pois $x^{n}=0$.

Ora, pelo Lema 4.4.4, $\overline{\mathbf{A}}$ é associativa, comutativa, nil semissimples, logo $\overline{\mathbf{A}}$ não tem elementos nilpotentes, isto é,

$$
\bar{x}^{n}=\overline{0} \Longrightarrow \bar{x}=\overline{0} .
$$

Todavia, se $\bar{x}=\overline{0}$, então $x \in \operatorname{Nil}(\mathbf{A})$, uma contradição.

$(\mathbf{i}) \Longrightarrow(\mathbf{v})$ : Seja A uma (-1,1)-álgebra finitamente gerada $\mathcal{M}$ por elementos algébricos em $\mathcal{M}$.

Seja $\mathcal{D}=\mathcal{D}(\mathbf{A})$ o ideal associador de $\mathbf{A}$. O ideal $\mathcal{D}$ é localmente nilpotente (Teorema 4.4.3). Seja $\mathcal{L}$ o radical localmente finito de $\mathbf{A}$.

$$
\mathcal{D} \subseteq \operatorname{Locnil}(\mathbf{A}) \subseteq \mathcal{L}(\mathbf{A}) \Longrightarrow \overline{\mathbf{A}}=\mathbf{A} / \mathcal{L}(\mathbf{A}) \text { é associativa } .
$$

Repetindo as demonstrações já feitas no caso associativo, pois $\overline{\mathbf{A}}$ é associativa finitamente gerada por elementos algébricos numa variedade não matricial $\mathcal{M}$, concluímos $\operatorname{dim}(\overline{\mathbf{A}})<\infty$, isto é, $\overline{\mathbf{A}}=\mathcal{L}(\overline{\mathbf{A}})$. Por outro lado,

$$
\mathcal{L}(\overline{\mathbf{A}})=\mathcal{L}(\mathbf{A} / \mathcal{L}(\mathbf{A}))=(0) .
$$

Logo, concluímos $\overline{\mathbf{A}}=(\overline{0})$, ou seja, $\mathbf{A}=\mathcal{L}(\mathbf{A})$. Portanto, A é de dimensão finita.

Os $\operatorname{casos}(\mathbf{i v}) \Longrightarrow(\mathbf{i}),(\mathbf{v}) \Longrightarrow(\mathbf{i}),(\mathbf{i}) \Longrightarrow(\mathbf{v i}),(\mathbf{v i}) \Longrightarrow(\mathbf{i}),(\mathbf{i}) \Longrightarrow($ vii), (vii) $\Longrightarrow$ $(\mathbf{i}),(\mathbf{i}) \Longrightarrow$ (viii) e (viii) $\Longrightarrow$ (i) são idênticos aos já feitos no caso para álgebras alternativas no Teorema B.

Neste capítulo, exibimos uma caracterização não matricial para álgebras alternativas. Em seguida, generalizamos para uma classe de álgebras alternativas à direita, satisfazendo a identidade cíclica do associador (as álgebras $(-1,1)$, da Seção 4.4). 
Nosso desejo era fornecer uma resposta não matricial para todas as álgebras alternativas à direita, mas não pudemos alcançar este objetivo. A dificuldade principal foi ausência de resultados "satisfatórios" na teoria de radicais para álgebras alternativas à direita ou PI-álgebras alternativas à direita.

Nossos resultados, até agora, foram bem sucedidos quando enfraquecemos a noção de associatividade. Assim, fizemos caracterizações não matriciais para álgebras de Jordan e para álgebras alternativas.

Consideremos agora a identidade comutativa (Exemplo 2.1.7 e Exemplo 2.2.4) e a identidade flexível (Exemplo 2.1.13). Nestes exemplos, observamos que álgebras comutativas são flexíveis. A recíproca é, obviamente, falsa. A identidade flexível, como veremos adiante, pode ser entendida como um tipo de "enfraquecimento" da identidade comutativa.

Nosso próximo passo se dará na tentativa de obter uma caracterização não matricial em uma variedade "larga" o suficiente para conter a variedade das álgebras associativas e a variedade das álgebras comutativas. 
56 ÁLGEBRAS ALTERNATIVAS 


\section{Capítulo 5}

\section{Álgebras de Jordan não-comutativas}

Álgebras de Jordan são álgebras comutativas que satisfazem a identidade de Jordan

$$
\left(x^{2} a\right) x=x^{2}(a x) .
$$

Uma generalização natural destas álgebras para um caso não comutativo é uma classe de álgebras satisfazendo (5.1). A linearização de (5.1) fornece

$$
(x y+y x, a, z)+(y z+z y, a, x)+(z x+x z, a, y)=0,
$$

em que $(x, y, z)$ indica o associador: $(x, y, z)=(x, y) z-x(y z)$. Suponho a característica do corpo de base diferente de 2. Se uma álgebra A satisfaz a identidade (5.2) e contém a unidade 1, então $z=1 \mathrm{em} \mathrm{(5.2)} \mathrm{implica}$

$$
(y, a, x)+(x, a, y)=0
$$

ou, de forma equivalente,

$$
x(a x)=(x a) x
$$

Portanto, uma álgebra unitária A que satisfaz à identidade (5.1) também satisfaz a identidade (5.4), ou seja, A é uma álgebra flexível. Evidentemente, cada álgebra comutativa ou anticomutativa é flexível, portanto a identidade de flexibilidade generaliza tanto comutatividade quanto anticomutatividade.

Desta forma, a seguinte definição será considerada.

Definição 9 (Álgebra de Jordan não comutativa). Uma álgebra de Jordan não comutativa A é uma álgebra satisfazendo as identidades (5.1) e (5.4), ou seja, para quaisquer $x$ e $y \in A$, pode-se afirmar que:

$$
\left(x^{2}, y, x\right)=0 \quad(\text { Jordan })
$$

e

$$
(x, y, x)=0 \quad \text { (Flexível) }
$$

Álgebras de Jordan não comutativas foram introduzidas por Albert in [Alb48] e largamente estudadas in [Sch55, Kok58, Sm71, Sk89, Sh71].

Denotemos a variedade de álgebras de Jordan não comutativas por NCJ. 


\subsection{Exemplos de álgebras em NCJ}

De acordo com a definição 9 as álgebras associativas são, trivialmente, álgebras de Jordan não comutativas. Evidentemente, cada álgebra de Jordan é uma álgebra de Jordan não comutativa. Cada álgebra alternativa $A$ também é uma álgebra de Jordan não comutativa, pois pelo teorema de Artin quaisquer elementos $x, y \in A$ geram uma subálgebra associativa, $\operatorname{logo} A$ satisfaz identidades (5.1) e (5.4). É claro que as álgebras anticomutativas que satisfazem (5.1) também são álgebras de Jordan não comutativas.

Para cada álgebra de Jordan não comutativa $\mathbf{A}$, a álgebra $\mathbf{A}^{(+)}$é de Jordan. Mais geralmente, temos o seguinte resultado:

Lema 5.1.1. Uma álgebra flexível A é álgebra de Jordan não comutativa se, e somente se, a álgebra $\mathbf{A}^{(+)}$é de Jordan.

Este resultado pode ser conferido em [Sch94].

Consideremos agora mais exemplos de álgebras de Jordan não comutativas.

Exemplo 5.1.1 (Álgebras flexíveis quadráticas). Uma álgebra unitária A sobre um corpo $F$ chama-se quadrática sobre $F$ se para cada $a \in \mathbf{A}$ existem elementos $t(a), n(a) \in F$ tais que

$$
a^{2}-t(a) a+n(a) 1=0 .
$$

Lema 5.1.2. Cada álgebra flexivel quadrática A é uma álgebra de Jordan não comutativa.

Demonstração. Basta verificar a identidade (5.1) em A. Pela quadraticidade, nos temos $\left(a^{2}, b, a\right)=t(a)(a, b, a)-n(a)(1, b, a)=0$.

Os exemplos mais importantes de álgebras flexíveis quadráticas são as álgebras obtidas pelo chamado processo de Cayley-Dickson generalizado (veja [Sch94, ZSSS82]). Nos primeiros três passos deste processo se obtêm, começando com os números reais $\mathbb{R}$, o corpo de números complexos $\mathbb{C}$, a álgebra de quatérnios $\mathbb{H}$ e a álgebra de octônios $\mathbb{O}$, que são álgebras de divisão. Se pode continuar este processo de duplicação de álgebras e assim obter as álgebras reais de dimensão $2^{n}$, que não são álgebras de divisão para $n>3$, mas são álgebras flexíveis quadráticas simples, portanto são álgebras de Jordan não comutativas simples. O processo de Cayley-Dickson generalizado está definido para qualquer corpo, portanto estas álgebras existem sobre qualquer corpo.

Lema 5.1.3 (Smith). Seja A uma álgebra flexível quadrática simples sobre um corpo $F$ de característica $\neq 2$. Então a álgebra $\mathbf{A}^{(+)}$é uma álgebra simples de Jordan de tipo Clifford.

Este resultado encontra-se demonstrado em [Sm71].

Exemplo 5.1.4 (Álgebras quase-associativas). Uma álgebra A sobre um corpo $F$ chamase quase-associativa se existir uma extensão quadrática $K$ do corpo $F$ tal que a álgebra $\mathbf{A}_{K}=K \otimes_{F} \mathbf{A}$ é isomorfa a uma álgebra $B(\lambda)$ definida de maneira seguinte: $B$ é uma álgebra associativa sobre $K, \lambda \neq \frac{1}{2}$ é um elemento fixo de $K$, e $B(\lambda)$ tem o mesmo espaço vetorial sobre $K$ como $B$ mas com a multiplicação $*$ definida por

$$
x * y=\lambda x y+(1-\lambda) y x,
$$


para todo $x, y \in B$, onde $x y$ é um produto associativo em $B$.

Cada álgebra quase-associativa é uma álgebra de Jordan não comutativa.

O próximo exemplo foi introduzido em [Kok58] e estudado em [Sk91]).

Exemplo 5.1.5. Sejam $F$ um corpo, $F[X]$ o anel de polinômios sobre o conjunto de variáveis $X=\left\{x_{j} \mid j \in \Lambda\right\}$ e $d=\left\{\partial_{i} \mid i \in \Lambda\right\}$ um sistema de derivação de $F[X]$, em que $\partial_{i}$ são as derivações parciais usuais e $\Lambda$ é um conjunto indexador arbitrário.

Seja $c=\left\{c_{i j} \mid i, j \in \Lambda\right\}$ uma coleção de elementos de $F[X]$ tais que $c_{i j}=-c_{j i}$, para quaisquer $i, j \in \Lambda$. Defina, para elementos arbitrários $f$ e $g$ de $F[X]$ a operação $*$ como a seguir:

$$
f * g:=f g+\sum_{i<j} c_{i j}\left(\frac{\partial f}{\partial x_{i}} \frac{\partial g}{\partial x_{j}}-\frac{\partial g}{\partial x_{j}} \frac{\partial f}{\partial x_{i}}\right) .
$$

A álgebra $(F[X], *)$ é uma álgebra de Jordan não comutativa.

Em [Sh71] encontramos alguns exemplos não triviais de álgebras de Jordan não comutativas. Exibiremos um caso neste trabalho.

Exemplo 5.1.6 (Álgebras semi-alternativas, [Sh71]). Uma álgebra A é dita ser semialternativa se as seguintes identidades são satisfeitas em A:

$$
\begin{aligned}
(x, y, x) & =0 \\
\left(x^{2}, y, x\right) & =0 \\
([x, y], z, z) & =0 .
\end{aligned}
$$

As álgebras semi-alternativas possuem propriedades interessantes. Algumas delas serão exibidas nos próximos resultados.

Lema 5.1.7 (Shestakov, [Sh71]). Seja A uma álgebra semi-alternativa finitamente gerada. Então, sua álgebra adjunta $\mathbf{A}^{(+)}$é finitamente gerada.

Teorema 5.1.8 (Shestakov, [Sh71]). Seja A uma álgebra semi-alternativa arbitrária. Então, A admite um radical localmente nilpotente.

O caso semi-alternativo, citado no exemplo 5.1.6, é um exemplo de álgebra de Jordan não comutativa que nos interessou, pois os resultados do Lema 5.1.7 e do Teorema 5.1.8 serão úteis para uma caracterização não matricial de álgebras de Jordan não comutativas. Deixo o leitor advertido acerca da intenção do nosso trabalho em descrever uma caracterização para variedades não matriciais de álgebras de Jordan não comutativas.

\subsection{1 Álgebras de Jordan não comutativas estritamente primas}

As álgebras de Jordan não comutativas são, em particular, álgebras de potências associativas [Alb48]. Portanto, o conceitos "elemento nilpotente", "álgebra nil" , "ideal nil", "elemento algébrico", "álgebra algébrica" e "ideal algébrico" estão bem definidos nessa classe de álgebras. Em particular, pelos resultados do capítulo 1, os radicais $N i l(A)$ e 
$\operatorname{alg}_{Z}(A)$ estão bem definidos nesta classe de álgebras e são radicais no sentido de AmitsurKurosh.

Porém, o conceito de "álgebra não degenerada" é enfraquecido, pois há diferenças entre o conceito de "divisor de zero absoluto" para álgebras alternativas e de Jordan (ambas são álgebras de Jordan não comutativas).

Uma álgebra de Jordan não comutativa é dita ser prima se é prima no sentido usual de álgebras (Definição 2.3.1.5).

Definição 5.1.9 (Álgebras estritamente primas). Uma álgebra de Jordan não comutativa A é dita ser estritamente prima se A é prima e se a seguinte condição acontece:

$$
I \unlhd \text { A satisfazendo } I \subseteq \operatorname{Degen}\left(\mathbf{A}^{(+)}\right) \Longrightarrow I=(0) .
$$

A definição anterior é uma alternação da noção de "prima não degenerada", como estudado no caso Jordan (Capítulo 3). Note que $\mathbf{A}^{(+)}$é uma álgebra de Jordan, por tanto seu radical degenerado Degen $\left(\mathbf{A}^{(+)}\right)$está, de fato, definido.

O seguinte lema mostra que a classe de álgebras estritamente primas é bastante ampla e inclui todas as álgebras primas nil-semissimples.

Lema 5.1.10. Se A é uma álgebra de Jordan não comutativa prima e Nil-semissimples, então A é estritamente prima.

Demonstração. Se A uma álgebra de Jordan não comutativa prima tal que $\operatorname{Nil}(\mathbf{A})=(0)$. Seja $I$ ideal de $\mathbf{A}$ tal que $I \subseteq \operatorname{Degen}\left(\mathbf{A}^{(+)}\right)$. Como $\mathbf{A}^{(+)}$é de Jordan, por 5.1.1, temos que $\operatorname{Degen}\left(\mathbf{A}^{(+)}\right) \subseteq \operatorname{Nil}\left(\mathbf{A}^{(+)}\right)$. Em outras palavras, $I \subseteq \operatorname{Degen}\left(\mathbf{A}^{(+)}\right) \subseteq \operatorname{Nil}\left(\mathbf{A}^{(+)}\right)$e $I \unlhd A$, tem-se $I \subseteq \operatorname{Nil}(\mathbf{A})$, ou seja, $I$ é um nil ideal de $\mathbf{A}$.

Desta forma, provamos que se $I \subseteq \operatorname{Degen}\left(\mathbf{A}^{(+)}\right)$, então $I \subseteq \operatorname{Nil}(\mathbf{A})$. Por hipótese, $\operatorname{Nil}(\mathbf{A})=(0)$, ou seja,

$$
I \subseteq \operatorname{Nil}(\mathbf{A})=(0) \Longrightarrow I=(0) .
$$

Como $I \subseteq \operatorname{Degen}\left(\mathbf{A}^{(+)}\right)$é tomado de forma arbitrária, concluímos que A é estritamente prima.

Uma maneira de caracterizar as as álgebras de Jordan não comutativas estritamente primas será apresentada nos próximos resultados, que podem ser vistos em [Sk89]. Em particular, o seguinte teorema é um análogo do teorema do Zel'manov sobre a classificação de álgebras primas não degeneradas de Jordan (Teorema 3.2.6).

Teorema 5.1.11 (Skosyrskii). Se A é uma álgebra de Jordan não comutativa estritamente prima, então ela é precisamente de um dos seguintes tipos:

(I) A é comutativa prima não degenerada.

(II) A é prima quase-associativa sobre um centroide estendido.

(III) A é uma ordem central em uma álgebra simples flexível quadrática.

(IV) A álgebra adjunta $\mathbf{A}^{(+)}$é associativa.

Como consequência imediata do Lema 5.1.10, obtemos o seguinte resultado 
Corolário 5.1.12. Seja A uma álgebra de Jordan não comutativa prima Nil-semissimples. Então A satisfaz as condições do Teorema 5.1.11.

Ademais, em [Sk91], podemos encontrar o seguinte resultado, que nos será útil:

Teorema 5.1.13 (Skosyrskii). Seja F um corpo com char $(F) \neq 2$. Toda F-álgebra de Jordan não comutativa A centralmente fechada estritamente prima com álgebra adjunta $\mathbf{A}^{(+)}$associativa é isomorfa a uma subálgebra da F-álgebra $(F(X)$, *) descrita no Exemplo 5.1.5, em que $F(X)$ é a álgebra de funções racionais sobre um conjunto de variáveis $X$.

Neste trabalho, álgebras primas são interessantes por conta da teoria estrutural de radicais em álgebras de Jordan não comutativas, pois estas serão, em certas ocasiões, associativas e comutativas. Na Subseção 2.3.1, estabelecemos que os seguintes radicais estão definidos em uma álgebra de Jordan não comutativa A:

- $\mathrm{Nil}(\mathbf{A})$, o nil radical de A, também conhecido como nil radical superior ou radical de Köethe.

- $\mathfrak{a l g}(\mathbf{A})$, o radical algébrico de $\mathbf{A}$.

Tais resultados se devem ao fato de uma álgebra de Jordan não comutativa ser também uma álgebra de potências associativas.

Relembremos também os seguintes resultados:

Teorema 5.1.2 (Shestakov [ZSh73]). Se A é uma álgebra de potências associativas, então $\mathbf{A} / \mathfrak{a l g}(\mathbf{A})$ é isomorfa a uma soma subdireta de álgebras primas $\mathfrak{a l g}$-semissimples:

$$
\mathbf{A} / \mathfrak{a l g}(\mathbf{A}) \hookrightarrow \prod \mathbf{A}_{\alpha}, \quad \mathbf{A}_{\alpha} \text { é prima e } \mathfrak{a l g}\left(\mathbf{A}_{\alpha}\right)=(0), \forall \alpha .
$$

Essa é a versão do Teorema 2.3.1.17 para álgebras de Jordan não comutativas.

Teorema 5.1.3 (Shestakov [ZSh73]). Se A é uma álgebra de potências associativas, então A/Nil(A) é isomorfa a uma soma subdireta de álgebras primas Nil-semissimples:

$$
\mathbf{A} / \operatorname{Nil}(\mathbf{A}) \hookrightarrow \prod \mathbf{A}_{\alpha}, \quad \mathbf{A}_{\alpha} \text { é prima e } \operatorname{Nil}\left(\mathbf{A}_{\alpha}\right)=(0), \forall \alpha .
$$

A versão do Teorema 2.3.1.18 para álgebras de Jordan não comutativas.

Nos capítulos anteriores, em particular nos casos Jordan e alternativo, estabelecemos a relação entre álgebras primas não degeneradas e variedades não matriciais. No caso alternativo, provamos que álgebras alternativas primitivas numa variedade não matricial são corpos. No caso de Jordan, provamos que álgebras de Jordan primas não degeneradas numa variedade não matricial são dominios associativos e comutativos. Em ambos os casos, temos associatividade e comutatividade para certos tipos de álgebras em variedades não matriciais. Todavia, em ambos os casos, a definição de "não matricialidade" usada (de forma primária ou através de equivalências) é de que álgebras simples em variedades não matriciais são corpos. 
Com a observação anterior e mantendo a definição de "não matricial" para as outras classes de álgebras (Definição 5), é proveitoso o questionamento da seguinte afirmação: uma álgebra estritamente prima $\mathbf{A}$ em uma variedade não matricial $\mathcal{V} \subseteq$ NCJ é comutativa e associativa?

Retornaremos a essa discussão na seção 5.3. Lá serão discutidas e estabelecidas as condições que determinarão resultados em variedades não matriciais de álgebras de Jordan não comutativas.

\section{2 Álgebras de Jordan não comutativas admissíveis}

Em [Sh71], encontramos algumas implicações ou equivalências da identidade flexível, para anéis flexíveis que são $\phi$-operadores, em que $\phi$ é um anel unitário comutativo e associativo com elemento $\frac{1}{2}$ tal que $\frac{1}{2}+\frac{1}{2}=1$, em que 1 é a identidade de $\phi$. Na prática, apenas adequando o objeto à nomenclatura atual, foram estudadas $\phi$-álgebras flexíveis sobre um anel $\phi$ com as características supracitadas. Algumas dessas implicações podem ser formuladas da seguinte forma:

Lema 5.2.1. Em uma $\phi$-álgebra flexível, a identidade $[x \circ y, z]=x \circ[y, z]+[x, z] \circ y$ é válida, em que o é a multiplicação de Jordan: $x \circ y=\frac{1}{2}(x y+y x)$.

Teorema 5.2.2 (Shestakov, [Sh71]). Seja A uma $\phi$-álgebra flexível. Consideremos em A os seguintes conjuntos:

$$
\begin{aligned}
J_{2} & =\left\{\sum_{i} \alpha_{i} a_{i}^{2} \mid \alpha_{i} \in \phi, a_{i} \in \mathbf{A}\right\}, \\
\left(\mathbf{A}^{(+)}\right)^{2} & =\left\{\sum_{i} \alpha_{i}\left(a_{i} \circ b_{i}\right) \mid \alpha_{i} \in F, a_{i}, b_{i} \in \mathbf{A}\right\} .
\end{aligned}
$$

Então $J_{2}=\left(\mathbf{A}^{(+)}\right)^{2}$ é um ideal da $\phi$-álgebra $\mathbf{A}$.

De acordo com o Teorema 5.2.2, a álgebra quociente $\overline{\mathbf{A}}=\mathbf{A} /\left(\mathbf{A}^{(+)}\right)^{2}$ é anticomutativa, isto é, $\bar{a} \circ \bar{b}=0$, para quaisquer $a, b \in \mathbf{A}$.

A partir de agora, a menos que se especifique o contrário, consideraremos álgebras de Jordan não comutativas sobre um corpo $F$ tal que $\operatorname{char}(F) \neq 2$.

Os resultados apresentados em [Sh71] nos motivam a definir, como parte da contribuição inédita desta tese, o seguinte objeto:

Definição 5.2.3 (Álgebra admissível). Diremos que uma álgebra A é admissível se existe um natural $n=n(\mathbf{A})$ tal que a álgebra $\mathbf{A}^{n}$ está contida em $\left(\mathbf{A}^{(+)}\right)^{2}$. O natural $n$ será chamado de índice de admissibilidade da álgebra $\mathbf{A}$.

Definição 5.2.4 (Variedade admissível). Uma variedade $\mathcal{V}$ de álgebras é chamada de admissivel se, para toda álgebra $\mathbf{A}$ em $\mathcal{V}$, existe um natural $n=n(\mathbf{A})$, tal que $\mathbf{A}^{n} \subseteq$ $\left(\mathbf{A}^{(+)}\right)^{2}$. Se o número $n$ acima não depende da álgebra $\mathbf{A} \in \mathcal{V}$, chamaremos $\mathcal{V}$ de $n$ admissivel.

Em outras palavras, uma variedade admissível é uma variedade em que todas as suas álgebras são admissíveis.

Comecemos com o seguinte resultado. 
Lema 5.2.5. Uma variedade $\mathcal{V}$ de álgebras flexíveis é admissível se, e somente se, cada álgebra anticomutativa $\mathbf{A} \in \mathcal{V}$ é nilpotente.

Demonstração. Seja $\mathcal{V}$ uma variedade admissível e seja $\mathbf{A} \in \mathcal{V}$ uma álgebra anticomutativa. Por definição, existe $n=n(\mathbf{A})$ natural tal que $\mathbf{A}^{n} \subseteq\left(\mathbf{A}^{(+)}\right)^{2}$. Ora, $\left(\mathbf{A}^{(+)}, \circ\right)$ é uma zero álgebra, pois A é anticomutativa:

$$
x \circ y=\frac{1}{2} \underbrace{(x y+y x)}_{\mathbf{A} \text { é anticomutativa }}=0 .
$$

Logo, $\left(\mathbf{A}^{(+)}\right)^{2}=(0)$, pelo que concluímos $\mathbf{A}^{n} \subseteq\left(\mathbf{A}^{(+)}\right)^{2}=(0)$, ou seja, A é nilpotente de índice $n$.

A recíproca segue-se desta forma. Seja $\mathbf{A} \in \mathcal{V}$ uma álgebra arbitrária. Como $\mathbf{A}$ é flexível, segue-se que $\left(\mathbf{A}^{(+)}\right)^{2}$ é um ideal de $\mathbf{A}$. Logo, podemos definir a álgebra quociente $\overline{\mathbf{A}}=\mathbf{A} /\left(\mathbf{A}^{(+)}\right)^{2}$ que também está em $\mathcal{V}$, pois $\mathcal{V}$ é variedade. Note, por sua definição, que a álgebra $\overline{\mathbf{A}}$ é anticomutativa. Por hipótese (da condição recíproca), $\overline{\mathbf{A}}$ possui índice de nilpotência $n=n(\overline{\mathbf{A}}), \log \overline{\mathbf{A}}^{n}=(\overline{0})$, ou seja, $\mathbf{A}^{n} \subseteq\left(\mathbf{A}^{(+)}\right)^{2}$.

Salientamos que este resultado é fruto de investigações do autor e do orientador desta tese e será usado mais adiante para obter uma caracterização não matricial.

Seja $\mathcal{V}$ uma variedade admissível. Consideremos a $\mathcal{V}$-álgebra livre $F_{\mathcal{V}}[X]$ sobre um conjunto enumerável de variáveis $X=\left\{x_{\alpha}\right\}$. Pela definição da variedade admissível, $F_{\mathcal{V}}[X]$ possui um índice de admissibilidade, suponhamos $s$. Agora, uma álgebra $\mathbf{A}$ qualquer em $\mathcal{V}$ terá suas identidades determinadas por homomorfismos $\varphi: F_{\mathcal{V}}[X] \rightarrow \mathbf{A}$, via propriedade universal, portanto A também será s-admissível.

Essa observação nos permite enunciar o seguinte resultado:

Lema 5.2.6. Se $\mathcal{V}$ é admissivel, então $\mathcal{V}$ é s-admissivel, em que s é o índice de admissibilidade da álgebra $\mathcal{V}$-livre.

Exemplo 5.2.7. Álgebras associativas são um caso particular das álgebras de Jordan não comutativas. Seja A uma álgebra associativa. Em [ZSSS82], demonstra-se que

$$
\mathbf{A}^{3} \subseteq\left(\mathbf{A}^{(+)}\right)^{2}
$$

Logo, álgebras associativas são 3-admissíveis, ou seja, Assoc é uma variedade 3-admissível de álgebras de Jordan não comutativas.

Exemplo 5.2.8. De forma similar ao caso associativo, álgebras alternativas também são, particularmente, álgebras de Jordan não comutativas. Se A é uma álgebra alternativa, então

$$
\mathbf{A}^{4} \subseteq\left(\mathbf{A}^{(+)}\right)^{2}
$$

Logo, álgebras alternativas são 4-admissíveis ou, de forma similar ao exemplo sobre álgebras associativas, Alt $\subseteq \mathrm{NCJ}$ é uma variedade 4-admissível. Este caso também pode ser visto em [ZSSS82]. 
Os exemplos anteriores mostram que as álgebras associativas são 3-admissíveis e as alternativas são 4-admissíveis. Já era esperado que o índice de admissibilidade de uma álgebra associativa fosse menor ou igual ao de uma álgebra alternativa, pois Assoc $\subseteq$ Alt.

Exemplo 5.2.9. Qualquer álgebra comutativa $\mathbf{A}$ é 2-admissível, pois $\mathbf{A}=\mathbf{A}^{(+)}$.

Exemplo 5.2.10. Uma álgebra anticomutativa A é admissível se, e somente se, A é nilpotente (Lema 5.2.5). Logo, álgebras anticomutativas não nilpotentes não podem ser admissíveis.

A definição de admissibilidade foi considerada como uma resposta a certas condições adequadas para o aspecto "não-matricial" das álgebras de Jordan não comutativas. Veremos, a seguir, como são estabelecidos alguns resultados que nos interessarão mais adiante para obter uma caracterização não matricial. Os seguintes lemas são parte integrante dos resultados originais desta tese.

Lema 5.2.11. Seja $\mathcal{V}$ uma variedade admissível. Se $\mathbf{A} \in \mathcal{V}$ é uma álgebra finitamente gerada, então $\mathbf{A}^{(+)}$também é finitamente gerada.

Demonstração. É suficiente demonstrar o caso em que $\mathbf{A}$ é uma álgebra $\mathcal{V}$ livre. Seja $\mathbf{A}=F_{\mathcal{V}}\left[x_{1}, \cdots, x_{k}\right]$ a álgebra $\mathcal{V}$-livre finitamente gerada pelas variáveis $x_{1}, \cdots x_{k}$. Por hipótese, A é admissível, isto é, existe um natural $m$ tal que $\mathbf{A}^{m} \subseteq\left(\mathbf{A}^{(+)}\right)^{2}$. Seja $Y=$ $\{v \in \mathbf{A} \mid v$ é monômio $\operatorname{deg}(v) \leq m\}$.

Mostraremos que $\mathbf{A}^{(+)}=$o-alg $\langle Y\rangle$, ou seja, $\mathbf{A}^{(+)}$é gerada como $F$-álgebra pelo conjunto $Y$ através da operação o. A inclusão o-alg $\langle Y\rangle \subseteq \mathbf{A}^{(+)}$é evidente. Provemos a inclusão contrária.

Reduziremos este problema aos monômios de A. Provaremos que cada monômio (nas variáveis $\left.x_{1}, \cdots, x_{k}\right)$ pode ser expresso através de elementos de $Y$ com a operação o. Façamos por indução sobre $\operatorname{deg}(v)$. Seja $v$ um monômio arbitrário em A. Se $\operatorname{deg}(v) \leq m$, então, por construção, $v \in Y$. Apliquemos o passo indutivo, $\operatorname{supondo} \operatorname{deg}(v)>m$. Ora, $\mathbf{A}^{m} \subseteq\left(\mathbf{A}^{(+)}\right)^{2}$ e isto implica $v \in\left(\mathbf{A}^{(+)}\right)^{2}$. Portanto, $v=\sum u_{i} \circ w_{i}$, cujos monômios $u_{i}$ e $w_{i}$ são tais que $\operatorname{deg}\left(u_{i}\right)<\operatorname{deg}(v)$ e $\operatorname{deg}\left(w_{i}\right)<\operatorname{deg}(v)$, para todo $i$, ou seja, como $\operatorname{deg}(v)>m$, podemos reescrever esta passagem da seguinte forma: $\operatorname{deg}\left(u_{i}\right) \leq m$ e $\operatorname{deg}\left(w_{i}\right) \leq m$, para todo $i$. Por hipótese indutiva, cada $u_{i}$ e cada $w_{i}$ está em $Y$. Logo, $v \in$ o-alg $\langle Y\rangle$. Como $v$ é um monômio arbitrário nas finitas variáveis $x_{1}, \cdots, x_{k}$, segue-se que $\mathbf{A}^{(+)}=0$-alg $\langle Y\rangle$, ou seja, $\mathbf{A}^{(+)}$é uma $F$-álgebra finitamente o-gerada.

Se $B \in \mathcal{V}$ é uma álgebra finitamente gerada por $k$ elementos $a_{1}, \ldots, a_{k}$, existe um único homomorfismo $\varphi_{B}: \mathbf{A} \rightarrow B$, via propriedade universal, tal que $\varphi\left(x_{i}\right)=a_{i}, i=1, \ldots, k$. O homomorfismo induzido $\varphi_{B}^{+}: \mathbf{A}^{(+)} \rightarrow B^{(+)}$determina, seguindo as mesmas técnicas, que a álgebra $B^{(+)}$é gerada pela imagem homomórfica $\varphi(Y)$ do conjunto $Y$, portanto é finitamente gerada.

Lema 5.2.12. Seja $\mathcal{V} \subseteq \mathrm{NCJ}$ uma variedade $m$-admissivel. Sejam $\mathbf{A} \in \mathcal{V}, I \unlhd \mathbf{A} e$ $M \subseteq \mathbf{A}$ um subespaço tal que $I \circ \mathbf{A} \subseteq M$. Então, para quaisquer $a_{1}, \cdots, a_{m} \in \mathbf{A}$, tem-se

$$
I T_{a_{1}} \cdots T_{a_{m-1}} \subseteq M, \quad T_{a_{j}} \in\left\{L_{a}, R_{a}\right\}
$$

Em outras palavras, $I\left(T_{\mathbf{A}}\right)^{m-1} \subseteq M$. 
Demonstração. Consideremos a álgebra $\mathcal{V}$-livre $\mathcal{F}=F_{\mathcal{V}}[X]$. Por hipótese, $\mathcal{F}$ é $m$-admissível, $\mathcal{F}^{m} \subseteq \mathcal{F} \circ \mathcal{F}$, então para as variáveis livres $x_{0}, x_{1}, \cdots, x_{m}$ nós temos

$$
x_{0} T_{x_{1}} \cdots T_{x_{m}}=\sum_{i} u_{i} \circ v_{i}
$$

em que $u_{i}$ e $v_{i}$ são monômios em $x_{0}, x_{1} \cdots, x_{m-1}$, tais que para cada índice $i$ ou $x_{0} \in u_{i}$ ou $x_{0} \in v_{i}$. Seja $b \in I$ um elemento arbitrário. Consideremos um homomorfismo $\varphi: \mathcal{F} \rightarrow \mathbf{A}$, tal que $\varphi\left(x_{0}\right)=b, \varphi\left(x_{i}\right)=a_{i}$, para $1 \leq i \leq m$. Então, temos

$$
b T_{a_{1}} \cdots T_{a_{m}} \in I \circ \mathbf{A} \subseteq M .
$$

Como $b \in I$ é arbitrário, o resultado está provado.

O seguinte lema é muito útil e pode ser encontrado em [Sh71]. Importante salientar que, neste caso, o próximo lema não é de autoria do elaborador da tese.

Lema 5.2.13. Seja A uma álgebra arbitrária. Se $v \in \mathbf{A}^{2^{n}}$ é um elemento arbitrário, então $v$ é uma combinação linear de elementos do tipo $a_{1} T_{a_{2}} \cdots T_{a_{n}}$, em que $a_{i} \in \mathbf{A} e$ $T_{x} \in\left\{L_{x}, R_{x}\right\}$.

Demonstração. Seja $a \in \mathbf{A}^{2^{n}}$.

$$
\begin{aligned}
a & =\sum \underbrace{a_{1}}_{a_{1} \in \mathbf{A}^{2^{n-1}}} a_{2}=\sum \underbrace{a_{1}}_{a_{1} \in \mathbf{A}^{2^{n-1}}} R_{a_{2}}=\sum \underbrace{a_{11} a_{12}}_{a_{12} \in \mathbf{A}^{2^{n-2}}} R_{a_{2}}= \\
& =\sum \underbrace{a_{11}}_{a_{11} \in \mathbf{A}^{2^{n-2}}} a_{12} R_{a_{2}}=\sum \underbrace{a_{12}}_{a_{12} \in \mathbf{A}^{2^{n-2}}} L_{a_{11}} R_{a_{2}}=\cdots=\sum b_{1} \underbrace{T_{b_{2}} \cdots T_{b_{n}}}_{(n-1) \text { operadores }},
\end{aligned}
$$

em que $T_{b_{j}} \in\left\{R_{b_{j}}, L_{b_{j}}\right\}$.

A proposição a seguir é uma contribuição original do autor da tese.

Proposição 5.2.14. Seja $\mathbf{A} \in \mathcal{V}$, em que $\mathcal{V}$ é m-admissivel. Então, para todo natural $n$, existe uma função $f(n)$ tal que $\mathbf{A}^{2^{f(n)}} \subseteq\left(\mathbf{A}^{(+)}\right)^{n}$.

Demonstração. Façamos por indução sobre $n$. É claro que $\mathbf{A}=\mathbf{A}^{(+)}, \operatorname{logo} f(1)=0$. Como $\mathcal{V}$ é $m$-admissível, $\mathbf{A}^{m} \subseteq\left(\mathbf{A}^{(+)}\right)^{2}$, logo $f(2)=\left[\log _{2} m\right]+1$. Por hipótese indutiva, admita que $f(n-1)$ já esteja determinado. Provemos que $f(n)=f(n-1)+(m-1)$ atende o passo indutivo.

Seja $a \in \mathbf{A}^{2^{f(n-1)+(m-1)}}$. Pelo Lema 5.2.13,

$$
a=a_{1} \underbrace{T_{a_{2}} \cdots T_{a_{f(n-1)}}}_{f(n-1)-1 \text { operadores }} T_{a_{f(n-1)+1}} \cdots T_{a_{f(n-1)+(m-1)}},
$$

em que $T_{a_{j}} \in\left\{R_{a_{j}}, L_{a_{j}}\right\}$ e $a_{i} \in \mathbf{A}$.

Por hipótese indutiva, $a_{1} T_{a_{2}} \cdots T_{a_{f(n-1)}} \in \mathbf{A}^{f(n-1)} \subseteq\left(\mathbf{A}^{(+)}\right)^{n-1}$. Sejam $I=\mathbf{A}^{f(n-1)} \unlhd$ A e $M=\left(\mathbf{A}^{(+)}\right)^{n} \subseteq \mathbf{A}$ subespaço. Então

$$
I \circ A=\mathbf{A}^{f(n-1)} \circ A \subseteq\left(\mathbf{A}^{(+)}\right)^{n-1} \circ A \subseteq\left(\mathbf{A}^{(+)}\right)^{n}=M .
$$


Logo, $I\left(T_{\mathbf{A}}\right)^{m-1} \subseteq M$ e, pelo Lema 5.2.12, segue-se que

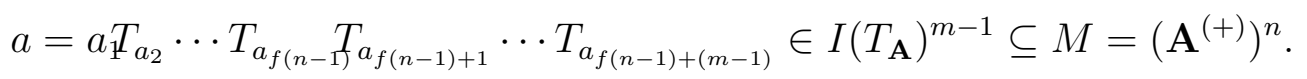

Vamos agora introduzir o conceito de NJPI-álgebra e de variedade essencial para álgebras de Jordan não comutativas.

Definição 5.2.15 (NJPI-álgebra). Diremos que uma álgebra de Jordan não comutativa A é uma $N J P I$-álgebra se a álgebra $\mathbf{A}^{(+)}$é uma Jordan PI-álgebra. Chamemos uma variedade $\mathcal{V}$ de álgebras de Jordan não comutativas de essencial se a álgebra $\mathcal{V}$-livre $F_{\mathcal{V}}[X]$ de posto enumerável é uma $N J P I$-álgebra.

Evidentemente, se $\mathcal{V}$ é uma variedade essencial de álgebras de Jordan não comutativas então cada álgebra do conjunto $\mathcal{V}^{(+)}=\left\{\mathbf{A}^{(+)} \mid \mathbf{A} \in \mathcal{V}\right\}$ satisfaz as identidades polinomiais da $P I$-álgebra de Jordan $F_{\mathcal{V}}[X]^{(+)}$e logo é uma $P I$-álgebra de Jordan. Em particular, cada álgebra comutativa de $\mathcal{V}$ é uma $P I$-álgebra de Jordan. É fácil verificar também que cada álgebra associativa em $\mathcal{V}$ é uma $P I$-álgebra (associativa).

Porém, a seguinte questão está em aberto.

Questão 5.2.1. Seja $\mathcal{V}$ uma variedade de álgebras de Jordan não comutativas onde cada álgebra comutativa é uma $P I$-álgebra de Jordan e cada álgebra associativa é uma $P I$ álgebra (associativa). Será que $\mathcal{V}$ é essencial?

As construções usadas até aqui serão usadas na obtenção do seguinte resultado, também inédito na literatura.

Teorema 5.2.16. Seja $\mathcal{V}$ uma variedade essencial m-admissível de álgebras de Jordan não comutativas. Se $\mathbf{A} \in \mathcal{V}$ é uma álgebra finitamente gerada, então $\mathrm{Nil}(\mathbf{A})$ é nilpotente.

Demonstração. Seja $\mathbf{A} \in \mathcal{V}$ uma álgebra de Jordan não comutativa finitamente gerada. Como $\mathcal{V}$ é essencial, a álgebra $\mathbf{A}^{(+)}$é uma $P I$-álgebra de Jordan. Pelo Lema 5.2.11, $\mathbf{A}^{(+)}$é uma PI-álgebra de Jordan finitamente gerada. Pelo Teorema 2.3, $\operatorname{Nil}\left(\mathbf{A}^{(+)}\right)$é nilpotente.

Seja $s$ natural tal que $\operatorname{Nil}\left(\mathbf{A}^{(+)}\right)^{s}=(0)$. É sabido, de forma geral, que $(\operatorname{Nil}(\mathbf{A}))^{(+)} \subseteq$ $\operatorname{Nil}\left(\mathbf{A}^{(+)}\right)$. Pela Proposição 5.2.14, para o natural $s$ supracitado, existe $f(s)$ tal que

$$
(\operatorname{Nil}(\mathbf{A}))^{2^{f(s)}} \subseteq\left((\operatorname{Nil}(\mathbf{A}))^{(+)}\right)^{s} \subseteq\left(\operatorname{Nil}\left(\mathbf{A}^{(+)}\right)\right)^{s}=(0) .
$$

Portanto, Nil(A) é nilpotente.

Seguindo [Sh71], o seguinte resultado é válido:

Teorema 5.2.17. Se $\mathcal{V} \subseteq$ NCJ é uma variedade admissivel, então o radical localmente nilpotente pode ser definido em qualquer álgebra de $\mathcal{V}$.

Em seguida, temos mais um resultado original (desta tese).

Teorema 5.2.18. Se $\mathcal{V} \subseteq$ NCJ é uma variedade m-admissivel, então o radical localmente finito pode ser definido em qualquer álgebra de $\mathcal{V}$. 
Demonstração. Vamos seguir um esquema padrão. Mostraremos que, se $\mathbf{A} \in \mathcal{V}$ é finitamente gerada e $I \unlhd \mathbf{A}$ é tal que $I$ e $\mathbf{A} / I$ são localmente finitas, então $\mathbf{A}$ é de dimensão finita.

Consideremos a álgebra adjunta $\mathbf{A}^{(+)}$, que é de Jordan e, portanto, já possui o radical localmente finito $\mathcal{L}\left(\mathbf{A}^{(+)}\right)$. Como $\mathcal{V}$ é $m$-admissível, então $\mathbf{A}^{(+)}$é finitamente gerada também (Lema 5.2.11). O ideal $I^{(+)} \unlhd \mathbf{A}^{(+)}$e a álgebra $(\mathbf{A} / I)^{(+)} \cong \mathbf{A}^{(+)} / I^{(+)}$também são localmente finitos, portanto $I^{(+)} \subseteq \mathcal{L}\left(\mathbf{A}^{(+)}\right)$e $\mathcal{L}\left(\mathbf{A}^{(+)} / I^{(+)}\right)=\mathbf{A}^{(+)} / I^{(+)}$. Ademais

$$
\mathbf{A}^{(+)} / \mathcal{L}\left(\mathbf{A}^{(+)}\right) \cong \frac{\mathbf{A}^{(+)} / I^{(+)}}{\mathcal{L}\left(\mathbf{A}^{(+)}\right) / I^{(+)}}
$$

é uma imagem homomórfica de $\mathbf{A}^{(+)} / I^{(+)}$, portanto também é localmente finita, ou seja,

$$
\mathbf{A}^{(+)} / \mathcal{L}\left(\mathbf{A}^{(+)}\right)=\mathcal{L}\left(\mathbf{A}^{(+)} / \mathcal{L}\left(\mathbf{A}^{(+)}\right)\right)=(0) .
$$

Pelo que concluímos que $\mathbf{A}^{(+)}=\mathcal{L}\left(\mathbf{A}^{(+)}\right)$, isto é, $\mathbf{A}^{(+)}$é localmente finita. Mas $\mathbf{A}^{(+)}$é finitamente gerada, portanto de dimensão finita, ou seja, $\operatorname{dim} \mathbf{A}=\operatorname{dim} \mathbf{A}^{(+)}<\infty$.

Nosso próximo passo é tratar das variedades não matriciais.

\subsection{Variedades não matriciais em NCJ}

A subseção 5.1 .1 foi encerrada com o seguinte questionamento: uma álgebra estritamente prima $\mathbf{A}$ em uma variedade não matricial $\mathcal{V} \subseteq \mathrm{NCJ}$ é comutativa e associativa?

A resposta é aparentemente positiva, mas primeiramente precisamos definir o que é "ser não matricial" no caso de álgebras de Jordan não comutativas.

Seja $\mathcal{V}$ uma variedade de $F$-álgebras, nos casos especificados a seguir. Retomando a discussão já feita em torno da definição de variedade não matricial, percebemos que Latyshev começa com uma noção mais objetiva, no caso associativo: uma variedade $\mathcal{V} \subseteq$ Assoc é não matricial se $F_{2} \notin \mathcal{V}$. Para o caso de álgebras alternativas, nós mantivemos a definição segundo a proposta de Latyshev: uma variedade $\mathcal{V} \subseteq$ Alt é não matricial se $F_{2} \notin \mathcal{V}$ (em ambos os casos, $F_{2}$ está munido da multiplicação usual de matrizes). Para as álgebras de Jordan, adaptamos essa condição: uma variedade $\mathcal{V} \subseteq$ Jord é não matricial se $\left(\mathcal{H}_{2}, \circ\right) \notin \mathcal{V}$.

Nessas classes de álgebra, nós provamos que tais asserções não matriciais equivaliam à seguinte condição: uma variedade $\mathcal{V}$ é não matricial se qualquer álgebra simples (dentro dela) é um corpo. Em outras palavras, estendemos a noção de "variedade não matricial": em vez de procurarmos um 'tipo matricial' de álgebra que não deve pertencer à variedade (talvez esse tipo matricial não possa ser estabelecido trivialmente), estabelecemos o critério "se é simples, então é corpo" como uma forma de caracterizar tais variedades.

Fixemos, por ora, esta noção de variedade não matricial: uma variedade $\mathcal{V}$ é não matricial se álgebras simples em $\mathcal{V}$ são corpos.

O próximo exemplo mostra que a noção de "ser não matricial", como estabelecida acima, pode não estar bem definida para álgebras de Jordan não comutativas.

Exemplo 5.3.1. Considere a álgebra $\mathbf{A}=(\mathbb{C}[x, y], *)$, com $c_{12}=-c_{21}=x$ (tome como referência o Exemplo 5.1.5), em que $\mathbb{C}[x, y]$ é o anel de polinômios de duas variáveis sobre 
o corpo dos complexos $\mathbb{C}$ e a multiplicação $*$ é definida da seguinte forma:

$$
f * g=f g+x\left(f_{x}^{\prime} g_{y}^{\prime}-f_{y}^{\prime} g_{x}^{\prime}\right) .
$$

Tal álgebra é similar ao Exemplo 5.1.5, isto é, ela é uma álgebra de Jordan não comutativa.

A respeito dessa álgebra, pode-se afirmar que:

- cada imagem homomórfica simples de A é um corpo (isomorfo a $\mathbb{C}$ ).

- $\operatorname{Nil}(\mathbf{A})=(0): \mathbf{A}$ é prima, pois $\mathbf{A}^{(+)} \cong \mathbb{C}[x, y]$ é um domínio.

- A não é associativa nem comutativa.

Não pudemos provar que toda álgebra simples em $\operatorname{var}(\mathbf{A})$, a variedade gerada por $\mathbf{A}$, é um corpo, mas conjecturamos assim. E se a resposta for positiva, teremos uma variedade não matricial que não satisfaz a identidade $[x, y]^{n}=0$ : isso é algo bem diferente das propriedades de variedades não-matriciais nos casos já considerados.

Desta forma, adotaremos parte da tese da questão feita no início desta seção como definição para variedades não matriciais de álgebras de Jordan não comutativas:

Definição 10. Uma variedade $\mathcal{V}$ de álgebras de Jordan não comutativas é dita ser não matricial se qualquer álgebra prima Nil-semissimples $\mathbf{A} \in \mathcal{V}$ é comutativa e associativa.

Observação 5.3.2. A Definição 10 deixa evidentes as seguintes afirmações.

M1) Se $\mathcal{V} \subseteq$ NCJ é não matricial, então $F_{2} \notin \mathcal{V}$ e $\mathcal{H}_{2} \notin \mathcal{V}$.

De fato, $F_{2}$ e $\mathcal{H}_{2}$ são casos particulares de álgebras de Jordan não comutativas. $F_{2}$ é simples, Nil-semissimples e associativa, mas não é comutativa. Similarmente, $\mathcal{H}_{2}$ simples, Nil-semissimples e comutativa, mas não é associativa.

M2) Se $\mathcal{V} \subseteq$ NCJ é tal que $F_{2} \in \mathcal{V}$ ou $\mathcal{H}_{2} \in \mathcal{V}$, então $\mathcal{V}$ não é uma variedade de álgebras de Jordan não comutativas não matricial.

A condição M2 é, tão somente, a contraposição de M1.

Os próximos resultados, a menos que especifiquemos o contrário, são inéditos e compõem a originalidade desta tese.

Em virtude da Observação 5.3.2, consideremos a seguinte proposição:

Proposição 5.3.3. Seja $\mathcal{V} \subseteq$ NCJ uma variedade. Considere as seguintes condições:

(NM1) Qualquer álgebra prima Nil-semissimples em $\mathcal{V}$ é comutativa e associativa.

(NM2) Qualquer álgebra simples em $\mathcal{V}$ é corpo.

(NM3) $F_{2} \notin \mathcal{V}$ e $\mathcal{H}_{2} \notin \mathcal{V}$.

Então nós temos as seguintes relações entre as condições (NM1), (NM2), (NM3):

$$
\begin{aligned}
& (N M 1) \Rightarrow(N M 3),(N M 2) \Rightarrow(N M 3), \\
& (N M 3) \nRightarrow(N M 1), \quad(N M 3) \nRightarrow(N M 2), \\
& (N M 1) \not \Rightarrow(N M 2) .
\end{aligned}
$$


Demonstração. Já vimos que $(N M 1) \Rightarrow(N M 3)$. Evidentemente, também $(N M 2) \Rightarrow$ $(N M 3)$. Tomando $\mathcal{V}=$ Lie, a variedade de álgebras de Lie, observamos que Lie satisfaz (NM3) - pois álgebras de Lie são anticomutativas - e (NM1) - observe, mais uma vez, a anticomutatividade em Lie -, mas não satisfaz $(N M 2)$ - corpos são álgebras comutativas. Finalmente, consideremos a álgebra $A=(F[X], *)$ do exemplo 5.1 .5 com pelo menos um $c_{i j} \neq 0$. Então, na variedade $\mathcal{V}=\operatorname{var}(A)$ todas as álgebras satisfazem a identidade $(x, y, z)_{+}=0$, portanto $F_{2}, \mathcal{H}_{2} \notin \mathcal{V}$ e $\mathcal{V}$ satisfaz $(N M 3)$. A álgebra $A$ é simples, pois $A^{(+)}$é um corpo, mas não é um corpo, pois nem é comutativa, portanto $\mathcal{V}$ não satisfaz $(N M 2)$ nem $(N M 1)$.

Deixamos em aberto a seguinte questão:

Questão 5.3.4. É certo que $(N M 2) \Rightarrow(N M 1)$ ?

Procedemos agora com propriedades de variedades não matriciais de álgebras de Jordan não comutativas.

Lema 5.3.5. Seja $\mathcal{V}$ uma variedade não matricial de álgebras de Jordan não comutativas. Então as seguintes identidades verificam em $\mathcal{V}$ para certos $n, m$ naturais:

$$
(x, y, z)^{n}=0
$$

$e$

$$
[x, y]^{m}=0 .
$$

Demonstração. Seja $F_{\mathcal{V}}[X]$ a álgebra livre em $\mathcal{V}$ de um conjunto de geradores livres $X$ $\operatorname{com}|X| \geq 3$. Seja $N$ o nil radical de $F_{\mathcal{V}}[X]$. Segue-se, do Teorema 5.1.3, que

$$
F_{\mathcal{V}}[X] / N \hookrightarrow \prod_{\alpha} \mathbf{A}_{\alpha}
$$

em que cada $\mathbf{A}_{\alpha}$ é prima Nil-semissimples. Como $\mathcal{V}$ é não matricial, cada $\mathbf{A}_{\alpha}$ é associativa e comutativa, isto é, $\overline{F_{\mathcal{V}}[X]}=F_{\mathcal{V}}[X] / N$ é associativa e comutativa.

Sejam $x, y, z \in X$ e sejam $\bar{x}, \bar{y}$ e $\bar{z}$ suas respectivas imagens homomórficas através do epimorfismo $F_{\mathcal{V}}[X] \rightarrow F_{\mathcal{V}}[X] / N$. Pelo resultado acima,

$$
(\bar{x}, \bar{y}, \bar{z})=(\overline{0})
$$

$\mathrm{e}$

$$
[\bar{x}, \bar{y}]=(\overline{0})
$$

Em outras palavras,

$$
(x, y, z) \in N
$$

e

$$
[x, y] \in N .
$$

Logo, existe $n$ tal que $(x, y, z)^{n}=0$ e existe $m$ tal que $[x, y]^{m}=0$.

Pela propriedade universal de álgebra universal, nós temos que em qualquer álgebra $A \in \mathcal{V}$, para quaisquer elementos $a, b, c \in A$, se verificam também $(a, b, c)^{n}=0,[a, b]^{m}=$ 0 .

O seguinte resultado nos será útil. 
Proposição 5.3.6. Uma variedade não matricial $\mathcal{V}$ de álgebras de Jordan não comutativas é essencial. Ademais, o conjunto $\mathcal{V}^{(+)}$gera uma variedade não matricial de álgebras de Jordan.

Demonstração. Seja $\mathbf{A}_{\mathcal{V}}[X]$ álgebra de Jordan não comutativa livre de $\mathcal{V}$ e sejam $x, y, z \in$ $X$ elementos arbitrários. Pelo Lema 5.3.5, associadores e comutadores formados por quaisquer elementos de $\mathbf{A}_{\mathcal{V}}[X]$ estão em $\operatorname{Nil}\left(\mathbf{A}_{\mathcal{V}}[X]\right)$. Seja $(x, y, z)_{+}$o associador da álgebra $\left(\mathbf{A}_{\mathcal{V}}[X]\right)^{(+)}$. Já é sabido (Lema 2.1.17) que

$$
4(x, y, z)_{+}=\underbrace{(x, y, z)-(z, y, x)+(y, x, z)-(z, x, y)+(x, z, y)-(y, z, x)+[y,[x, z]]}_{\text {cada parcela está em } \operatorname{Nil}\left(\mathbf{A}_{\mathcal{V}}[X]\right)} .
$$

Logo, $(x, y, z)_{+}$está em $\operatorname{Nil}\left(\mathbf{A}_{\mathcal{V}}[X]\right)$. Portanto, existe $n$ natural tal que $(x, y, z)_{+}^{n}=0$. Agora seja $J \in \mathcal{V}^{(+)}$escolhida arbitrariamente. Existe $B \in \mathcal{V}$ tal que $J=B^{(+)}$. Sejam $a, b, c \in J$ elementos arbitrários. Observe que $J=B^{(+)}=B$ (enquanto conjuntos). Considere o homomorfismo

$$
\varphi: \mathbf{A}_{\mathcal{V}}[X] \rightarrow B
$$

em que $\varphi(x)=a, \varphi(y)=b$ e $\varphi(z)=c$. Dessa forma,

$$
\varphi\left((x, y, z)_{+}\right)=(a, b, c)_{+},
$$

implicando em

$$
0=\varphi(0)=\varphi\left((x, y, z)_{+}^{n}\right)=(a, b, c)_{+}^{n} .
$$

Como $J \in \mathcal{V}^{(+)}$é tomada de forma arbitrária, segue-se que toda álgebra em $\mathcal{V}^{(+)}$satisfaz a identidade $(s, t, u)^{n} \equiv 0$ (o associador agora é descrito a partir da multiplicação em $J$ ).

Todavia,

$$
(s, t, u)^{n} \equiv 0
$$

é uma identidade não trivial em Jord, portanto $\mathcal{V}^{(+)}$está contido na variedade definida pela esta identidade. Pelo Teorema A, a identidade (5.9) define, precisamente, variedades não matriciais em Jord, ou seja, $\mathcal{V}^{(+)}$está contido numa variedade não matricial de álgebras de Jordan.

Demonstremos a generalização do Teorema 2 para o caso das álgebras de Jordan não comutativas. Ressaltamos que este teorema é um resultado original desta tese.

Teorema D. Seja $\mathcal{M}$ uma variedade $m$-admissivel de álgebras de Jordan não comutativas sobre um corpo infinito $F$ de caracteristica diferente de 2.

As seguintes condições são equivalentes:

i) $\mathcal{M}$ é não matricial.

ii) Seja $\mathbf{A} \in \mathcal{M}$ uma álgebra finitamente gerada. Para algum s, A satisfaz a identidade

$$
u_{1} u_{2} \ldots u_{n} \equiv 0
$$

em que cada $u_{i}$ ou é comutador ou é associador de elementos arbitrários em $\mathbf{A}$. $O$ produto da identidade (5.10) independe de uma combinação de parênteses, mesmo que A seja não associativa. 
iii) Para qualquer $\mathbf{A} \in \mathcal{M}$, existe $m$ tal que $\mathbf{A}$ satisfaz $(x, y, z)^{m} \equiv 0$ e $[x, y]^{m} \equiv 0$, para quaisquer elementos $x, y, z \in \mathbf{A}$.

Além disso, as condições equivalentes (i) a (iii) implicam no seguinte:

iv) Para qualquer $A \in \mathcal{M}$, os elementos nilpotentes de $\mathbf{A}$ formam um ideal.

v) Se $A \in \mathcal{M}$ e $a \in A$ é nilpotente, então o ideal bilateral gerado por a é nil.

vi) Se $A \in \mathcal{M}$ e $a \in A$ é nilpotente, então o ideal à esquerda (ou o ideal à direita) gerado por a é nil.

vii) Se $A \in \mathcal{M}$ e $a, b \in A$ são elementos nilpotentes, então $a+b$ também é nilpotente.

viii) Seja $A \in \mathcal{M}$. Qualquer conjunto finito $\left\{a_{1}, a_{2}, \cdots a_{k}\right\}$ de elementos algébricos gera uma álgebra de dimensão finita $A_{0} \subseteq A$.

Demonstração. Vamos observar as condições do teorema e estabelecer as devidas equivalências.

(i) $\Longrightarrow$ (ii): Seja A uma álgebra de Jordan não comutativa finitamente gerada em $\mathcal{M}$ e seja $\operatorname{Nil}(\mathbf{A})$ o seu nil radical.

Note que $\mathcal{M} \subseteq$ NCJ é não matricial e $m$-admissível, logo, pela Proposição 5.3.6, $\mathcal{M}$ é essencial $m$-admissível. O Teorema 5.2.16 nos assegura que Nil(A) é nilpotente, suponhamos de índice $s$.

Pelo Lema 5.3.5, $\overline{\mathbf{A}}=\mathbf{A} / \operatorname{Nil}(\mathbf{A})$ é associativa e comutativa, isto é,

$$
(\overline{\mathbf{A}}, \overline{\mathbf{A}}, \overline{\mathbf{A}})=(\overline{0})
$$

e

$$
[\overline{\mathbf{A}}, \overline{\mathbf{A}}]=(\overline{0}) .
$$

Isso implica que $(\mathbf{A}, \mathbf{A}, \mathbf{A}) \subseteq \operatorname{Nil}(\mathbf{A})$ e $[\mathbf{A}, \mathbf{A}] \subseteq \operatorname{Nil}(\mathbf{A})$, ou seja, adotando o mesmo estilo do Lema 5.3.5, associadores e comutadores definidos em quaisquer elementos de $\mathbf{A}$ estão em $\operatorname{Nil}(\mathbf{A})$.

Como Nil(A) é nilpotente, A satisfaz a identidade

$$
u_{1} u_{2} \ldots u_{s} \equiv 0
$$

em que cada $u_{i}$ é um comutador ou um associador.

A identidade 5.10 independe de qualquer combinação de parênteses entre os elementos $u_{i}$, mesmo que $\mathbf{A}$ não seja comutativa ou associativa.

(ii) $\Longrightarrow$ (iii): Observe que esta parte é muito parecida com o Lema 5.3.5. Seja $\mathbf{A}_{\mathcal{M}}=$ $F_{\mathcal{M}}[x, y, z]$ a álgebra $\mathcal{M}$-livre finitamente gerada por 3 elementos. Por (ii), ela satisfaz (5.10) para um inteiro fixado $m=s$. Em particular, $(x, y, z)^{m}=0$ ou $[u, v]^{m}=0$ para os geradores $x, y, z \in J_{\mathcal{M}}$, $\operatorname{com} u, v \in\{x, y, z\}$. Seja $\mathbf{A} \in \mathcal{M}$ uma álgebra de Jordan não comutativa arbitrária e sejam $a, b, c \in \mathbf{A}$. Pela propriedade universal das álgebras livres, a aplicação $x \mapsto a, y \mapsto b, z \mapsto c$ define um único homomorfismo $\varphi: \mathbf{A}_{\mathcal{M}} \rightarrow \mathbf{A}$. Tem-se

$$
0=\varphi(0)=\varphi\left((x, y, z)^{s}\right)=(\varphi(x), \varphi(y), \varphi(z))^{s}=(a, b, c)^{s}
$$


$\mathrm{e}$

$$
0=\varphi(0)=\varphi\left([u, v]^{s}\right)=[\varphi(u), \varphi(v)]^{s}=[a, b]^{s} .
$$

Portanto as identidades $(x, y, z)^{m}=0 \mathrm{e}[x, y]^{m}=0$ são satisfeitas por todas as álgebras em $\mathcal{M}$.

(iii) $\Longrightarrow(\mathbf{i})$ : Seja $\mathcal{M}$ uma variedade e suponha que qualquer álgebra em $\mathcal{M}$ satisfaça a identidade $(x, y, z)^{m}=[x, y]^{m}=0$. Seja $\mathbf{A} \in \mathcal{M}$ uma álgebra prima e Nil-semissmples. Pelo Lema 5.1.10, A é estritamente prima. Sendo assim, temos os casos a seguir, em virtude do Teorema 5.1.11:

1. A é comutativa prima não degenerada. Nesse caso, A é álgebra de Jordan prima não degenerada, logo (pela Proposição 3.3.1) é associativa e comutativa.

2. A é quase-associativa sobre um centroide estendido. Como $\mathcal{M}$ é homogênea, podemos supor, sem perda, que $\mathbf{A}=B^{(\lambda)}$, com $\lambda \neq 1 / 2$, em que $B$ é álgebra associativa. Ora, os ideais em $B$ e $B^{(\lambda)}$ coincidem, $\operatorname{logo} B$ também é prima. Além disso,

$$
[x, y]_{\lambda}=(2 \lambda-1)[x, y] .
$$

Ora, A satisfaz $0=[x, y]_{\lambda}^{m}=(2 \lambda-1)^{m}[x, y]^{m}$, portanto $B$ satisfaz $[x, y]^{m}$. Isso significa, tendo em vista os resultados de [Kem80], que $B$ está em uma variedade não matricial de álgebras associativas. Como $B$ também é Nil-semissimples, seguese que $B$ é comutativa, implicando em A comutativa. Voltando ao item anterior, concluímos que $\mathbf{A}$ é associativa e comutativa.

3. $Z^{-1} \mathbf{A}$ é flexível quadrática simples. Neste caso, pelo Lema 5.1.3, $\left(\mathbb{Z}^{-1} A\right)^{(+)} \cong$ $J(V, f)$, em que $J(V, f)$ é uma álgebra de Jordan tipo Clifford (com uma forma bilinear simétrica não degenerada $f$ ). É sabido que, nestas condições, para quaisquer $a, b, c \in Z^{-1} \mathbf{A},(a, b, c)=(a, b, c)_{+}$. Logo, $(a, b, c)_{+}^{m}=0$, um absurdo.

4. $\mathbf{A}^{(+)}$é associativa. Pelo Teorema 5.1.13, sabemos que $\mathbf{A} \subseteq(F(X), *)$ é uma subálgebra. Todavia, $(F(X), *)$ não tem divisores de zero, portanto as identidades satisfeitas por A podem ser rescritas da seguinte forma:

$$
\begin{gathered}
(x, y, z)^{m}=0 \Longrightarrow(x, y, z)=0 ; \\
{[x, y]^{m}=0 \Longrightarrow[x, y]=0 .}
\end{gathered}
$$

Ou seja, A é associativa e comutativa.

Desta forma, provamos que se uma variedade $\mathcal{M}$ de álgebras de Jordan não comutativas satisfaz $(x, y, z)^{m} \equiv 0$ e $[x, y]^{m} \equiv 0$, para certo $m$, então $\mathcal{M}$ é variedade não matricial.

Agora, provemos as demais implicações.

(i) $\Longrightarrow$ (iv): Seja $\mathbf{A} \in \mathcal{M}$ uma álgebra de Jordan não comutativa arbitrária. Consideremos a álgebra $\overline{\mathbf{A}}=A / \operatorname{Nil}(\mathbf{A})$. Pelo Lema 5.3.5, $\mathbf{A}$ é associativa e comutativa e como $\operatorname{Nil}(\overline{\mathbf{A}})=$ 
$(\overline{0})$, já é sabido que $\mathbf{A}$ não contém elementos nilpotentes não triviais, ou seja, se $\bar{x} \in \overline{\mathbf{A}}$ é nilpotente, então $\bar{x}=\overline{0}$. Isso é o mesmo que dizer que $x \in \operatorname{Nil}(\mathbf{A})$.

Seja $y \in \mathbf{A}$ um elemento nilpotente e seja $\bar{y}$ sua imagem através do homomorfismo $\mathbf{A} \rightarrow \overline{\mathbf{A}}$. Dessa forma, $y^{k}=0 \Longrightarrow \bar{y}^{k}=\overline{0}$ e, pela observação feita antes, $y \in \operatorname{Nil}(\mathbf{A})$.

(i) $\Longrightarrow(\mathbf{v})$ : Isso é claro, pois (i) $\Longrightarrow($ iv) e, evidentemente, (iv) $\Longrightarrow(\mathrm{v})$.

(i) $\Longrightarrow(\mathbf{v i})$ : Lembremos que (i) $\Longrightarrow($ iv). Ora, Nil(A) é o ideal maximal de A que contém os ideais à direita, à esquerda e bilaterais, logo o ideal à direita (à esquerda) gerado por $a$ está em $\operatorname{Nil}(\mathbf{A})$.

(i) $\Longrightarrow($ vii): Mesmo argumento de (i) $\Longrightarrow($ vi).

(i) $\Longrightarrow$ (viii): Seja $\mathbf{A} \in \mathcal{M}$ uma álgebra de Jordan não comutativa gerada pelo um conjunto finito de elementos algébricos sobre um corpo $F$. Como A é álgebra de Jordan não comutativa, então A é potências associativas e na subseção 2.3.1 estabelecemos o seu radical algébrico alg $(\mathbf{A})$. Consideremos o radical $F$-algébrico $\mathfrak{a l g}(\mathbf{A})$ da álgebra $\mathbf{A}$. Provemos que $\mathbf{A}=\mathfrak{a l g}(\mathbf{A})$. Pelo Teorema 5.1.2, a álgebra quociente $\overline{\mathbf{A}}=\mathbf{A} / \mathfrak{a l} \mathfrak{g}_{F}(\mathbf{A})$ é isomorfa a um produto subdireto de álgebras de Jordan não comutativas primas $\mathbf{A}_{\alpha}$ com $\mathfrak{a l} \mathfrak{g}_{F}\left(\mathbf{A}_{\alpha}\right)=0$.

Como $\operatorname{Nil}\left(\mathbf{A}_{\alpha}\right) \subseteq \mathfrak{a l g}_{F}\left(\mathbf{A}_{\alpha}\right)=0$, todas as álgebras $\mathbf{A}_{\alpha}$ são Nil-semissimples, logo a álgebra $\overline{\mathbf{A}}$ é Nil-semissimples e portanto associativa e comutativa.

Mas a álgebra $\overline{\mathbf{A}}$ continua ser gerada por um conjunto finito de elementos algébricos, portanto ela tem dimensão finita sobre $F$. Em particular, $\mathfrak{a l g}_{F}(\overline{\mathbf{A}})=\overline{\mathbf{A}}$, donde $\overline{\mathbf{A}}=(\overline{0})$ e $\mathbf{A}=\mathfrak{a l g}_{F}(\mathbf{A})$.

Então a álgebra $\mathbf{A}$ é algébrica sobre $F$, logo a álgebra de Jordan $\mathbf{A}^{(+)}$também é algébrica sobre $F$. Pelo Lema 5.2.11, a álgebra $\mathbf{A}^{(+)}$é gerada por um conjunto finito de elementos algébricos e, por ser não matricial (Proposição 5.3.6), pelo Teorema A, tem dimensão finita sobre $F$. Finalmente, $\operatorname{dim}_{F} \mathbf{A}=\operatorname{dim}_{F} \mathbf{A}^{(+)}<\infty$.

Nos casos anteriores, nós tínhamos equivalência de todas as afirmações (i)-(viii). Agora isso já não é certo: a condição (iv) não implica (i). Com efeito, consideremos a variedade $\mathcal{V}$ de álgebras de Jordan não comutativas, determinada pela identidade $(x, y, z)_{+}=0$ sobre um corpo $F$ de característica zero. Para cada álgebra $A \in \mathcal{V}$, a álgebra $A^{(+)}$é associativa e comutativa, portanto o radical $\mathrm{Nil} A^{(+)}$coincide com o conjunto de elementos nilpotentes em $A^{(+)}$. É bem conhecido que em característica zero $\mathrm{Nil} A=\mathrm{Nil} A^{(+)}$[Sch94], portanto $A$ satisfaz a condição (iv). Mas a variedade $\mathcal{V}$ contem a álgebra $(F[X], *)$ do exemplo 5.1.5, que evidentemente não satifaz i). Portanto, $(i v) \nRightarrow(i)$.

Nossa intenção inicial era a de estabelecer as equivalências (i) - (viii), como feito para as classes de álgebras estudadas anteriormente. Para a definição por nós adotada (Definição 10), não é possível estabelecer tais equivalências, mas é possível estabelecer algumas identidades (veja 5.10) não triviais em variedades não matriciais de álgebras de Jordan não comutativas.

No caso não matricial para álgebras de Jordan não comutativas, estabelecemos que álgebras primas Nil-semissimples são associativas e comutativas. Nosso principal empecilho, em relação às técnicas usada nos casos de Jordan e alternativas, foi o caso da álgebra $(F[X], *)$ definida a partir dos colchetes de Poisson (Exemplo 5.1.5). 
Um problema a ser formulado em virtude deste "caso patológico" pode ser dado da seguinte forma:

Questão 5.3.1. Quais identidades definem a variedade gerada por $(F[X], *)$ ?

Um outra questão é avaliar se álgebras de Jordan não comutativas simples são corpos numa variedade não matricial (de álgebras de Jordan não comutativas), como definido em 10.

Neste capítulo pudemos notar que a generalização de um resultado não matricial para álgebras de Jordan não comutativas não é trivial. Particularmente, não oferecemos uma resposta à classe das álgebras de Jordan não comutativas, mas nos restringimos à classe das álgebras de Jordan não comutativas admissíveis. Todavia, atendeu o nosso programa inicial de estabelecer uma condição não matricial para uma variedade "larga" o suficiente de forma conter as álgebras associativas e comutativas (veja o final do Capítulo 4). De fato, como visto nos exemplos 5.2.9 e 5.2.7, as álgebras associativas e as álgebras comutativas são admissíveis. O que não é certo é que tais variedades são essenciais. Se A é álgebra comutativa, obviamente $\mathbf{A}=\mathbf{A}^{(+)}$. Por outro lado, se $\mathbf{A}$ é associativa, $\mathbf{A}^{(+)}$é sempre de Jordan. Em ambos os casos, $\mathbf{A}^{(+)}$não é, necessariamente, uma PI-álgebra de Jordan. A condição de "ser essencial" induz identidades não triviais numa variedade $\mathcal{V} \subseteq$ NCJ. 


\section{Capítulo 6}

\section{Conclusões}

Neste trabalho, apresentamos algumas caracterizações em variedades não matriciais para álgebras não associativas. A literatura atual já dispõe de muitos resultados acerca de variedades não matriciais de álgebras associativas, todavia não há registro de um caso não matricial para álgebras não associativas.

A originalidade deste trabalho consiste em dois motes principais:

- Alterar a forma de caracterizar variedades não matriciais.

- Generalizar técnicas de não matricialidade para álgebras não associativas.

Até o presente momento, variedades não matriciais eram pensadas como "retirar um tipo matricial de álgebra" de forma a caracterizar a variedade. Nossa paradigma é o de caracterizar a não matricialidade de uma variedade através de algumas condições acerca das álgebras simples contidas nela ou estabelecer condições acerca de álgebras $\mathcal{R}$ semissimples, para algum radical $\mathcal{R}$ conhecido nessa variedade (por exemplo, impusemos as condições de associatividade e comutatividade para as primas Nil-semissimples, no estudo de caso para as álgebras de Jordan não comutativas).

Consideramos que é vantajosa esta forma de definir uma variedade não matricial, pois encontrar um "candidato matricial" adequado (para caracterizar uma variedade não matricial) pode se tornar uma tarefa pouco trivial, talvez até impossível.

Note-se também que as técnicas aqui usadas diferem do caso associativo, que é conhecido na literatura. Identidades não associativas dificultam um pouco mais as operações multiplicativas, de sorte que foi necessário uma pesquisa mais ampla e criteriosa para os casos não associativos aqui considerados.

O cerne desta pesquisa, no entanto, continuou sendo a mesma para o caso associativo: avaliar radicais que nos interessam e qual a estrutura determinada por tais radicais. Além disso, se constituiu um problema fundamental descobrir quais identidades estavam relacionadas aos casos não matriciais avaliados.

\subsection{Sugestões para Pesquisas Futuras}

Considere o Teorema 2.5.2 já enunciado no primeiro capítulo, devido a Kemer:

"Se $\mathcal{M}$ é uma variedade não matricial e $G \otimes G \notin \mathcal{M}$, em que $G$ é a álgebra de Grassmann, então $\mathcal{M}$ satisfaz a seguinte identidade, para algum natural $r$ :

$$
\left[x_{1}, y_{1}, z_{1}\right] \cdot \ldots \cdot\left[x_{r}, y_{r}, z_{r}\right]=0 " .
$$


Neste trabalho (c. [Kem80]), Kemer usa técnicas de superálgebras (embora não use a nomenclatura "superálgebra", as técnicas são muito similares) para obtenção destes resultados. Todavia, há uma vantagem aí: ele se utiliza do fato de que álgebras de Grassmann são associativas para falar de variedades não matriciais de álgebras associativas.

Deixaremos às futuras pesquisas em álgebra as seguintes questões:

Questão 11. Qual o análogo do Teorema 2.5.2 em estruturas não associativas?

Sendo mais específico para os casos que nós estudamos:

Questão 12. Qual o análogo da álgebra de Grassmann para álgebras de Jordan?

Questão 13. Qual o análogo da álgebra de Grassmann para álgebras alternativas?

Questão 14. Qual o análogo da álgebra de Grassmann para álgebras de Jordan não comutativas?

Uma outra proposta para definir variedades não matriciais surgiu durante a pesquisa: "diremos que uma variedade $\mathcal{V}$, em uma certa classe de álgebras, é não matricial finita se qualquer álgebra simples de dimensão finita é um corpo". Claramente, de acordo com a Definição 5, variedades não matriciais são variedades não matriciais finitas.

Problema 15. Seja $\mathcal{U} \in\{$ Assoc, Jord, Alt $\}$. Determinar quais variedades não matriciais finitas em $\mathcal{U}$ também são não matriciais (de acordo com a Definição 5).

No capítulo que tratou das álgebras de Jordan não comutativas, expusemos as seguintes condições:

(NM1) Qualquer álgebra prima Nil-semissimples em $\mathcal{V}$ é comutativa e associativa.

(NM2) Qualquer álgebra simples em $\mathcal{V}$ é corpo.

E voltamos às questões a elas associada:

Questão 16. Na variedade NCJ das álgebras de Jordan não comutativas, se $\mathcal{V} \subseteq$ NCJ é variedade satisfazendo $(N M 2)$, então $\mathcal{V}$ satisfaz $(N M 1)$ ?

Seja A a álgebra do Exemplo 5.3.1 e seja $\operatorname{var}(\mathbf{A})$ a variedade por ela gerada.

Questão 17. Álgebras simples em $\operatorname{var}(\mathbf{A})$ são corpos?

Problema 18. Determinar as identidades que definem a variedade $\operatorname{var}(\mathbf{A})$ da questão anterior.

Conforme mencionado no final capítulo sobre álgebras de Jordan não comutativas, oferecemos uma caracterização não matricial para uma classe específica de álgebras de Jordan não comutativas: a classe das álgebras de Jordan não comutativas admissíveis. Deixamos o seguinte problema:

Problema 19. Apresentar uma caracterização não matricial em toda a variedade NCJ das álgebras de Jordan não comutativas. 


\subsection{Considerações Finais}

Este trabalho propõe-se a ser uma pequena contribuição à comunidade matemática. Esperamos que, futuramente, os resultados aqui obtidos ou as técnicas aqui desenvolvidas ajudem futuros pesquisadores na solução de problemas.

Além disso, nossa expectativa é a mudança paradigmática com respeito à definição de "não matricialidade" na Álgebra. Certamente, os autores continuarão a investigar e aperfeiçoar os resultados alcançados.

Agradecemos ao CNPq e à FAPESP pelo apoio à pesquisa concedido particularmente ao candidato e ao orientador, nesta tese. 
78 CONCLUSÕES 


\section{Referências Bibliográficas}

[Alb48] ALBERT, A. A. Power-associative rings. Transactions of the American Mathematical Society, 64, p. 552-593, 1948. 57, 59

[Am52] AMITSUR, S. A. A general Theory of Radicals I. American Journal of Mathematics, 74, p. $774-786,1952$.

[Am54a] AMITSUR, S. A. A general Theory of Radicals II. Am. J. Math., 76, p. 100 $125,1954$.

[Am54b] AMITSUR, S. A. A general Theory of Radicals III. Am. J. Math., 76, p. $126-136,1954$.

[BRT97] BILliG, Y., RILEY, D., TASIC, V. Nonmatrix Varieties and Nil-Generated Algebras Whose Units Satisfy a Group Identity. Journal of Algebra, 190, p. 241-252, 1997. 24

[Cek79] CEKANU, G. P. Distinguished varieties of associative algebras. Math. Issled Vyp., 49, p. 149 - 158, 1979. iii, v, 25, 44, 51

[Div64] DIVINSKY, N. J. Rings and Radicals. University of Toronto, 1964. (Mathematical Expositions) 12

[GW04] GARDNER, B. J.; WIEGANDT, R. Radical Theory of Rings. Marcel Deker, 2004. (Pure And Applied Mathematics, 261) 12, 15

[G69] GRAY, M. A Radical Approach to Algebra. Addison-Wesley, 1969. 3

[Hen69] HENTZEL, I. R. (-1,1) Rings. Proceedings of the American Mathematical Society, 22, n. 2, p. $367-374,1969.52$

[Hen70] HentzeL, I. R. (-1,1) Algebras. Proc. Amer. Math. Soc., 24, n. 1, p. 24-28, 1970.

[Hen72] HENTZEL, I. R. Nil semisimple (-1,1) rings. J. Algebra, 30, p. 236-258, 1972. 52

[Hung74] HUNGERFORD, T. W. Algebra. Springer, 1974. (Graduate Texts in Mathematics, 73) 3

[Jec03] JECH, Thomas J. Set Theory: The Third Millennium Edition, Revised and Expanded. New York: Springer, 2003. (Springer Monographs in Mathematics) 12

[Jac43] JACOBSON, N. The Theory of Rings. AMS, Mathematical Surveys II, 1943. 
[Jac56] JACOBSON, N. Structure of Rings. Am. Math. Soc. Coll. Publ., 76, 1956.

[Jac69] JACOBSON, N. Structure and Representations of Jordan Algebras. Am. Math. Soc. Coll. Publ., 39, 1969.

[Kem80] KEMER, A. R. Nonmatrix varieties. Algebra and Logic, 19, vol. 3, p. 157 - 178, 1980. $25,40,72,76$

[Kok58] KOKORIS, L. A. Simple nodal noncommutative Jordan algebras. Proc. Am. Math. Soc., 9, p. 652,-654, 1958. 57, 59

[Kur53] KUROSH, A. Radicals of rings and algebras. Math. Sbornik, 33, p. 13-26, 1953.

[Lang02] LANG, S. Algebra. Springer, 2002. 3

[Lat77] LATYSHEV, V. N. The complexity of non-matrix varieties of associative algebras. I, II. Algebra i Logika 16, 2, p. 149 - 183, 184 - 199, 1977. iii, v, 1, 24

[McCr04] MCCRIMMON, K. A Taste of Jordan Algebras. Springer, 2004. (Universitext) $12,30,35$

[MPR2011] MISHCHENKO, S. P., PETROGRADSKY V. M., REGEV A. Characterization of non-matrix varieties of associative algebras. Israel Journal of Mathematics, 182, p. 337-348, 2011. iii, v, 25, 41, 44, 49

[Med88-1] MEDVEDEV Y. A. Nilradicals of finitely generated Jordan PI-algebras. Sib. Math. J., No. 1. 29, p. 84-94, 1988.

[Med88-2] MEDVEDEV Y. A. Representations of finitely generated Jordan PI-algebras. Izv. Akad. Nauk SSSR Ser. Mat., Issue 1. 52, p. 64-78, 1988. 35, 41

[Pch75] PCHELINTSEV, S. V. Nilpotency of the associator ideal of a free finitely generated $(-1,1)$ ring. Algebra and Logic. 14 v. 5, p. 334-353, 2004. 52

[Pch04] PCHELINTSEV, S. V. Nilpotency of the Alternator Ideal of a Finitely Generated Binary (-1,1)-Algebra. Siberian Mathematical Journal. 45 n. 2, p. 356 - 375, 2004. 52

[Sch55] SCHAFER, R. D. Noncommutative Jordan algebras of characteristic 0. Proc. Amer. Math. Soc. 6, p. $472-475,1955.57$

[Sch94] SCHAFER, R. D. An Introduction to Nonassociative Algebras. Dover, 1994. 58, 73

[Sm71] SMITH, V. G. Noncommutative Jordan algebras of capacity two. Trans. Amer. Math. Soc. v. 1 158, p. 151 - 159, 1971. 57, 58

[Sk89] SKOSYRSKII V. G. Strictly prime noncommutative Jordan algebras. Proc. of Inst. Math. Siberian Branch of the USSR Academy of Sciences. 16, p. 131-163, 1989. 57,60

[Sk91] SKOSYRSKII, V. G. Noncommutative Jordan algebras a under the condition that $\mathbf{A}^{(+)}$is associative. Siberian Mathematical Journal. Vol. 32 6, p. 1024-1030, 1991. 59, 61 
[Sh71] SHESTAKOV, I. P. Certain classes of noncommutative Jordan rings. Algebra and Logic, Vol. 10. 4, p. 252-280, 1971. 57, 59, 62, 65, 66

[Sh83] SHESTAKOV, I. P. Finitely generated special Jordan and alternative PIalgebras. Sib. Math., Vol. 1. 22, p. 31-40, 1983. 48

[ZSh73] ZHEVLAKOV, K. A., SHESTAKOV I. P. On local finiteness in the sense of Shirshov. Algebra i Logika (English: Algebra and Logic), Eng. Transl. 12, p. 23 -41, 1973. 17, 18, 19, 20, 21, 42, 47, 51, 61

[Zel78] ZEL'MANOV, E. I. On Prime Jordan Algebras. Algebra i Logika (English: Algebra and Logic), Eng. Transl. 18, p. $162-175,1979.35$

[Zel79] ZEL'MANOV, E. I. Jordan Division Algebras. Algebra i Logika (English: Algebra and Logic), Eng. Transl. 18, p. $286-310,1979.35$

[Zel83] ZEL'MANOV, E. I. On Prime Jordan Algebras II. Siberian Mathematical Journal, Eng. Transl. 24, p. 89 - 104, 1983. 35

[ZSSS82] ZHEVLAKOV, K. A., SLIN'KO, A. M., SHESTAKOV, I. P., SHIRSHOV, A. I. Rings That Are Nearly Associative. Academic Press, 1982. (Pure and Applied Mathematics) $3,6,7,11,12,23,30,32,34,35,45,46,48,49,50,58,63$ 